\title{
A review of the foot function index and the foot function index - revised
}

Elly Budiman-Mak ${ }^{1,2^{*}}$, Kendon J Conrad ${ }^{3}$, Jessica Mazza ${ }^{4}$ and Rodney M Stuck ${ }^{5,6}$

\begin{abstract}
Background: The Foot Function Index (FFI) is a self-report, foot-specific instrument measuring pain and disability and has been widely used to measure foot health for over twenty years. A revised FFI (FFI-R) was developed in response to criticism of the FFI. The purpose of this review was to assess the uses of FFI and FFI-R as were reported in medical and surgical literature and address the suggestions found in the literature to improve the metrics of FFI-R.

Methods: A systematic literature search of PubMed/Medline and Embase databases from October 1991 through December 2010 comprised the main sources of literature. To enrich the bibliography, the search was extended to BioMedLib and Scopus search engines and manual search methods. Search terms included FFI, FFI scores, FFI-R. Requirements included abstracts/full length articles, English-language publications, and articles containing the term "foot complaints/problems." Articles selected were scrutinized; EBM abstracted data from literature and collected into tables designed for this review. EBM analyzed tables, KJC, JM, RMS reviewed and confirmed table contents. KJC and $J M$ reanalyzed the original database of FFI-R to improve metrics.

Results: Seventy-eight articles qualified for this review, abstracts were compiled into 12 tables. FFI and FFI-R were used in studies of foot and ankle disorders in 4700 people worldwide. FFI Full scale or the Subscales and FFI-R were used as outcome measures in various studies; new instruments were developed based on FFI subscales. FFI Full scale was adapted/translated into other cultures. FFI and FFI-R psychometric properties are reported in this review. Reanalysis of FFI-R subscales' confirmed unidimensionality, and the FFI-R questionnaires' response categories were edited into four responses for ease of use.
\end{abstract}

Conclusion: This review was limited to articles published in English in the past twenty years. FFI is used extensively worldwide; this instrument pioneered a quantifiable measure of foot health, and thus has shifted the paradigm of outcome measure to subjective, patient-centered, valid, reliable and responsive hard data endpoints. Edited FFI-R into four response categories will enhance its user friendliness for measuring foot health.

Keywords: FFI, FFI-R, FFI adaptation/translation, FFI scores, Foot health measures

\section{Background}

Foot problems commonly arise during our daily living activities [1,2]. The prevalence of foot problems in general ranges between $10 \%$ and $24 \%$ [3]. Their prevalence is higher among older individuals and in chronic rheumatoid arthritis (RA), gout, and diabetes mellitus with peripheral neuropathy [4]. Foot pain and disability can affect workers' productivity, work absenteeism, and

\footnotetext{
*Correspondence: Elly.Mak@va.gov

'Center for Management of Complex Chronic Care, Staff Physician, Medical Service, Hines, VA Hospital, 5000 South 5th Ave, Hines, IL 60141-3030, USA ${ }^{2}$ Department of Medicine Loyola University Stritch School of Medicine, Loyola University of Chicago, Maywood, IL 60513, USA

Full list of author information is available at the end of the article
}

other issues [5,6]. Because pain and disability are subjective complaints, they are difficult to quantify without a valid patient report of the degree to which an individual is experiencing foot pain. Without a valid measure, problems arise in documenting foot health status, tracking the progression of diseases, and establishing the efficacy of treatment, including assessment of treatment satisfaction and of health related quality of life from a personal perspective.

In 1991, the Foot Function Index (FFI) was developed as a self-reporting measure that assesses multiple dimensions of foot function on the basis of patient-centered values. The FFI consists of 23 items divided into 3 subscales that 
quantify the impact of foot pathology on pain, disability, and activity limitation in patients with RA [7]. The FFI was developed using the classical test theory (CTT) [8] method. It has been found to have good reliability and validity and has had wide appeal to clinicians and research scientists alike $[3,9,10]$. In the past 20 years, the FFI has been widely used by clinicians and investigators to measure pain and disability in various foot and ankle disorders and its use has expanded to involve children, adults, and older individuals. Furthermore, the FFI has been widely used in the study of various pathologies and treatments pertaining to foot and ankle problems such as congenital, acute and chronic diseases, injuries, and surgical corrections.

In 2006, the FFI was revised (the FFI-R) on the basis of criticisms from researchers and clinicians; items were added, including a scale to measure psychosocial activities and quality of life related to foot health [11].

A literature review was conducted to develop a theoretical model of foot functioning [12], based on the World Health Organization International Classification of Functioning (ICF) model. The FFI-R items were developed from the original 23 FFI items, and more items were added as a result of the literature review. As a result of clinicians and patients' input, the final draft of the FFI-R, which consisted of 4 subscales and 68 items, was completed. The results were the FFI-R long form (FFI-R L; 4 subscales and 68 items) and the FFI-R short form (FFI-R S; 34 items) as total foot function assessment instruments. Both the 68-item and 34-item measures demonstrated good psychometric properties.

The FFI-R in its current form is one of the most comprehensive instruments available. However, in a review article [13], questions were raised about the unidimensionality and independence of FFI-R subscales, and we did not include such reports in our previous article about the FFI-R [11]. We carefully reviewed the comments about the FFI-R and assessed the unidimensionality of the subscales by use of the Rasch model. On the basis of these critiques, the FFI-R required a periodic revision of its metrics to ensure it represented patient-centered health values and state-of-the-art methodology.
Our aim is to assess the contribution of the FFI and FFI-R to the measurement of foot health in the fields of rheumatology, podiatry, and orthopedic medicine. This assessment should enable us to reflect on and improve the quality of the measure. Therefore, we conducted a systematic review of literature pertaining to the FFI and FFI-R that has been published in the English language from October 1991 through December 2010. The objectives were to: (i), Assess the prevalence of uses of the FFI and FFI-R in clinical studies of foot and ankle disorders; (ii), Describe the utility and clinimetric properties of the FFI and FFI-R as they have been applied in various clinical and research settings; (iii), Enumerate the strengths and weaknesses of the FFI and FFI-R as reported in the literature; (iv), Address the suggestions found in the literature for improving the FFI-R metrics.

\section{Methods for systematic search of the literature}

This study was about a systematic review of articles in which the FFI and/or FFI-R were used as measures of a variety of foot and ankle problems. Relevant studies were identified by English language publication searches of the electronic bibliographic databases Pub Med/MEDLINE, EMBASE, BioMedLib and Scopus from October 1991 through December 2010.

\section{Search terms and eligibility criteria}

The key words: foot function index, FFI scores, foot function index scores, and foot function index revised (FFI-R). were used as search terms and was applied to all databases. FFI instruments/measure and/or FFI-R instruments/measure had to be mentioned in the abstracts and in the full articles to be collected for in-depth scrutiny. Articles fulfilling the inclusion criteria were selected for the review. The article criteria included: (i) the words foot function index/FFI or revised foot function index/FFI-R in its reports/measures; (ii) full-length articles; (iii) written in English and published from October 1991 through December 2010; (iv) the study population described needed to have foot complaint(s)/problems; and (v) regardless of the country conducting the study, the full-length article

Table 1 Study type, sample size and sample characteristics

\begin{tabular}{llllll}
\hline Study type & Number & Sample size (N) & N Male & N Female & Age (SD) \\
\hline Measurement & 17 & 1236 & 458 & 763 & $54.9(6.4)$ \\
Surgery & 30 & 1512 & 648 & 857 & $45.1(15.7)$ \\
Orthoses & 19 & 1101 & 493 & 521 & $43.0(15)$ \\
Other intervention & 4 & 170 & 55 & 115 & $47.6(6.1)$ \\
Observational & 8 & 695 & 260 & 432 & $52.2(27.9)$ \\
Total & 78 & 4714 & $1914^{*}(41 \%)$ & $2688^{*}(57 \%)$ & $48.58(4.9)$
\end{tabular}

*Gender not reported in 3 studies: Slattery, M [82] (2001), Clark, H [85] (2010) and Kulig, K [88] (2009). 
must have been published in English or in a foreign language with the abstract in English.

\section{Objectives with method of data collection and organization of tables}

Selected articles that fulfilled the criteria were independently reviewed and collected by the authors to address the objectives and organize collected data into several tables.

\section{Objective 1. Uses of the FFI and FFI-R}

We created four tables to address the first objective of describing the measurement's uses (Tables 1, 2, 3, and 4).

\section{Objective 2. Utility and clinimetric properties}

We designed a data-collection form to address the second objective. This form was assessed in a pilot study by collecting data from ten articles out of the collection of qualified articles; it was revised before being used in its current format. The variables used in this datacollection form were: (i) the instrument and year the article was published; (ii) the first author's name; (iii) the objectives of the study; (iv) the population characteristics, sample size, and diagnosis; (v) psychometric analysis (reliability and validity, etc.); (vi) items/domains/subscales of the FFI or FFI-R used in the study; (vii) response type; and, (viii) a short summary evaluation of each study. Therefore, this data form recorded the analytic statements extracted from each article, and 6 tables were created (Tables 5, 6, 7, 8, 9, and 10). Data were arranged in each table in chronological order.

Objective 3. Enumerate the strengths and weaknesses of the FFI and FFI-R as reported in the literature

This was a qualitative summary of the results as found in Table 5 and Table 6.

\section{Objective 4. Improving the FFI-R metrics}

Table 11 summarizes results of the Rasch analysis. This was a reanalysis of the FFI-R database collected in 2002 with the aim of improving FFI-R metrics.

\section{Descriptive analysis methods}

Quantitative data were reported using simple statistics expressed as the sum, means, and standard deviations for continuous variables and as frequencies for categorical data. (Tables 1, 2, 3, and 4) Analytic statements and evaluations/comments for each article collected are summarized in Table 12. This depicts the summary of

Table 2 FFI uses across studies in foot and ankle disorders including diagnoses

\begin{tabular}{|c|c|c|c|c|c|c|}
\hline Diagnosis & Measure & Surgery & Orthosis & Observational & Other & Total \\
\hline Rheumatoid arthritis & 6 & 5 & 7 & 3 & & 21 \\
\hline Osteoarthritis & 2 & 1 & & 1 & & 4 \\
\hline Juvenile arthritis & & & 1 & & & 1 \\
\hline Hallux valgus & 2 & 2 & 1 & & & 5 \\
\hline Hallux rigidus & & 3 & & & & 3 \\
\hline Plantar fasciitis/heel pain & 2 & 2 & 4 & & 3 & 11 \\
\hline Metatarso phalangeal arthritis & & 2 & 2 & & & 4 \\
\hline Chronic foot pain & 3 & 2 & & 1 & & 6 \\
\hline Foot and ankle fracture & 1 & 5 & $1^{* *}$ & 1 & & 8 \\
\hline Posterior tibial tendon pain & & & 1 & & 1 & 2 \\
\hline Bone graft & & 1 & & & & 1 \\
\hline Ankle deformity & & 2 & & & & 2 \\
\hline Flat foot & & 1 & & & & 1 \\
\hline Cavovarus Charcot-Marie-Tooth & & 2 & & & & 2 \\
\hline Osteo-chondral lesion of talus-tibia & & 1 & & & & 1 \\
\hline Failed total ankle arthrodesis & & 1 & & & & 1 \\
\hline Club foot & & 1 & & & & 1 \\
\hline Diabetic neuropathy & & & & 1 & & 1 \\
\hline Mid foot pain & 1 & & 2 & & & 3 \\
\hline Paget disease & & & & 1 & & 1 \\
\hline Total & 17 & $31^{*}$ & 19 & 8 & 4 & $79^{*}$ \\
\hline
\end{tabular}

*Two different diagnoses occurred in one study, **Hemophilic ankle arthropathy. 
Table 3 FFI Uses across studies conducted internationally

\begin{tabular}{|c|c|c|c|c|c|c|}
\hline Country & Measure & Surgery & Orthosis & Observational & Other & Total \\
\hline Australia & 2 & 1 & 1 & & & 4 \\
\hline Austria & & 2 & & & & 2 \\
\hline Brazil & & & 2 & & & 2 \\
\hline Canada & & 2 & & 1 & & 3 \\
\hline Czech Rep. & & 2 & & & & 2 \\
\hline France & & 1 & & & & 1 \\
\hline Germany & 1 & 1 & & 2 & 1 & 5 \\
\hline Japan & & 1 & & & & 1 \\
\hline So. Korea & & & 1 & & & 1 \\
\hline Netherlands & 2 & 7 & & & & 9 \\
\hline New Zealand & & & 1 & & & 1 \\
\hline Slovenia & & & 1 & 1 & & 2 \\
\hline Sweden & & 1 & & & & 1 \\
\hline Taiwan & 1 & & & & & 1 \\
\hline Turkey & 1 & & & 2 & & 3 \\
\hline UK & 2 & 1 & 3 & 2 & & 8 \\
\hline USA & 8 & 12 & 9 & & 3 & 32 \\
\hline Total & 17 & 31 & 18 & 8 & 4 & 78 \\
\hline
\end{tabular}

FFI and FFI-R uses as illustrated in Objective 2, and in six tables (Tables 5, 6, 7, 8, 9 and 10).

\section{Rasch analysis method}

To address specific critiques of the FFI-R found in the literature, the unidimensionality of the FFI- $R$ and its subscales were evaluated against the Rasch model. The statistical package Winsteps version 3.72.3 [14] was used to conduct a principal components analysis (PCA) of the standardized residuals to determine whether substantial subdimensions existed within the items [15-17] and whether the FFI-R L, the FFI-R S, and the 5 subscales were unidimensional. The criterion used to define unidimensionality was a large variance (> 40\%) explained by the measurement dimension [18]. Unexplained variance in the first contrast of the data should be small and fall under the criterion of $15 \%$ for a rival factor. We chose a ratio of variance of at least 3 to 1 in the first principal component [19], compared to the variance of the first component of residuals.

\section{Rasch reliability statistics}

Reliability was estimated with Cronbach's Alpha and Rasch person reliability statistics. Both indices reflect the proportion of variance of the person scores or measures to total variance (i.e., including measurement error). Unlike Cronbach's Alpha, Rasch person reliability is based on the estimated locations of persons along the measurement continuum, excluding those with measures reflecting extreme (zero or perfect) scores and including cases with missing data. For both indices, our criterion for acceptability was .80 .

Table 4 FFI Full scale and subscale used across studies

\begin{tabular}{|c|c|c|c|c|c|c|}
\hline $\mathrm{FFI}$ & Measure & Surgery & Orthosis & Observational & Other & Total \\
\hline FFI Full scale (3 domains) & 10 & 21 & 14 & 6 & & 51 \\
\hline FFI Pain scale & 2 & 1 & 2 & 2 & 3 & 10 \\
\hline FFI Disability scale & & 1 & & & & 1 \\
\hline FFI Pain and Disability scale & 3 & 3 & 1 & & 1 & 8 \\
\hline FFI - 5pts & 1 & 4 & & & & 5 \\
\hline FFI-R Long form & 1 & & 2 & & & 3 \\
\hline FFI Used in studies & 17 & 30 & 19 & 8 & 4 & 78 \\
\hline
\end{tabular}


Table 5 Studies of foot function measures

\begin{tabular}{|c|c|c|c|c|c|c|c|}
\hline Instrument & $1^{\text {st }}$ Author & Objective & $\begin{array}{l}\text { Population } \\
\text { (N, Sex, Age, Dx, } \\
\text { location) }\end{array}$ & $\begin{array}{l}\text { Psychometric } \\
\text { analysis }\end{array}$ & $\begin{array}{l}\text { Items/domains/ } \\
\text { subscales/item } \\
\text { sources }\end{array}$ & $\begin{array}{l}\text { Response } \\
\text { type }\end{array}$ & Summary evaluation \\
\hline \multirow[t]{5}{*}{$\begin{array}{l}\text { Foot Function Index, } \\
1991\end{array}$} & \multirow[t]{5}{*}{$\begin{array}{l}\text { Budiman-Mak, } \\
\text { E [7] }\end{array}$} & \multirow[t]{5}{*}{$\begin{array}{l}\text { Instrument } \\
\text { Development }\end{array}$} & N: 87 (78 male) & \multirow[t]{5}{*}{$\begin{array}{l}\text { Classical Test } \\
\text { Theory }\end{array}$} & 23 items & \multirow[t]{5}{*}{$\begin{array}{l}\text { Visual } \\
\text { Analog } \\
\text { Scale }\end{array}$} & $\begin{array}{l}\text { Good clinimetrics, } \\
\text { applicable to various } \\
\text { age groups and } \\
\text { varieties of foot and } \\
\text { ankle pathologies. }\end{array}$ \\
\hline & & & Mean age: 61 & & 3 domains & & \multirow[t]{4}{*}{ Conclusion: Positive } \\
\hline & & & (Range: 24-79) & & $\begin{array}{l}\text { Pain, difficulty } \\
\text { and activity } \\
\text { limitation } \\
\text { subscales } \\
\text { clinician }\end{array}$ & & \\
\hline & & & Dx: RA foot & & & & \\
\hline & & & Location: USA & & & & \\
\hline \multirow[t]{4}{*}{$\begin{array}{l}\text { Foot Function Index } \\
\text { Pain (left/right), } 1996\end{array}$} & \multirow[t]{4}{*}{ Saag, KG [23] } & \multirow[t]{4}{*}{$\begin{array}{l}\text { Foot Function } \\
\text { Index pain } \\
\text { scale; Compare } \\
\text { right/left foot }\end{array}$} & N: 63 (13 male) & \multirow[t]{4}{*}{$\begin{array}{l}\text { Classical Test } \\
\text { Theory }\end{array}$} & 9 items & \multirow[t]{4}{*}{$\begin{array}{l}\text { Visual } \\
\text { Analog } \\
\text { Scale }\end{array}$} & $\begin{array}{l}\text { This measure of right } \\
\text { vs. left side of the } \\
\text { foot showed good } \\
\text { clinimetric properties }\end{array}$ \\
\hline & & & $\begin{array}{l}\text { Mean age: } 57.5 \\
(S D=11.6)\end{array}$ & & FFI pain subscale & & Conclusion: Positive. \\
\hline & & & Dx: RA & & clinician & & \\
\hline & & & Location: USA & & & & \\
\hline \multirow{4}{*}{$\begin{array}{l}\text { Foot Function Index/ } \\
\text { Foot Health Status } \\
\text { Questionnaires } \\
\text { (FHSQ), } 1998\end{array}$} & \multirow[t]{4}{*}{ Bennet PJ [9] } & \multirow{4}{*}{$\begin{array}{l}\text { Development } \\
\text { of FHSQ, a } \\
\text { new measure }\end{array}$} & N: 111 (25 male) & \multirow[t]{4}{*}{$\begin{array}{l}\text { Classical Test } \\
\text { Theory }\end{array}$} & 13 items & \multirow[t]{4}{*}{ Likert } & $\begin{array}{l}\text { FHSQ has good } \\
\text { clinimetrics. }\end{array}$ \\
\hline & & & $\begin{array}{l}\text { Mean age: } 54 \\
(S D=20)\end{array}$ & & $\begin{array}{l}4 \text { domains } \\
\text { clinician }\end{array}$ & & Conclusion: Positive. \\
\hline & & & $\begin{array}{l}\text { Dx: Osteoarthritis } \\
\text { hallux valgus }\end{array}$ & & & & \\
\hline & & & Location: Australia & & & & \\
\hline \multirow{4}{*}{$\begin{array}{l}\text { Foot Function Index/ } \\
\text { Ankle Osteoartitis } \\
\text { Score (AOS), } 1998\end{array}$} & \multirow[t]{4}{*}{$\begin{array}{l}\text { Domsic, RT } \\
\text { [24] }\end{array}$} & \multirow{4}{*}{$\begin{array}{l}\text { AOS consisted } \\
\text { of Foot } \\
\text { Function Index } \\
\text { pain and } \\
\text { disability scales }\end{array}$} & $\mathrm{N}: 36$ (12 male) & \multirow[t]{4}{*}{$\begin{array}{l}\text { Classical Test } \\
\text { Theory }\end{array}$} & 18 items & \multirow{4}{*}{$\begin{array}{l}\text { Visual } \\
\text { Analog } \\
\text { Scale }\end{array}$} & $\begin{array}{l}\text { AOS had good } \\
\text { clinimetrics. }\end{array}$ \\
\hline & & & $\begin{array}{l}\text { Mean age: } 52.7 \\
\text { (Range: 16-79) }\end{array}$ & & $\begin{array}{l}2 \text { Domains } \\
\text { clinician }\end{array}$ & & Conclusion: Positive. \\
\hline & & & $\begin{array}{l}\text { Dx: Ankle osteo- } \\
\text { arthritis }\end{array}$ & & & & \\
\hline & & & Location: USA & & & & \\
\hline \multirow[t]{4}{*}{$\begin{array}{l}\text { Foot Function Index/ } \\
\text { Foot Function Index- } \\
5 \text { pts in Dutch, } 2002\end{array}$} & \multirow[t]{4}{*}{$\begin{array}{l}\text { Kuyvenhoven, } \\
\text { MM [3] }\end{array}$} & \multirow[t]{4}{*}{$\begin{array}{l}\text { Foot Function } \\
\text { Index in Dutch }\end{array}$} & N: 206 (78 male) & \multirow[t]{4}{*}{$\begin{array}{l}\text { Classical Test } \\
\text { Theory }\end{array}$} & 15 items & \multirow[t]{4}{*}{$\begin{array}{l}\text { 5-point } \\
\text { Likert }\end{array}$} & $\begin{array}{l}\text { Adaptation of Foot } \\
\text { Function Index to } 5 \\
\text { point Likert, used as a } \\
\text { generic measure in } \\
\text { foot and ankle } \\
\text { problems. }\end{array}$ \\
\hline & & & $\begin{array}{l}\text { Mean age: } 61 \\
(\mathrm{SD}=10)\end{array}$ & & $\begin{array}{l}2 \text { domains: pain } \\
\& \text { disability } \\
\text { clinician }\end{array}$ & & Conclusion: Positive. \\
\hline & & & $\begin{array}{l}\text { Dx: OA with } \\
\text { limited mobility } \\
\text { and pain }\end{array}$ & & & & \\
\hline & & & $\begin{array}{l}\text { Location: } \\
\text { Netherlands }\end{array}$ & & & & \\
\hline $\begin{array}{l}\text { Foot Function Index/ } \\
\text { Foot Health Status } \\
\text { Questionnaire (FHSQ), } \\
2002\end{array}$ & $\begin{array}{l}\text { Landorf, KB } \\
{[10]}\end{array}$ & $\begin{array}{l}\text { Validation of } \\
\text { FHSQ to Foot } \\
\text { Function Index }\end{array}$ & N: 17 (4 male) & $\begin{array}{l}\text { Non- } \\
\text { parametric } \\
\text { statistics }\end{array}$ & FHSQ & $\begin{array}{l}\text { 5-point } \\
\text { Likert }\end{array}$ & $\begin{array}{l}\text { FHSQ has less items } \\
\text { than FFI and was } \\
\text { printed in larger font } \\
\text { for ease of use. }\end{array}$ \\
\hline
\end{tabular}


Table 5 Studies of foot function measures (Continued)

\begin{tabular}{|c|c|c|c|c|c|c|c|}
\hline & & & $\begin{array}{l}\text { Mean age: } 44.6 \\
(S D=10.5) \text { (Range } \\
24-72)\end{array}$ & & 13 items & & Conclusion: Positive. \\
\hline & & & $\begin{array}{l}\text { Dx: Painful plantar } \\
\text { fasciitis }\end{array}$ & & $\begin{array}{l}4 \text { domains; } \\
\text { clinician }\end{array}$ & & \\
\hline & & & Location: Australia & & & & \\
\hline \multirow[t]{4}{*}{$\begin{array}{l}\text { Foot Function Index/ } \\
\text { Foot Impact Scale } \\
\text { (FIS), } 2005\end{array}$} & \multirow[t]{4}{*}{$\begin{array}{l}\text { Helliwell, P } \\
\text { [29] }\end{array}$} & \multirow[t]{4}{*}{$\begin{array}{l}\text { Validation with } \\
\text { Health } \\
\text { Assessment } \\
\text { Questionnaire } \\
\text { (HAQ), FFI, and } \\
\text { Manchester } \\
\text { Foot Disability } \\
\text { Questionnaires } \\
\text { (MFDQ) }\end{array}$} & N: 148 (34 male) & $\begin{array}{l}\text { Item Response } \\
\text { Theory }\end{array}$ & FIS & $\begin{array}{l}\text { Visual } \\
\text { Analog } \\
\text { Scale }\end{array}$ & $\begin{array}{l}\text { FIS items were } \\
\text { derived from RA } \\
\text { patients (consisted of } \\
\text { impairment/shoes } \\
\text { and activities/ } \\
\text { participation } \\
\text { subscales), with good } \\
\text { clinimetric properties. }\end{array}$ \\
\hline & & & $\begin{array}{l}\text { Mean age: } 61.7 \\
\text { (Range 28-89) }\end{array}$ & & 51 items & & Conclusion: Positive. \\
\hline & & & Dx: RA Foot Pain & & 2 domains & & \\
\hline & & & Location: UK & & Patient & & \\
\hline \multirow[t]{4}{*}{$\begin{array}{l}\text { Foot Function Index, } \\
2005\end{array}$} & \multirow[t]{4}{*}{ Agel, J [25] } & \multirow[t]{4}{*}{$\begin{array}{l}\text { Reliability and } \\
\text { validity tests in } \\
\text { specific } \\
\text { population } \\
\text { with moderate } \\
\text { to high } \\
\text { physical } \\
\text { function }\end{array}$} & $\begin{array}{l}\text { N: } 54 \text { ( } 22 \text { male, } 6 \\
\text { unknown) }\end{array}$ & $\begin{array}{l}\text { Correlation } \\
\text { statistics }\end{array}$ & $\begin{array}{l}\text { Foot Function } \\
\text { Index }\end{array}$ & $\begin{array}{l}\text { Likert } \\
\text { Scale }\end{array}$ & $\begin{array}{l}\text { Use of Foot Function } \\
\text { Index in non-systemic } \\
\text { foot and ankle } \\
\text { problems requires } \\
\text { removal of } 2 \text { items } \\
\text { each from pain and } \\
\text { disability, judged not } \\
\text { applicable for this } \\
\text { condition. }\end{array}$ \\
\hline & & & $\begin{array}{l}\text { Mean age: } 52.8 \\
(\mathrm{SD}=12.3) \text { (Range } \\
19-74)\end{array}$ & & 23 items & & Conclusion: Positive. \\
\hline & & & $\begin{array}{l}\text { Dx: Non-traumatic } \\
\text { foot/ankle } \\
\text { complaints }\end{array}$ & & 3 domains & & \\
\hline & & & Location: USA & & & & \\
\hline \multirow[t]{4}{*}{$\begin{array}{l}\text { Foot Function Index, } \\
2005\end{array}$} & \multirow[t]{4}{*}{$\begin{array}{l}\text { Shrader, JA } \\
\text { [28] }\end{array}$} & \multirow[t]{4}{*}{$\begin{array}{l}\text { Reliability and } \\
\text { validity } \\
\text { measures of } \\
\text { navicular joint } \\
\text { deformity vs. } \\
\text { clinical findings }\end{array}$} & $\mathrm{N}: 20$ (0 male) & & $\begin{array}{l}\text { Foot Function } \\
\text { Index }\end{array}$ & $\begin{array}{l}\text { Visual } \\
\text { Analog } \\
\text { Scale }\end{array}$ & $\begin{array}{l}\text { Foot Function Index } \\
\text { was used to measure } \\
\text { the foot health status } \\
\text { associated with joint } \\
\text { deformities. }\end{array}$ \\
\hline & & & $\begin{array}{l}\text { Mean age: } 55.4 \\
(\mathrm{SD}=11.4 \text { years); } \\
\mathrm{Dx}: \mathrm{RA} 12.7 \text { years } \\
(\mathrm{SD}=10.4)\end{array}$ & & Index 23 items & & Conclusion: Positive. \\
\hline & & & $\begin{array}{l}\text { Dx: Navicular joint } \\
\text { dropped and foot } \\
\text { pain }\end{array}$ & & 3 domains & & \\
\hline & & & Location: USA & & & & \\
\hline \multirow[t]{4}{*}{$\begin{array}{l}\text { Foot Function Index-R } \\
\text { with Foot Function } \\
\text { Index, } 2006\end{array}$} & \multirow[t]{4}{*}{$\begin{array}{l}\text { Budiman-Mak, } \\
\text { E [11] }\end{array}$} & \multirow[t]{4}{*}{$\begin{array}{l}\text { Instrument } \\
\text { Development }\end{array}$} & N: 97 (90 male) & $\begin{array}{l}\text { Item Response } \\
\text { Theory }\end{array}$ & $\begin{array}{l}\text { Foot Function } \\
\text { Index }\end{array}$ & $\begin{array}{l}\text { Likert scale } \\
\text { (replaced } \\
\text { Visual } \\
\text { Analog } \\
\text { Scale) }\end{array}$ & $\begin{array}{l}\text { Foot Function Index-R } \\
\text { had } 3 \text { domains, plus } \\
4^{\text {th }} \text { psychosocial } \\
\text { domain added to } \\
\text { assess quality of life. }\end{array}$ \\
\hline & & & $\begin{array}{l}\text { Mean age: } 69 \\
\text { (range: } 38-88 \text { ) }\end{array}$ & & 68 items (long) & & Conclusion: Positive \\
\hline & & & $\begin{array}{l}\text { Dx: Chronic foot } \\
\text { and ankle } \\
\text { complain }\end{array}$ & & 34 items (short) & & \\
\hline & & & Location: USA & & & & \\
\hline
\end{tabular}


Table 5 Studies of foot function measures (Continued)

\begin{tabular}{|c|c|c|c|c|c|c|c|}
\hline & & & & & $\begin{array}{l}\text { Clinicians and } \\
\text { patients }\end{array}$ & & \\
\hline \multirow[t]{3}{*}{$\begin{array}{l}\text { Foot Function Index, } \\
2006\end{array}$} & \multirow[t]{3}{*}{ Bal, A [26] } & \multirow[t]{3}{*}{$\begin{array}{l}\text { Comparing } \\
\text { Foot Function } \\
\text { Index with } \\
\text { Health } \\
\text { Assessment } \\
\text { Questionnaires } \\
\text { (HAQ) \& SFC }\end{array}$} & N: 78 (11 male) & \multirow[t]{3}{*}{$\begin{array}{l}\text { Correlation } \\
\text { statistics }\end{array}$} & $\begin{array}{l}\text { Foot Function } \\
\text { Index }\end{array}$ & \multirow[t]{3}{*}{$\begin{array}{l}\text { Visual } \\
\text { Analog } \\
\text { Scale }\end{array}$} & $\begin{array}{l}\text { Strong correlations of } \\
\text { HAQ and Foot } \\
\text { Function Index scores, } \\
\text { HR and CV also } \\
\text { reflected in Foot } \\
\text { Function Index scores } \\
\text { and were highly } \\
\text { correlated with Rand } \\
36 \text { items Short Form } \\
\text { Health Survey (SF36). }\end{array}$ \\
\hline & & & $\begin{array}{l}\text { Mean age: } 50.65 \\
(\mathrm{SD}=10.7) ; \mathrm{RA} \\
\text { duration } 13.96 \\
(\mathrm{SD}=8.09)\end{array}$ & & 23 items & & Conclusion: Positive \\
\hline & & & Location: Turkey & & 3 Domains & & \\
\hline \multirow[t]{4}{*}{$\begin{array}{l}\text { Foot Function Index \& } \\
\text { SF36, } 2006\end{array}$} & \multirow[t]{4}{*}{$\begin{array}{l}\text { SooHoo, N } \\
{[27]}\end{array}$} & \multirow[t]{4}{*}{$\begin{array}{l}\text { Validity test in } \\
\text { foot health } \\
\text { and general } \\
\text { physical health }\end{array}$} & $\mathrm{N}: 69$ (25 male) & \multirow[t]{4}{*}{$\begin{array}{l}\text { Correlation } \\
\text { statistics }\end{array}$} & $\begin{array}{l}\text { Foot Function } \\
\text { Index }\end{array}$ & \multirow[t]{4}{*}{$\begin{array}{l}\text { Visual } \\
\text { Analog } \\
\text { Scale }\end{array}$} & $\begin{array}{l}\text { The } 3 \text { domains of } \\
\text { Foot Function Index } \\
\text { demonstrated } \\
\text { moderate-high } \\
\text { correlation with SF36, } \\
\text { thus it was reasonable } \\
\text { to use Foot Function } \\
\text { Index to monitor } \\
\text { outcomes. }\end{array}$ \\
\hline & & & $\begin{array}{l}\text { Mean Age: } 46 \\
\text { (Range 16-82) }\end{array}$ & & 23 items & & Conclusion: Positive. \\
\hline & & & $\begin{array}{l}\text { Dx: Foot \& Ankle } \\
\text { disorder }\end{array}$ & & 3 domains & & \\
\hline & & & Location: USA & & & & \\
\hline \multirow[t]{4}{*}{$\begin{array}{l}\text { Foot Function Index \& } \\
\text { American Orthopedic } \\
\text { Foot and Ankle } \\
\text { Society (AOFAS) } \\
\text { hallux module, } 2006\end{array}$} & \multirow[t]{4}{*}{$\begin{array}{l}\text { Baumhauer, JF } \\
{[32]}\end{array}$} & \multirow[t]{4}{*}{$\begin{array}{l}\text { Reliability and } \\
\text { validity of test, } \\
\text { compared with } \\
\text { Foot Function } \\
\text { Index }\end{array}$} & N:11 (1 male) & \multirow[t]{4}{*}{$\begin{array}{l}\text { Correlation } \\
\text { statistics }\end{array}$} & \multirow[t]{4}{*}{$\begin{array}{l}\text { AOFAS hallux \& } \\
\text { lesser toes } \\
\text { module }\end{array}$} & \multirow[t]{4}{*}{$\begin{array}{l}\text { Numeric } \\
\text { rating } \\
\text { scale }\end{array}$} & $\begin{array}{l}\text { Only AOFAS hallux for } \\
\text { pain correlated with } \\
\text { Foot Function Index } \\
\text { pain scale. }\end{array}$ \\
\hline & & & $\begin{array}{l}\text { Mean age: } 54 \\
\text { (Range: } 40-72 \text { ) }\end{array}$ & & & & Conclusion: Positive. \\
\hline & & & $\begin{array}{l}\text { Dx: RA without } \\
\text { foot complaints }\end{array}$ & & & & \\
\hline & & & Location: USA & & & & \\
\hline \multirow[t]{3}{*}{$\begin{array}{l}\text { Foot Function Index, } \\
2006\end{array}$} & \multirow[t]{3}{*}{$\begin{array}{l}\text { Van der } \\
\text { Leeden, M } \\
\text { [30] }\end{array}$} & \multirow[t]{3}{*}{$\begin{array}{l}\text { Measure } \\
\text { forefoot } \\
\text { damage }\end{array}$} & $\mathrm{N}: 62$ (15 male) & \multirow[t]{3}{*}{$\begin{array}{l}\text { Correlation } \\
\text { Statistics }\end{array}$} & \multirow[t]{3}{*}{$\begin{array}{l}\text { Validation with } \\
\text { Western Ontario } \\
\text { Mac Masters } \\
\text { Universities } \\
\text { Osteoarthritis } \\
\text { Index (WOMAC) } \\
\text { and Disease } \\
\text { Activity in 44 RA } \\
\text { joints (DAS-44) }\end{array}$} & \multirow[t]{3}{*}{$\begin{array}{l}\text { Numeric } \\
\text { rating } \\
\text { scale }\end{array}$} & $\begin{array}{l}\text { Foot Function Index } \\
\text { function subscale } \\
\text { correlated with } \\
\text { WOMAC and DAS- } 44 \text {. } \\
\text { Foot Function Index } \\
\text { pain score correlated } \\
\text { with forefoot pain. } \\
\text { Foot Function Index } \\
\text { function score } \\
\text { correlated with hind } \\
\text { foot problem. }\end{array}$ \\
\hline & & & $\begin{array}{l}\text { Mean age: } 55.7 \\
(S D=13.11)\end{array}$ & & & & Conclusion: Positive. \\
\hline & & & $\begin{array}{l}\text { Dx: RA forefoot } \\
\text { complaints, } \\
\text { duration of } 96 \\
\text { months }\end{array}$ & & & & \\
\hline
\end{tabular}


Table $\mathbf{5}$ Studies of foot function measures (Continued)

\begin{tabular}{|c|c|c|c|c|c|c|c|}
\hline & & & $\begin{array}{l}\text { Location: } \\
\text { Netherlands }\end{array}$ & & & & \\
\hline \multirow[t]{4}{*}{$\begin{array}{l}\text { Foot Function Index, } \\
\text { American Orthopedic } \\
\text { Foot and Ankle } \\
\text { Society (AOFAS) } \\
\text { clinical rating } \\
\text { component, } 2007\end{array}$} & \multirow[t]{4}{*}{ Ibrahim, T [33] } & \multirow{4}{*}{$\begin{array}{l}\text { Testing the } \\
\text { criterion } \\
\text { validity of } \\
\text { clinical rating } \\
\text { components of } \\
\text { AOFAS with } \\
\text { Foot Function } \\
\text { Index }\end{array}$} & N:45 (11 male) & \multirow[t]{4}{*}{$\begin{array}{l}\text { Correlation } \\
\text { Statistics }\end{array}$} & \multirow[t]{4}{*}{$\begin{array}{l}\text { Validity of } \\
\text { AOFAS scale }\end{array}$} & \multirow[t]{4}{*}{$\begin{array}{l}\text { Numeric } \\
\text { rating } \\
\text { scale }\end{array}$} & \multirow{2}{*}{$\begin{array}{l}\text { The scores of AOFAS } \\
\text { clinical ratings and } \\
\text { Foot Function Index } \\
\text { were moderately } \\
\text { correlated based on } \\
41 \% \text { response rate. } \\
\text { Conclusion: Positive. }\end{array}$} \\
\hline & & & $\begin{array}{l}\text { Mean age: } 55 \\
\text { years (range }=15 \text { - } \\
81 \text { ) }\end{array}$ & & & & \\
\hline & & & $\begin{array}{l}\text { Dx: Hallux } \\
\text { deformities }\end{array}$ & & & & \\
\hline & & & Location: UK & & & & \\
\hline \multirow[t]{5}{*}{$\begin{array}{l}\text { Foot Function Index,/ } \\
\text { Foot Function Index } \\
\text { Chinese (Taiwan), } \\
2008\end{array}$} & \multirow[t]{5}{*}{ WU, SH [36] } & \multirow[t]{5}{*}{$\begin{array}{l}\text { Reliability and } \\
\text { validity } \\
\text { measure of } \\
\text { PCS of SF-26, } \\
\text { Taiwan version; }\end{array}$} & $\begin{array}{l}\mathrm{N}: 50 \text { (planta } \\
\text { fasciitis); mean } \\
\text { age } 46.9(\mathrm{SD}=10.6)\end{array}$ & \multirow[t]{5}{*}{$\begin{array}{l}\text { Cross-cultural } \\
\text { adaptation }\end{array}$} & $\begin{array}{l}\text { Foot Function } \\
\text { Index }\end{array}$ & \multirow[t]{5}{*}{$\begin{array}{l}\text { Visual } \\
\text { Analog } \\
\text { Scale }\end{array}$} & $\begin{array}{l}\text { Foot Function Index } \\
\text { Taiwan Chinese } \\
\text { consisted of } 21 \text { items. } \\
\text { Could measure non- } \\
\text { traumatic and } \\
\text { traumatic foot and } \\
\text { ankle problems. The } \\
\text { floor score was 10\%, } \\
\text { in sample with } \\
\text { fractures. }\end{array}$ \\
\hline & & & $\begin{array}{l}\mathrm{N}: 29 \text { (ankle/foot } \\
\text { fracture); mean } \\
\text { age } 37.2(\mathrm{SD}=14.8) \\
25 \text { male }\end{array}$ & & 21 items & & Conclusion: Positive. \\
\hline & & & \multirow[t]{3}{*}{ Location: Taiwan } & & 3 domains & & \\
\hline & & & & & $\begin{array}{l}\text { The order of } \\
\text { items was } \\
\text { changed. }\end{array}$ & & \\
\hline & & & & & $\begin{array}{l}\text { Clinician and } \\
\text { patient }\end{array}$ & & \\
\hline \multirow[t]{4}{*}{$\begin{array}{l}\text { Foot Function Index, } \\
\text { Foot Function Index- } \\
\text { D, } 2008\end{array}$} & \multirow[t]{4}{*}{ Naal, FD [34] } & \multirow[t]{4}{*}{$\begin{array}{l}\text { Foot Function } \\
\text { Index-D, }\end{array}$} & N:53 (14 male) & \multirow[t]{4}{*}{$\begin{array}{l}\text { Cross-cultural } \\
\text { adaptation }\end{array}$} & $\begin{array}{l}\text { Foot Function } \\
\text { Index-D }\end{array}$ & \multirow[t]{4}{*}{$\begin{array}{l}\text { Numeric } \\
\text { rating } \\
\text { scale }\end{array}$} & $\begin{array}{l}\text { Foot Function Index } \\
\text { underwent German } \\
\text { translation. Foot } \\
\text { Function Index-D } \\
\text { added } 3 \text { new items } \\
\text { and revised } 8 \text { items of } \\
\text { the Foot Function } \\
\text { Index and had } \\
\text { demonstrated good } \\
\text { clinimetrics. }\end{array}$ \\
\hline & & & $\begin{array}{l}\text { Age: } 57.2 \\
(\mathrm{SD}=13.7) \text { Range } \\
(18=77)\end{array}$ & & $\begin{array}{l}\text { Index-D } 18 \text { items } \\
\text { (pain \& disability } \\
\text { subscales) }\end{array}$ & & Conclusion: Positive. \\
\hline & & & $\begin{array}{l}\text { Dx: Foot } \\
\text { complaints }\end{array}$ & & 2 domains & & \\
\hline & & & Location: Germany & & $\begin{array}{l}\text { Clinician and } \\
\text { patient }\end{array}$ & & \\
\hline
\end{tabular}


Table 6 Clinimetric properties of patient-reported foot function measures

\begin{tabular}{|c|c|c|c|c|c|c|c|}
\hline $\begin{array}{l}\text { Instrument; } \\
\text { author year }\end{array}$ & $\begin{array}{l}\text { Reliability e.g., IRT, CTT } \\
\text { ICC, kappa, } \\
\text { test-retest }\end{array}$ & $\begin{array}{l}\text { Cronbach's } \\
\text { alpha }\end{array}$ & $\begin{array}{l}\text { Instrument } \\
\text { /Domain N } \\
\text { items/ Item } \\
\text { generated } \\
\text { sources }\end{array}$ & $\begin{array}{l}\text { Validity (Face, content, } \\
\text { criterion or construct) } \\
\text { and other measures }\end{array}$ & $\begin{array}{l}\text { Response } \\
\text { to change }\end{array}$ & $\begin{array}{l}\text { Completion } \\
\text { time }\end{array}$ & $\begin{array}{l}\text { Sample N } \\
\text { diagnoses } \\
\text { conclusion }\end{array}$ \\
\hline \multirow{5}{*}{$\begin{array}{l}\text { FFl; Budiman } \\
\text { Mak, E [7] } 1991\end{array}$} & $\mathrm{CTT}$ & Total: 0.96 & $\mathrm{FFI}$ & Face: yes & Yes & 10 minutes & $N=87$ \\
\hline & ICC total: 0.87 & Pain: 0.70 & 23 items & $\begin{array}{l}\text { Criterion: } r=0.52 \mathrm{FFI} \text { total } \\
\text { scores vs } 50 \mathrm{ft} \text { walked }\end{array}$ & & & $\begin{array}{l}\text { Early } \\
\text { rheumatoid } \\
\text { arthritis }\end{array}$ \\
\hline & ICC (pain): 0.70 & $\begin{array}{l}\text { Disability: } 0.93 \\
\text { Activity }\end{array}$ & $\begin{array}{l}\text { Clinician and } \\
\text { patient }\end{array}$ & Construct: Yes & & & $\begin{array}{l}\text { Conclusion: } \\
\text { Positive }\end{array}$ \\
\hline & ICC (disability): 0.84 & Limitation 0.73 & & & & & \\
\hline & $\begin{array}{l}\text { ICC (activity limitation): } \\
0.81\end{array}$ & & & & & & \\
\hline \multirow{2}{*}{$\begin{array}{l}\text { FFI pain } \\
\text { subscale (R/L } \\
\text { foot); Saag, KG } \\
\text { [23] } 1996\end{array}$} & $\mathrm{CTT}$ & $0.94-0.96$ & $\begin{array}{l}\text { FFI side-to-side; } \\
\text { Clinician and } \\
\text { patient }\end{array}$ & Face: Yes & & & $\begin{array}{l}\mathrm{N}=63 \\
\text { Rheumatoid } \\
\text { foot pain }\end{array}$ \\
\hline & ICC: $0.79-0.89$ & & & Content: Yes & & & $\begin{array}{l}\text { Conclusion: } \\
\text { Positive }\end{array}$ \\
\hline \multirow{4}{*}{$\begin{array}{l}\mathrm{FFI} \text { and } \mathrm{AOS} ; \\
\text { Domsic, RT } \\
{[24] 1998}\end{array}$} & $\mathrm{CTT}$ & & AOS & $\begin{array}{l}\text { Criterion: AOS vs } \\
\text { WOMAC disability }\end{array}$ & & & $N=562$ \\
\hline & ICC: 0.97 & & $\begin{array}{l}18 \text { items; } \\
\text { Clinician }\end{array}$ & $r=0.65$ pain $r=0.79$ & & & $\begin{array}{l}\text { Dx: Ankle } \\
\text { Osteoarthritis }\end{array}$ \\
\hline & Pain: 0.95 & & & Construct: Yes & & & $\begin{array}{l}\text { Conclusion: } \\
\text { Positive }\end{array}$ \\
\hline & Disability: 0.94 & & & & & & \\
\hline \multirow[t]{7}{*}{$\begin{array}{l}\text { FFI \& FHSQ. } \\
\text { Bennet, PJ [9] } \\
1998\end{array}$} & $\mathrm{CTT}$ & $0.85-0.88$ & FHSQ & Criterion: Yes & & 3-5 minutes & $\begin{array}{l}\mathrm{N}=255 \mathrm{Dx}: \\
\text { Hallux valgus } \\
\text { osteoarthritis }\end{array}$ \\
\hline & ICC & Pain: 0.88 & 13 items & $\begin{array}{l}\text { Construct: Yes, } \\
\text { discriminant validity; } \\
\text { Goodness of Fit }\end{array}$ & & & $\begin{array}{l}\text { Conclusion: } \\
\text { Positive }\end{array}$ \\
\hline & $0.74-0.92$ & Function: 0.85 & $\begin{array}{l}\text { Clinician and } \\
\text { Patient }\end{array}$ & & & & \\
\hline & pain 0.86 & Footwear: 0.85 & & & & & \\
\hline & function 0.92 & Foothealth: 0.87 & & & & & \\
\hline & footwear 0.74 & & & & & & \\
\hline & foothealth 0.78 & & & & & & \\
\hline \multirow{5}{*}{$\begin{array}{l}\mathrm{FFI}(5 \mathrm{pt}) ; \\
\text { Kuyvenhoven, } \\
\text { MM [3] } 2002\end{array}$} & $\mathrm{CTT}$ & $0.88-0.94$ & $\mathrm{FFI}(5 \mathrm{pt})$ & Concurrent validity: Yes & Yes & & $N=206$ \\
\hline & ICC 0.64-0.79 & Total: 0.93 & 15 items & & & & $\begin{array}{l}\text { Dx: Non- } \\
\text { traumatic foot } \\
\text { complaint }\end{array}$ \\
\hline & Total: 0.76 & Pain: 0.88 & Clinician & & & & $\begin{array}{l}\text { Conclusion: } \\
\text { Positive }\end{array}$ \\
\hline & Pain: 0.64 & Disability: 0.92 & & & & & \\
\hline & Disability: 0.79 & & & & & & \\
\hline \multirow{4}{*}{$\begin{array}{l}\text { FFI \& FIS; } \\
\text { Helliwell,P [29] } \\
2005\end{array}$} & IRT & Not performed & FIS & Face: Yes & & & $N=192$ \\
\hline & ICC & & 51 items & Content: Yes & & & $\begin{array}{l}\text { Rheumatoid } \\
\text { arthritis }\end{array}$ \\
\hline & $\begin{array}{l}\text { Impairment/shoes: } 0.84 \\
\text { Activities/participation: } \\
\text { 0/96 }\end{array}$ & & 2 subscales & Construct: Yes & & & $\begin{array}{l}\text { Conclusion: } \\
\text { Positive }\end{array}$ \\
\hline & & & $\begin{array}{l}\text { clinician and } \\
\text { patient }\end{array}$ & Goodness of Fit & & & \\
\hline
\end{tabular}


Table 6 Clinimetric properties of patient-reported foot function measures (Continued)

FFl; Agel, J ICC

[25] 2005

\begin{tabular}{|c|c|}
\hline \multicolumn{2}{|c|}{ Total: 0.68} \\
\hline \multirow{4}{*}{$\begin{array}{l}\text { FFI-R; } \\
\text { Budiman-Mak, } \\
\text { E [11] } 2006\end{array}$} & $\begin{array}{l}\text { All subscale values were } \\
\text { significant at } .01 \text { level }\end{array}$ \\
\hline & IRT \\
\hline & Person reliability: 0.96 \\
\hline & Item reliability:0.93 \\
\hline \multirow[t]{3}{*}{$\begin{array}{l}\text { FFI \& SF 36: } \\
\text { SooHoo, NF } \\
\text { [27] } 2006\end{array}$} & $\begin{array}{l}\text { Pearson Correlation of } \\
\text { FFI to SF-36: Pain: }-0.10 \\
\text { to }-0.61 \text {; }\end{array}$ \\
\hline & Disability: -0.23 to -0.69 \\
\hline & $\begin{array}{l}\text { Activity limitation: }-0.23 \\
\text { to }-0.61\end{array}$ \\
\hline $\begin{array}{l}\text { FFI AOFAS; } \\
\text { Baumhaur, JF } \\
\text { [32] } 2006\end{array}$ & $\begin{array}{l}\text { ICC AOFAS Summary } \\
\text { Scores: Hallux } 0.95 \text { Lesser } \\
\text { toes: } 0.8 \text { Pearson's } \\
\text { correlations mean value } \\
\text { AOFAS Hallux vs. FFI: } \\
r=0.80, \text { AOFAS lesser toes } \\
\text { vs FFI: } r=0.69 ; \text { Pain } \\
\text { subscale AOFAS Hallux vs. } \\
\text { FFI summary score: } r=0.31\end{array}$ \\
\hline
\end{tabular}

Total: 0.95

Pain: 0.93

19 items items each from pain and difficulty subscales were deleted

Clinician

FFI-R

Long form (68 items); Short

form (34 items)

Clinician and

patient

Disability: 0.93

Activity

limitation: 0.88

Psychosocial:

0.86

$\mathrm{FFI}$

23 items

3 domains

FFI

23 items

3 domains

MID

ceasures were

reported; Minimal

important difference

(MID) was the focus of

this clinical measure
Landorf, KB

[101] 2007
$\mathrm{N}=54 \mathrm{FFI}$ was tested in nonsystemic or traumatic foot problems.

FFI was good for individuals with low level functioning.

Conclusion: Positive

Criterion: Yes

Construct: Yes

15 minutes

$\mathrm{N}=92$

Dx: Chronic foot and ankle problems

Minimal floor effect (4.5\%)

Conclusion: Positive

Goodness of Fit

Construct: Yes

$N=69$

Forefoot and hindfoot complaints

Moderate correlation between FFI and SF-36

Conclusion: Positive

Content: Yes $\mathrm{N}=11$
Criterion: Yes

Ceiling effect noted in lesser toe activity subscale
Rheumatoid Hallux and lesser toes

Conclusion: Positive

$N=175$

Plantar fasciitis

Function 7 , 
Table 6 Clinimetric properties of patient-reported foot function measures (Continued)

\begin{tabular}{|c|c|c|c|c|c|c|c|}
\hline & & & $\begin{array}{l}\text { FFl Pain 12, } \\
\text { Function 7, } \\
\text { Total } 7\end{array}$ & & & & $\begin{array}{l}\text { Conclusion: } \\
\text { Positive }\end{array}$ \\
\hline & & & VAS & & & & \\
\hline & & & Pain 9 & & & & \\
\hline \multirow[t]{3}{*}{$\begin{array}{l}\text { FFI, AOFAS; } \\
\text { Ibrahim, T [33] } \\
2007\end{array}$} & \multicolumn{2}{|l|}{$\begin{array}{l}\text { Test-retest AOFAS; pre } \\
\text { and post operation was } \\
\text { no different; } 41 \% \\
\text { response rate. Pearson } \\
\text { correlation with FFI was } \\
\text { - } 0.68 \text { for all the } \\
\text { subjective components } \\
\text { of AOFAS. Hallux module } \\
\text { subjective component } \\
\text { was }-0.46\end{array}$} & $\begin{array}{l}\text { AOFAS subjective } \\
\text { component; } \\
\text { Items dependent } \\
\text { on modules }\end{array}$ & Criterion: yes & Yes & & $\begin{array}{l}\mathrm{N}=45 \text { Foot } \\
\text { and ankle } \\
\text { problems }\end{array}$ \\
\hline & & & Clinician & Construct: Yes & & & $\begin{array}{l}\text { AOFAS } \\
\text { reliability and } \\
\text { validity was } \\
\text { tested. }\end{array}$ \\
\hline & & & & $\begin{array}{l}\text { Discriminant and } \\
\text { predictive validity }\end{array}$ & & & $\begin{array}{l}\text { Conclusion: } \\
\text { positive with } \\
\text { caution due to } \\
\text { several } \\
\text { limitations as } \\
\text { mentioned in } \\
\text { the paper. }\end{array}$ \\
\hline \multirow{7}{*}{$\begin{array}{l}\text { FFI, FFI Taiwan } \\
\text { Chinese; Wu, } \\
\text { SH [36] } 2008\end{array}$} & ICC & CA & & $\begin{array}{l}\text { Criterion: Yes Floor effect } \\
10 \%\end{array}$ & & & $N=79$ \\
\hline & Total 0.82 & Total 0.94 & & & & & $\begin{array}{l}\text { Traumatic } \\
\text { (fracture) non- } \\
\text { traumatic } \\
\text { plantar fasciitis } \\
\text { foot problems }\end{array}$ \\
\hline & Pain 0.74 & Pain 0.91 & & & & & $\begin{array}{l}\text { Conclusion: } \\
\text { positive with } \\
\text { caution, due } \\
\text { to limitations } \\
\text { (see article) }\end{array}$ \\
\hline & $\begin{array}{l}\text { Disability } 0.76 \text { activity } \\
\text { limitation } 0.88\end{array}$ & Disability 0.95 & & & & & \\
\hline & & $\begin{array}{l}\text { Activity } \\
\text { limitation } 0.75\end{array}$ & $\begin{array}{l}\text { Clinician and } \\
\text { patients }\end{array}$ & & & & \\
\hline & & $\begin{array}{l}\text { Pearson's } \\
\text { correlations }\end{array}$ & & & & & \\
\hline & & $\begin{array}{l}\text { FFl total with } \\
\text { SF } 36 r=-0.59 \\
\text { plan- tar } \\
\text { fasciitis } r= \\
-0.61 \text { ankle } \\
\text { fracture }\end{array}$ & & & & & \\
\hline \multirow[t]{2}{*}{$\begin{array}{l}\text { FFI, FFI- } \\
\text { German Naal } \\
\text { FD [34] } 2008\end{array}$} & ICC & CA total 0.97 & $\begin{array}{l}\text { FFI German } 18 \\
\text { items pain and } \\
\text { disability } \\
\text { subscales } 3 \text { items } \\
\text { were added to } \\
\text { the instrument } \\
\text { by patients }\end{array}$ & $\begin{array}{l}\text { Construct yes } \\
\text { Convergent validity FFI-G } \\
\text { vs PCS of SF-36, VAS } \\
\text { pain, disability UCLA } \\
\text { activity scale }\end{array}$ & Yes & $8.3 \mathrm{~min}$ & $N=53$ \\
\hline & Total 0.98 & pain 0.90 & $\begin{array}{l}\text { Clinician and } \\
\text { patients }\end{array}$ & & & & $\begin{array}{l}\text { Various foot } \\
\text { problems } \\
\text { required } \\
\text { surgery }\end{array}$ \\
\hline
\end{tabular}


Table 6 Clinimetric properties of patient-reported foot function measures (Continued)

\begin{tabular}{|c|c|c|c|c|c|}
\hline & Pain 0.97 & disability 0.95 & $\begin{array}{l}\text { Patient related } \\
\text { difficulty } 2.4 \text { of } \\
\text { rating scale } 1-10\end{array}$ & & \\
\hline & Disability 0.99 & $\begin{array}{l}\text { Cross cultural } \\
\text { adaptation } \\
\text { English to } \\
\text { German with } \\
\text { forward and } \\
\text { backward } \\
\text { protocol }\end{array}$ & & & $\begin{array}{l}\text { Conclusion: } \\
\text { positive }\end{array}$ \\
\hline \multirow[t]{3}{*}{$\begin{array}{l}\text { FFI-R; Rao S } \\
\text { [75] } 2009\end{array}$} & $\begin{array}{l}\text { This report is about } \\
\text { minimal detectible } \\
\text { change (MDC90) a } \\
\text { measure of clinical } \\
\text { importance. }\end{array}$ & & $\begin{array}{l}\text { FFI-R long } 68 \\
\text { items }\end{array}$ & MDC Total 5 Pain 5 & $\begin{array}{l}\mathrm{N}=22 \text { Orthoses } \\
\text { treatment in } \\
\text { mid foot pain }\end{array}$ \\
\hline & $\begin{array}{l}\text { A result of orthoses } \\
\text { intervention in midfoot } \\
\text { arthritis }\end{array}$ & & & Activity limitation 7 & $\begin{array}{l}\text { Conclusion } \\
\text { positive }\end{array}$ \\
\hline & & & & $\begin{array}{l}\text { Effect Size (ES) Total } 0.4 \\
\text { Pain } 0.6 \text { Activity } \\
\text { limitation } 0.4\end{array}$ & $\begin{array}{l}\text { MDC and ES } \\
\text { findings are } \\
\text { significant }\end{array}$ \\
\hline \multirow[t]{2}{*}{$\begin{array}{l}\text { FFI-R; Rao, S } \\
\text { [76] } 2010\end{array}$} & $\begin{array}{l}\text { A measure of clinical } \\
\text { importance of orthoses } \\
\text { intervention }\end{array}$ & & $\begin{array}{l}\mathrm{FFI-R} \mathrm{long} 68 \\
\text { items }\end{array}$ & $\begin{array}{l}\text { MDC Total 5, Pain } 5 \\
\text { Stiffness 6, Disanility 7, } \\
\text { Activity limitation } 7 \\
\text { Psychosocial } 7 \text { ES: Total } \\
\text { 0.7, Pain 0.84, Stiffness } \\
\text { 0.31, Disability 0.6, } \\
\text { Limitation 0.57, Psycho } \\
\text { social } 0.32\end{array}$ & $\begin{array}{l}\text { N } 30 \text { Mid foot } \\
\text { pain }\end{array}$ \\
\hline & & & & & $\begin{array}{l}\text { Conclusion } \\
\text { positive }\end{array}$ \\
\hline
\end{tabular}

\section{Response category analysis}

One requirement of the Rasch model is monotonicity: the requirement that, as person ability increases, the item step response function increases monotonically [20]. This means that choosing one categorical response over the prior-for example, moving from selecting " 2 = A little of the time," to selecting, "3 = Most of the time,"-increases with person ability. The proper functioning of the rating scale is examined using fit statistics, where: (i) outfit mean squares should be less than 2.0, (ii) average measures advance monotonically with each category, and (iii) step calibrations increase monotonically $[21,22]$.

\section{Results}

\section{Review of the literature}

Articles were obtained by using the search method defined in the Methods section; the search results included 752 articles from PubMed/MEDLINE and 640 articles from Embase. Further screening and selection procedures, as detailed in Figure 1, yielded 182 full-text articles. Of these, 53 articles were qualified for review. Twenty-five more articles were obtained from the search engine BioMedLib and from manual searches. A total of 78 articles qualified for this review, summarized and categorized into several tables,
Objective 1: Assessment of the prevalence of the FFI or FFI-R usage, population characteristics, and study locations

Among the 78 studies, we identified 4714 study participants for whom the FFI or FFI-R instrument had been used to measure foot health. This sample consisted of 1914 (41\%) male participants and 2688 (57\%) female participants, with a mean age of 48.58 years (SD, 4.9 years). There was a discrepancy of $2 \%$ between the sums of male and female participants, because gender was not reported in three studies (Table 1). Most of the participants were individuals and young adults, and a few studies involved juvenile participants. The types of studies included measurement practice studies $(n=17)$, surgery studies $(n=30)$, studies of orthotics $(n=19)$ or other clinical interventions $(n=4)$, and observational studies $(n=8)$. We identified 20 different diagnoses of foot and ankle pathology that were measured by FFI and FFI-R (Table 2). Among them, RA and plantar fasciitis were the two most common diagnoses and were also noted to be the most painful and disabling foot conditions. These studies were conducted by investigators in 17 countries; the United States, the Netherlands, and the United Kingdom were the three most frequent users of the FFI and FFI-R in studies involving foot and ankle problems (Table 3). 
Table 7 Studies using foot function measures in surgical interventions

\begin{tabular}{|c|c|c|c|c|c|c|c|}
\hline Instrument & $\begin{array}{l}1^{\text {st }} \\
\text { Author }\end{array}$ & Objective & $\begin{array}{l}\text { Population (N, Sex, Age, Dx, } \\
\text { location) }\end{array}$ & Analysis & $\begin{array}{l}\text { Items/ } \\
\text { Domains/ } \\
\text { Subscales }\end{array}$ & $\begin{array}{l}\text { Response } \\
\text { type }\end{array}$ & $\begin{array}{l}\text { Summary } \\
\text { evaluation }\end{array}$ \\
\hline \multirow[t]{3}{*}{$\begin{array}{l}\text { Foot } \\
\text { Function } \\
\text { Index (FFI), } \\
2000\end{array}$} & Lin, S [39] & $\begin{array}{l}\text { Validation of AOFAS } \\
\text { forefoot outcomes } \\
\text { of arthrodesis } \\
\text { surgery }\end{array}$ & $\begin{array}{l}\mathrm{N}: 16 \text { Mean age: } 44 \\
(\mathrm{SD}=13.96) 8 \text { male }\end{array}$ & Pre-post surgery & $\mathrm{FFI}$ & VAS & $\begin{array}{l}\text { Both FFI and AOFAS } \\
\text { scores were } \\
\text { improved at post } \\
\text { surgery. }\end{array}$ \\
\hline & & & $\begin{array}{l}\text { Dx: Tarsometatarso injury/ } \\
\text { degenerative arthritis }\end{array}$ & $\begin{array}{l}\text { Follow-up } 36 \\
\text { months (24-65 } \\
\text { months) }\end{array}$ & 23 items & & Conclusion: useful \\
\hline & & & Location: USA & $\begin{array}{l}\mathrm{FFI} \text { and } \mathrm{AOFAS} \\
\text { were applied at } \\
\text { pre-surgery and at } \\
\text { follow up }\end{array}$ & 3 domains & & \\
\hline \multirow[t]{4}{*}{ FFI, 2002} & $\begin{array}{l}\text { Watson, } \\
\text { TS [61] }\end{array}$ & $\begin{array}{l}\text { Validation with VAS } \\
\text { pain scale with SF- } \\
36 \text { short form in } \\
\text { plantar fasciotomy }\end{array}$ & $\begin{array}{l}\text { Group I N (control): } 75 \text { Mean } \\
\text { age: } 46 \text { (range: } 20-78) 14 \\
\text { male }\end{array}$ & $\begin{array}{l}\text { Retrospective } \\
\text { observational } \\
\text { Follow up duration } \\
26.4 \text { months }\end{array}$ & $\mathrm{FFI}$ & VAS & $\begin{array}{l}\text { FFI scores were } \\
\text { improved. }\end{array}$ \\
\hline & & & $\begin{array}{l}\text { Group II N (surgery): } 46 \text { Mean } \\
\text { age: } 46 \text { (Range: 25-78) } 9 \text { male }\end{array}$ & $\begin{array}{l}\text { Group II filled out } \\
\text { FFI and SF-26 at } \\
\text { post-surgery only }\end{array}$ & 23 items & & $\begin{array}{l}\text { FFI scores reflected } \\
\text { activities of daily } \\
\text { living. SF-36 s cores } \\
\text { reflection } \\
\text { satisfaction of } \\
\text { physical and role } \\
\text { model. }\end{array}$ \\
\hline & & & $\begin{array}{l}\text { Dx: Sub-Calcaneal pain } \\
\text { syndrome }\end{array}$ & $\begin{array}{l}\text { Validation with VAS } \\
\text { pain scale SF-36 } \\
\text { short form }\end{array}$ & 3 domains & & Conclusion: useful. \\
\hline & & & Location: USA & & & & \\
\hline \multirow[t]{6}{*}{ FFI, 2003} & $\begin{array}{l}\text { Mulcahy, } \\
\text { D [56] }\end{array}$ & $\begin{array}{l}\text { Surgery- } \\
\text { Reconstruction of } \\
\text { the forefoot; FFI } \\
\text { scores were used to } \\
\text { test if there was } \\
\text { correlation with } \\
\text { WOMAC, AOFAS } \\
\text { HMIP, and AOFAS } \\
\text { LMIP. }\end{array}$ & $\begin{array}{l}\text { N: } 7914 \text { male Mean age: } 59 \\
\text { (Range: 24-80) }\end{array}$ & $\begin{array}{l}\text { Retrospective } \\
\text { observational; } \\
\text { Follow up 6yrs. }+3 \\
\text { mo (6mo-19 years) }\end{array}$ & $\begin{array}{l}\text { FFI; } 23 \\
\text { items; } 3 \\
\text { domains }\end{array}$ & VAS & $\begin{array}{l}\text { FFI pain subscale } \\
\text { was used to } \\
\text { monitor pain in } \\
\text { both groups. }\end{array}$ \\
\hline & & & Dx: RA forefoot deformity & & & & Conclusion: useful \\
\hline & & & $\begin{array}{l}\text { Mean age of surgery: } 52 \text { years } \\
\text { (range: 23-79) }\end{array}$ & & & & \\
\hline & & & $\begin{array}{l}\text { Group } 1 \text { stable } 1^{\text {st }} \text { ray. (no } \\
\text { surgery) }\end{array}$ & & & & \\
\hline & & & Group 2: $1^{\text {st }}$ ray surgery & & & & \\
\hline & & & Location: Canada & & & & \\
\hline \multirow[t]{4}{*}{ FFI, 2004} & $\begin{array}{l}\text { Ibrahim T } \\
{[48]}\end{array}$ & $\begin{array}{l}\text { Surgery- MTP joint } \\
\text { replacement; } \\
\text { Validation of AOFAS } \\
\text { Hallux scale scores } \\
\text { with FFI scores from } \\
\text { those who did not } \\
\text { have surgery and } \\
\text { those who had } \\
\text { surgery }\end{array}$ & $\mathrm{N}: 8,1$ male & $\begin{array}{l}\text { Retrospective } \\
\text { observational; } \\
\text { Follow up for } 17 \\
\text { months }\end{array}$ & $\mathrm{FFI}$ & VAS & $\begin{array}{l}\text { Correlation } \\
\text { observed between } \\
\text { the scores of AOFAS } \\
\text { and FFI }\end{array}$ \\
\hline & & & Mean age: 58 (Range: 51-80) & & 23 items & & $\begin{array}{l}\text { Note: AOFAS Hallux } \\
\text { scale had not been } \\
\text { validated. }\end{array}$ \\
\hline & & & Dx. Hallux rigidus & & 3 domains & & Conclusion: useful \\
\hline & & & Location: UK & & & & \\
\hline
\end{tabular}


Table 7 Studies using foot function measures in surgical interventions (Continued)

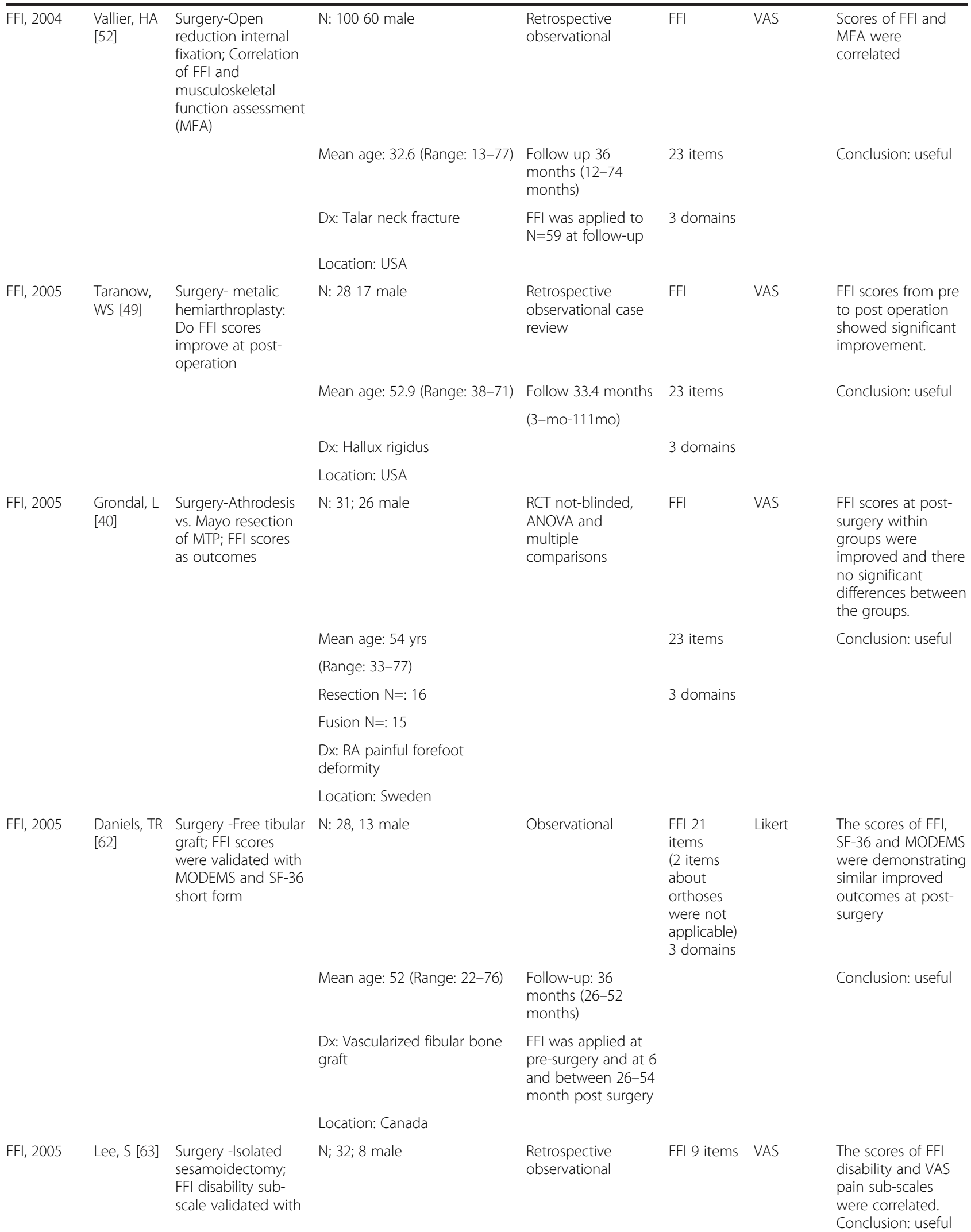


Table 7 Studies using foot function measures in surgical interventions (Continued)

\begin{tabular}{|c|c|c|c|c|c|c|c|}
\hline & & $\begin{array}{l}\text { VAS pain scale and } \\
\text { SF-36 short form }\end{array}$ & & & & & \\
\hline & & & Mean age: 37.2 (Range: 18-65) & 62 month & 1 domain: & & \\
\hline & & & & Post-op N=: 20 & $\begin{array}{l}\text { disability } \\
\text { scale }\end{array}$ & & \\
\hline & & & Dx: Hallux alignment & & & & \\
\hline & & & Location: USA & & & & \\
\hline \multirow[t]{4}{*}{$\mathrm{FFI}, 2006$} & $\begin{array}{l}\text { SooHoo, } \\
\text { NF [64] }\end{array}$ & $\begin{array}{l}\text { Surgery- Any type } \\
\text { of foot and ankle } \\
\text { surgery; Validating } \\
\text { AOFAS, SF-36 and } \\
\text { measuring Standard } \\
\text { Response Mean } \\
\text { (SRM) and effect } \\
\text { size (ES) }\end{array}$ & N: 25; 6 Male & $\begin{array}{l}\text { Pre-post surgery FFI } \\
\text { was applied at pre- } \\
\text { surgery and } 6 \\
\text { months post- } \\
\text { surgery }\end{array}$ & $\mathrm{FFI}$ & VAS & $\begin{array}{l}\text { Of the instruments } \\
\text { used, scores of the } \\
\text { pain subscale was } \\
\text { the only measure } \\
\text { reflecting high SRM } \\
(-0.83) \text { and ES } \\
(-0.86) \text {. Therefore, } \\
\text { pain is the most } \\
\text { important outcome } \\
\text { in studies regarding } \\
\text { chronic foot and } \\
\text { ankle pain. }\end{array}$ \\
\hline & & & Mean age: 40 (Range: 21-69) & & 23 items & & Conclusion: useful \\
\hline & & & $\begin{array}{l}\text { Dx: Chronic foot and ankle } \\
\text { conditions requiring surgery }\end{array}$ & & 3 domains & & \\
\hline & & & Location: USA & & & & \\
\hline \multirow[t]{4}{*}{ FFI, 2006} & $\begin{array}{l}\text { Van der } \\
\text { Krans, A } \\
{[41]}\end{array}$ & $\begin{array}{l}\text { Surgery- Calcaneal } \\
\text { Cuboid arthrodesis; } \\
\text { Correlation with } \\
\text { AOFAS clinical } \\
\text { rating index (CRI) of } \\
\text { the hind foot }\end{array}$ & N: 20; 4 Male & Pre-post surgery & $\begin{array}{l}\text { FFl-Dutch } \\
15 \text { items }\end{array}$ & $\begin{array}{l}5 \text {-point } \\
\text { verbal } \\
\text { scale }\end{array}$ & $\begin{array}{l}\text { FFI and CRI scores } \\
\text { showed significant } \\
\text { improvements }\end{array}$ \\
\hline & & & Mean age: 55 (Range: 30-66) & $\begin{array}{l}\text { Follow-up } 25 \\
\text { months (13-39 } \\
\text { months) }\end{array}$ & $\begin{array}{l}\text { Pain and } \\
\text { function } \\
\text { subscales }\end{array}$ & & Conclusion: useful \\
\hline & & & Dx: Flat foot & $\begin{array}{l}\text { FFI was applied at } \\
\text { pre-surgery and ad } \\
\text { follow-up }\end{array}$ & & & \\
\hline & & & Location: Netherlands & & & & \\
\hline \multirow[t]{4}{*}{ FFI, 2006} & $\begin{array}{l}\text { Harris, M } \\
{[53]}\end{array}$ & $\begin{array}{l}\text { Surgery- High } \\
\text { impact fracture } \\
\text { repair; Correlation } \\
\text { with } \\
\text { Musculoskeletal } \\
\text { function assessment } \\
\text { (MFA) }\end{array}$ & N: 76; 45 Male & $\begin{array}{l}\text { Pre-post surgery } \\
\text { follow up } 26 \\
\text { months ( } 24-38 \\
\text { months). FFI was } \\
\text { applied at pre- } \\
\text { surgery, } 6 \text { and } 12 \\
\text { weeks and at } 6 \\
\text { months by mail, } \\
\text { telephone, and was } \\
\text { self-administered. }\end{array}$ & $\mathrm{FFI}$ & VAS & $\begin{array}{l}\text { High FFI score } \\
\text { occurred in those } \\
\text { with the worse } \\
\text { fractures and } \\
\text { external fixation. } \\
\text { This is also reflected } \\
\text { in MFA scores. }\end{array}$ \\
\hline & & & Mean age: 45 (Range: 17-81) & & 23 items & & Conclusion: useful \\
\hline & & & $\begin{array}{l}\text { Dx: distal tibial plafond } \\
\text { fracture }\end{array}$ & & 3 domains & & \\
\hline & & & Location: USA & & & & \\
\hline \multirow[t]{2}{*}{ FFI, 2006} & $\begin{array}{l}\text { Stegman } \\
\text { M [42] }\end{array}$ & $\begin{array}{l}\text { Surgery-Triple } \\
\text { arthrodesis; } \\
\text { Correlation with } \\
\text { AOFAS hind foot } \\
\text { scores }\end{array}$ & N: $81 ; 38$ Male & Pre-post surgery & FFI Dutch & Likert & $\begin{array}{l}\text { FFI-5pt and AOFAS } \\
\text { hind foot scores } \\
\text { improved } 89 \% \text {. } \\
\text { However, patient } \\
\text { did not perceive the } \\
\text { benefit of the } \\
\text { procedure. }\end{array}$ \\
\hline & & & Mean age: 40.5 (Range: 14-79) & & 15 items & & Conclusion: useful \\
\hline
\end{tabular}


Table 7 Studies using foot function measures in surgical interventions (Continued)

\begin{tabular}{|c|c|c|c|c|c|c|c|}
\hline & & & & $\begin{array}{l}\mathrm{FFI} \text { applied at pre- } \\
\text { surgery and } 1 \mathrm{yr} \\
(1-4) \text { post surgery }\end{array}$ & & & \\
\hline & & & Dx: Hind foot disorders & & 2 domains & & \\
\hline & & & Location: Netherlands & & & & \\
\hline \multirow[t]{4}{*}{ FFI, 2007} & $\begin{array}{l}\text { Jung, HG } \\
{[45]}\end{array}$ & $\begin{array}{l}\text { Surgery-Fusion of } \\
\text { tarso metatarso- } \\
\text { joint; Correlation } \\
\text { with SF-36, AOFAS }\end{array}$ & N: 67; 12 Male & $\begin{array}{l}\text { Retrospective } \\
\text { observational }\end{array}$ & $\mathrm{FFI}$ & VAS & $\begin{array}{l}\text { Scores of the FFI, } \\
\text { SF-36 AOFAS and } \\
\text { VAS pain scale were } \\
\text { markedly improved } \\
\text { at post-surgery }\end{array}$ \\
\hline & & & Mean age: 60.2 (Range: $35-84$ ) & $\begin{array}{l}\text { Follow for } 40.6 \\
\text { months }\end{array}$ & 23 items & & Conclusion: useful \\
\hline & & & $\begin{array}{l}\text { Dx: Non-traumatic } \\
\text { osteoarthritis of the tarso- } \\
\text { meta-tarso joints }\end{array}$ & $\begin{array}{l}\text { FFI applied at post- } \\
\text { surgery }\end{array}$ & 3 domains & & \\
\hline & & & Location: USA & & & & \\
\hline \multirow[t]{4}{*}{ FFI, 2008} & $\begin{array}{l}\text { Vesely, R } \\
\text { [43] }\end{array}$ & $\begin{array}{l}\text { Surgery - Tibio } \\
\text { Calcaneal } \\
\text { arthrodesis; } \\
\text { Correlation with } \\
\text { ankle-hind foot } \\
\text { score }\end{array}$ & N: 20; 16 Male & $\begin{array}{l}\text { Retrospective } \\
\text { observational }\end{array}$ & $\mathrm{FFI}$ & VAS & $\begin{array}{l}\text { The scores of FFI } \\
\text { and ankle hind foot } \\
\text { were improved. }\end{array}$ \\
\hline & & & Mean age: 58.7 (Range: 23-72) & $\begin{array}{l}\mathrm{FFI} \text { applied at post- } \\
\text { surgery, time } \\
\text { unknown }\end{array}$ & 23 items & & Conclusion: useful \\
\hline & & & $\begin{array}{l}\text { Dx: Traumatic arthritis of the } \\
\text { ankles }\end{array}$ & $\begin{array}{l}\text { Article in Czech } \\
\text { with English } \\
\text { abstract. }\end{array}$ & 3 domains & & \\
\hline & & & Location: Czech Republic & & & & \\
\hline \multirow[t]{4}{*}{ FFI, 2008} & $\begin{array}{l}\text { Stropek, S } \\
\text { [37] }\end{array}$ & $\begin{array}{l}\text { Surgery- } \\
\text { arthroscopy }\end{array}$ & N: 26; 6 Male & $\begin{array}{l}\text { Pre-post surgery } \\
\text { observational }\end{array}$ & $\mathrm{FFI}$ & VAS & $\begin{array}{l}\text { FFI pain scale scores } \\
\text { were markedly } \\
\text { improved at post } \\
\text { surgery in } 79 \% \text { of } \\
\text { the patients }\end{array}$ \\
\hline & & & Age: male: 45; female: 49 & $\begin{array}{l}\text { FFI applied at pre- } \\
\text { surgery and at } 3 \\
\text { month follow-up }\end{array}$ & Pain scale & & Conclusion: useful \\
\hline & & & Dx: Calcaneal spur & & 9 items & & \\
\hline & & & Location: Czech Republic & & & & \\
\hline \multirow[t]{4}{*}{$\mathrm{FFI}, 2008$} & $\begin{array}{l}\text { Schutte, } \\
\text { BG [50] }\end{array}$ & $\begin{array}{l}\text { Surgery-Total ankle } \\
\text { replacement; pain } \\
\text { and function } \\
\text { outcome measure }\end{array}$ & N: 47; 16 Males & Pre-post surgery & FFI-Dutch & Likert & $\begin{array}{l}\text { Total scores } \\
\text { improved at post- } \\
\text { surgery }\end{array}$ \\
\hline & & & Mean age: 57.1 (range 37-81) & $\begin{array}{l}\text { FFI applied at pre- } \\
\text { surgery and at } \\
\text { follow up }\end{array}$ & 18 items & & Conclusion: useful \\
\hline & & & Dx: Ankle joint deformity & $\begin{array}{l}\text { Duration of follow } \\
\text { up } 28 \text { months } \\
\text { (range 12-67) }\end{array}$ & $\begin{array}{l}\text { Pain and } \\
\text { difficulty } \\
\text { subscales }\end{array}$ & & \\
\hline & & & Location: Netherlands & & & & \\
\hline FFI, 2008 & $\begin{array}{l}\text { Ward, CM } \\
\text { [57] }\end{array}$ & $\begin{array}{l}\text { Surgery- } \\
\text { Reconstruction; } \\
\text { Validation of SF } 26 \\
\text { with FFI }\end{array}$ & N: 25; 14 Male & Pre-post surgery & $\mathrm{FFI}$ & VAS & $\begin{array}{l}\text { At follow up the FFI } \\
\text { scores were in the } \\
\text { mid-range. The } \\
\text { scores for smokers } \\
\text { were worse than } \\
\text { non-smokers, } \\
\text { females were worse }\end{array}$ \\
\hline
\end{tabular}


than males. FFI activity limitation and disability scores were correlated with SF-36 physical component scores.
Mean age: 15 (Range: 8.7-25)

applied at mean age of 41.5 years after 26.1 yrs follow-

up

Dx: Flexible Cavovarus Charcot Marie-Tooth

Location: USA

Castellani, Surgery-Fixation C [65] with cannulation osteosynthesis; Outcomes of an intervention

FFI, $2009 \quad$ Bonnin,
MP [51]

Surgery - Total ankle arthoplasty; Correlations of FAAM (foot and ankle ability measure)

$\begin{array}{lll}\text { FFI, } 2009 \quad \text { Potter, MQ } & \text { Surgery- } \\ & \text { Intraarticular } \\ & \text { fracture of the } \\ & \text { Calcaneus; } \\ & \text { Correlation with } \\ & \text { AOFAS hind foot } \\ & \text { scores }\end{array}$

FFI, 2010 Aurich, M Surgery[66] Arthroscopic chondrocyte implant; Correlation with AOFAS hind foot scores and foot and ankle module of the Academy of Orthopedic Surgeon (AAOS)

Location: France Core Scale of the

\author{
N: 21; 11 Male
}

Dx: Transitional fracture of distal tibia

Age 13.7 (1.4)

Location: Austria

N: 140; 50 Male

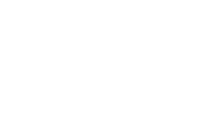

Mean age: 60.9 (Range: 26-90)

Dx: OA: 100 RA: 40
N: 73; 52 Male
Retrospective
observational FFI
applied at follow up
of 12.8 years
(5-18.5)

23 items
3 domains

$\mathrm{FFI}$

VAS

VAS

at pre-surgery

applied, and also at

$53.8 \pm 29$ months

(12-125) post-

surgery

\section{Dx: Calcaneal fracture}

Location: USA

N: 18; 13 Male

Retrospective observational FFI was applied at prearthroscopy and at follow-up, with mean duration of 19 months
23 items

3 domains

FFI 18 Likert items; Pain and

function subscales
Conclusion: useful

At follow-up 3 of the 21 (14\%) had poor FFI disability scores

Conclusion: useful

FFI pain scores were no different between $\mathrm{OA}$ and RA groups. The FFI scores were improved and were similar to that of FAAM.

Conclusion: useful

Scored of FFI, AOFAS hind foot and Calcaneal scores were correlated.

Conclusion: useful

FFI scores improved comparable with those of AOFAS results and Core Scale scores 


\begin{tabular}{|c|c|c|c|c|c|c|c|}
\hline & & & & & & & $\begin{array}{l}\text { caution in individual } \\
\text { whose. functional } \\
\text { level is better than } \\
\text { the level of activities } \\
\text { of daily living. }\end{array}$ \\
\hline & & & $\begin{array}{l}\text { Dx: Osteochondral lesion of } \\
\text { talus/tibia }\end{array}$ & & & & Conclusion: useful \\
\hline & & & Location:Australia & & & & \\
\hline \multirow[t]{4}{*}{ FFI, 2010} & \multirow[t]{4}{*}{$\begin{array}{l}\text { Van der } \\
\text { Heide, HJL } \\
{[59]}\end{array}$} & \multirow[t]{4}{*}{$\begin{array}{l}\text { Surgery-Correction } \\
\text { pes cavo varus; } \\
\text { Validation AOFAS } \\
\text { lesser toe module }\end{array}$} & N: 39; 6 Male & $\begin{array}{l}\text { Pre-post surgery; FFI } \\
\text { applied at pre- } \\
\text { surgery and } 40 \\
\text { month post-surgery }\end{array}$ & FFI-Dutch & VAS & $\begin{array}{l}\text { FFI pain and } \\
\text { function scores } \\
\text { improved post- } \\
\text { surgery }\end{array}$ \\
\hline & & & Mean age: 59 (Range: 29-81) & & & & Conclusion: useful \\
\hline & & & Dx: RA lesser MTP & & 23 items & & \\
\hline & & & Location: Netherlands & & 3domains & & \\
\hline \multirow[t]{4}{*}{$\begin{array}{l}\text { FFI- Dutch, } \\
2010\end{array}$} & \multirow[t]{4}{*}{$\begin{array}{l}\text { Kroon, M } \\
{[60]}\end{array}$} & \multirow[t]{4}{*}{$\begin{array}{l}\text { Surgery-Correction } \\
\text { pes cavo varus; } \\
\text { Validation AOFAS } \\
\text { hind foot scale }\end{array}$} & N: 15; 8 Male & \multirow[t]{3}{*}{$\begin{array}{l}\text { Pre-post surgery FFI } \\
\text { applied at pre and } \\
50 \text { month post } \\
\text { surgery }\end{array}$} & FFI-Dutch & \multirow[t]{3}{*}{ Likert } & $\begin{array}{l}\text { Pain and function } \\
\text { scores improved } \\
\text { post surgery }\end{array}$ \\
\hline & & & Mean age:40 (SD 14) & & 18 items & & Conclusion: useful \\
\hline & & & Dx: Cavo varus foot deformity & & $\begin{array}{l}\text { Pain and } \\
\text { function } \\
\text { subscales }\end{array}$ & & \\
\hline & & & Location: The Netherlands & & & & \\
\hline \multirow[t]{7}{*}{ FFI, 2010} & \multirow{7}{*}{$\begin{array}{l}\text { Van } \\
\text { Doeselaar, } \\
\text { DJ [46] }\end{array}$} & \multirow{7}{*}{$\begin{array}{l}\text { Surgery-Fusion of } \\
\text { MTP; Correlation } \\
\text { with VAS pain and } \\
\text { satisfaction }\end{array}$} & $\mathrm{N}: 62$ & \multirow{7}{*}{$\begin{array}{l}\text { Pre-post surgery; FFI } \\
\text { applied at pre- } \\
\text { surgery and } 12 \\
\text { month post-surgery }\end{array}$} & \multirow{7}{*}{$\begin{array}{l}\text { FFI Dutch; } \\
18 \text { items }\end{array}$} & \multirow{7}{*}{ Likert } & \multirow{2}{*}{$\begin{array}{l}\text { FFI-5 pts scores } \\
\text { were improved. }\end{array}$} \\
\hline & & & 2 groups & & & & \\
\hline & & & Dx: $\mathrm{H}$ rigidus; $\mathrm{N}: 27 ; 9$ Male & & & & Conclusion: useful \\
\hline & & & Mean age: 58 (Range: 42-72) & & & & \\
\hline & & & Dx: H valgus; N: 35; 6 Male & & & & \\
\hline & & & Mean age: 61 (Range: 37-76) & & & & \\
\hline & & & Location: Netherlands & & & & \\
\hline \multirow[t]{4}{*}{ FFI, 2010} & \multirow[t]{4}{*}{$\begin{array}{l}\text { Doets, HC } \\
{[44]}\end{array}$} & \multirow[t]{4}{*}{$\begin{array}{l}\text { Surgery-Salvage } \\
\text { arthrodesis for failed } \\
\text { TAA; Correlating } \\
\text { with AOFAS and } \\
\text { VAS pain scale }\end{array}$} & N: 18; 4 Male & \multirow[t]{3}{*}{$\begin{array}{l}\text { Retrospective } \\
\text { observational FFI } \\
\text { applied at follow } \\
\text { up, 3-12 years }\end{array}$} & FFI-Dutch & \multirow[t]{3}{*}{$\begin{array}{l}5 \text { point } \\
\text { rating } \\
\text { scale }\end{array}$} & $\begin{array}{l}\text { FFI scores improved } \\
\text { similar to that of } \\
\text { AOFAS, VAS pain, } \\
\text { disability and } \\
\text { satisfaction measure }\end{array}$ \\
\hline & & & Mean age: 55 (Range: 27-76) & & 15 items & & Conclusion: useful \\
\hline & & & Dx: Failed TAA & & $\begin{array}{l}\text { Pain and } \\
\text { function } \\
\text { subscales }\end{array}$ & & \\
\hline & & & Location: Netherlands & & & & \\
\hline \multirow[t]{4}{*}{$\mathrm{FFI}, 2010$} & \multirow[t]{4}{*}{ Niki, H [47] } & \multirow[t]{4}{*}{$\begin{array}{l}\text { Surgery-TMT fusion } \\
\text { and osteotomy; } \\
\text { Concurrent } \\
\text { assessment of FFI } \\
\text { and SF-36 and } \\
\text { Japanese Society for } \\
\text { Surgery of the Foot } \\
\text { and Ankle Score }\end{array}$} & N: 30; 1 Male & $\begin{array}{l}\text { Pre-post surgery FFI } \\
\text { was applied at pre- } \\
\text { surgery and at } 36 \\
\text { mos follow-up }\end{array}$ & $\mathrm{FFI}$ & \multirow[t]{4}{*}{ VAS } & $\begin{array}{l}\text { The scores of all } \\
\text { instruments were } \\
\text { improved at post- } \\
\text { surgery. }\end{array}$ \\
\hline & & & Mean age: 53.6 (Range: 45-67) & & 23 items & & Conclusion: useful \\
\hline & & & Dx: RA fore-foot deformity & & 3 domains & & \\
\hline & & & Location: Japan & & & & \\
\hline
\end{tabular}


Table 7 Studies using foot function measures in surgical interventions (Continued)

\begin{tabular}{|c|c|c|c|c|c|c|c|}
\hline \multirow[t]{4}{*}{$\mathrm{FFI}, 2010$} & $\begin{array}{l}\text { Schlegel, } \\
\text { UJ [58] }\end{array}$ & $\begin{array}{l}\text { Surgery-Club foot } \\
\text { correctional; Post- } \\
\text { surgery foot health } \\
\text { assessment }\end{array}$ & N: 98; 72 Male & $\begin{array}{l}\text { Retrospective } \\
\text { observational FFI } \\
\text { was applied at } 8.2 \\
\text { years (0-11.2); Post } \\
\text { surgery N: } 46 \text { (50\%) }\end{array}$ & $\mathrm{FFI}$ & VAS & $\begin{array}{l}\text { FFI scores indicated } \\
\text { good foot health. }\end{array}$ \\
\hline & & & $\begin{array}{l}\text { Mean follow-up: 4.5M (Range: } \\
\text { 1-68) }\end{array}$ & & 23 items & & Conclusion: useful \\
\hline & & & Dx: Club foot & & 3 domains & & \\
\hline & & & Location: Germany & & & & \\
\hline \multirow[t]{7}{*}{ FFI, 2010} & $\begin{array}{l}\text { Gaskill, T } \\
{[55]}\end{array}$ & $\begin{array}{l}\text { Surgery- Internal } \\
\text { fixation of the } \\
\text { instraarticular } \\
\text { Calcaneal fracture; } \\
\text { Concurrent } \\
\text { evaluation with } \\
\text { OAFAS hind foot }\end{array}$ & N: 146; 99 Male & $\begin{array}{l}\text { Retrospective } \\
\text { observational FFI } \\
\text { was applied at post- } \\
\text { surgery } 8.98 \text { years }\end{array}$ & $\mathrm{FFI}$ & VAS & $\begin{array}{l}\text { FFI scores of Group } \\
1 \text { were better than } \\
\text { Group } 2 \text { at post } \\
\text { surgery. }\end{array}$ \\
\hline & & & $\begin{array}{l}\text { Group } 1<50 \text { yrs; N: 99; } 65 \\
\text { male }\end{array}$ & & 23 items & & Conclusion: useful \\
\hline & & & Mean age: 36 (Age range) & & 3 domains & & \\
\hline & & & $\begin{array}{l}\text { Group } 2>50 \text { years; } N \text { : } 47 ; 33 \\
\text { male }\end{array}$ & & & & \\
\hline & & & Mean age: 58 (Range: 50-84) & & & & \\
\hline & & & Dx: Calcaneal fracture & & & & \\
\hline & & & Location: USA & & & & \\
\hline \multirow[t]{6}{*}{ FFI, 2010} & $\begin{array}{l}\text { Eberl, R } \\
{[67]}\end{array}$ & $\begin{array}{l}\text { Surgery- Various } \\
\text { surgical techniques } \\
\text { were applied; Post } \\
\text { surgery outcomes }\end{array}$ & N: 24; 18 Male & $\begin{array}{l}\text { Retrospective } \\
\text { observational }\end{array}$ & $\mathrm{FFI}$ & VAS & $\begin{array}{l}\text { FFI scores improved } \\
\text { in both groups. } \\
\text { Group } 1 \text { scored } \\
\text { better than Group } 2 .\end{array}$ \\
\hline & & & $\begin{array}{l}\text { Mean age: } 13.2 \text { (Range: 5-17 } \\
\text { yrs) }\end{array}$ & $\begin{array}{l}\text { Follow-up } 3.2 \text { years } \\
\text { ( } 7 \text { months-8.2 years) }\end{array}$ & 23 items & & $\begin{array}{l}\text { Limitation: The } \\
\text { author stated that } \\
\text { use of self-report in } \\
\text { instrument in } \\
\text { children may result } \\
\text { in spurious } \\
\text { outcomes, due to } \\
\text { their pronounced } \\
\text { potential for } \\
\text { compensation. }\end{array}$ \\
\hline & & & $\begin{array}{l}\text { Group } 1<12 \text { years; N: 9; Age : } \\
9.2\end{array}$ & $\begin{array}{l}\text { FFI applied at } \\
\text { follow up }\end{array}$ & 3 domains & & Conclusion: useful \\
\hline & & & $\begin{array}{l}\text { Group } 2>12 \text { years; N: 15; Age: } \\
14.6\end{array}$ & & & & \\
\hline & & & Dx: Complex ankle injuries & & & & \\
\hline & & & Location: Australia & & & & \\
\hline
\end{tabular}

Table 4 displays the versatility of the FFI with all 3 domains and FFI Subscales and FFI-R uses across the studies. This shows that clinicians and researchers were choosing the FFI scales depending on the nature of their studies. Among the various scales of the FFI, we found the FFI with all 3 domains (full scale), the FFI pain subscale only, and a combination of the pain and disability subscales to be the most frequently used, whereas the FFI-R was the least frequently used. The Dutch adaptation of the FFI, the FFI5 pts, was mostly used in the Netherlands as an outcome measure in studies of many surgical interventions.
In summary, the FFI with all 3 domains, or as subscales, was frequently chosen as a measurement instrument across various studies and countries and among various age groups and sexes, for the assessment of acute and chronic foot and ankle conditions.

\section{Objective 2: Uses of the FFI and FFI-R in the field of foot health research}

The uses of the FFI and FFI-R are provided in detail in Tables 5, 6, 7, 8, 9, and 10. Table 12 describes the study types, the name of the instruments, and the first author's 
Table 8 Studies using foot function measures in orthotic intervention

\begin{tabular}{|c|c|c|c|c|c|c|c|}
\hline Instrument & $1^{\text {st }}$ Author & Study and objective & $\begin{array}{l}\text { Population } \\
\text { (N, Sex, Age, } \\
\text { Dx, location) }\end{array}$ & Methods \& Analyses & $\begin{array}{l}\text { Items/ } \\
\text { Domains/ } \\
\text { Subscales }\end{array}$ & $\begin{array}{l}\text { Measurement } \\
\text { scale }\end{array}$ & Summary evaluation \\
\hline \multirow[t]{9}{*}{$\overline{F F I, 1995}$} & $\begin{array}{l}\text { Budiman- } \\
\text { Mak, E [74] }\end{array}$ & $\begin{array}{l}\text { Outcome measure of } \\
\text { orthotic intervention } \\
\text { in hallux valgus } \\
\text { deformity }\end{array}$ & $\mathrm{N}=102$ & $\begin{array}{l}\text { RCT double blind Intent } \\
\text { to Treat Analysis FFI } \\
\text { applied at baseline and } \\
\text { each follow up visit }\end{array}$ & $\mathrm{FFI}$ & VAS & $\begin{array}{l}\text { This study suggest that } \\
\text { foot orthosis can } \\
\text { prevent or slowed the } \\
\text { progression of hallux } \\
\text { valgus deformity }\end{array}$ \\
\hline & & & $\begin{array}{l}\text { Treatment } \\
\text { group (N: 52) }\end{array}$ & & 23 items & & \\
\hline & & & $\begin{array}{l}\text { Mean age: } \\
60.2 \text { (SD 10.6) }\end{array}$ & & 3 domains & & \\
\hline & & & $\begin{array}{l}\text { Male: } 46 \\
(88.5 \%)\end{array}$ & & & & \\
\hline & & & $\begin{array}{l}\text { Control group } \\
\text { (N:50) }\end{array}$ & & & & \\
\hline & & & $\begin{array}{l}\text { Mean age: } \\
58.8 \text { (SD 11.9) }\end{array}$ & & & & \\
\hline & & & $\begin{array}{l}\text { Male: } 43 \\
(86 \%)\end{array}$ & & & & \\
\hline & & & DX:RA & & & & \\
\hline & & & Location: USA & & & & \\
\hline \multirow[t]{5}{*}{ FFI, 1996} & $\begin{array}{l}\text { Conrad, KJ } \\
{[70]}\end{array}$ & $\begin{array}{l}\text { Outcome measure- } \\
\text { Pain and function } \\
\text { measures }\end{array}$ & $N: 102$ & $\begin{array}{l}\text { RCT double blind Post } \\
\text {-test Random effect } \\
\text { model for longitudinal } \\
\text { data }\end{array}$ & $\mathrm{FFI}$ & VAS & $\begin{array}{l}\text { This study showed no } \\
\text { benefit on pain and } \\
\text { disability measures } \\
\text { between treatment } \\
\text { group and placebo } \\
\text { group }\end{array}$ \\
\hline & & & $\begin{array}{l}\text { Treatment } \\
\text { group (N: 52) } \\
\text { Mean age: } \\
60.2 \text { (SD 10) } \\
46 \text { male }\end{array}$ & $\begin{array}{l}\text { FFI applied at baseline } \\
\text { and at each follow up } \\
\text { visit }\end{array}$ & 23 items & & Conclusion: useful \\
\hline & & & $\begin{array}{l}\text { Control group } \\
\text { (N:50) Mean } \\
\text { age: } 58.8 \\
\text { (SD11.9) } 43 \\
\text { male }\end{array}$ & & 3 domains & & \\
\hline & & & Dx: RA & & & & \\
\hline & & & Location: USA & & & & \\
\hline \multirow[t]{5}{*}{ FFI, 1997} & $\begin{array}{l}\text { Caselli, MA } \\
{[77]}\end{array}$ & $\begin{array}{l}\text { Outcome measure - } \\
\text { Effectiveness of the } \\
\text { intervention }\end{array}$ & $\begin{array}{l}\text { N: } 34 ; \text { Mean } \\
\text { age: } 43 \\
(28-59) 12 \\
\text { male }\end{array}$ & $\begin{array}{l}\mathrm{RCT} \text {, not-blinded FFI } \\
\text { was applied at baseline } \\
\text { and at } 4 \text { weeks }\end{array}$ & $\mathrm{FFI}$ & $\begin{array}{l}\text { Categorical } \\
\text { rating scale }\end{array}$ & $\begin{array}{l}58 \%(11 / 19) \text { of } \\
\text { participants showed } \\
\text { improvement in pain } \\
\text { scores Conclusion: } \\
\text { useful }\end{array}$ \\
\hline & & & $\begin{array}{l}\text { Group 1: } \\
\text { Group with } \\
\text { magnet (N: 19) }\end{array}$ & & 23 items & & \\
\hline & & & $\begin{array}{l}\text { Group 2: } \\
\text { Group with } \\
\text { no magnet } \\
(\mathrm{N}: 15)\end{array}$ & & 3 domains & & \\
\hline & & & Dx: Heel pain & & & & \\
\hline & & & Location: USA & & & & \\
\hline $\mathrm{FFI}, 1997$ & $\begin{array}{l}\text { Caselli, MA } \\
\text { [68] }\end{array}$ & $\begin{array}{l}\text { Outcome measure - } \\
\text { Effectiveness of the } \\
\text { intervention }\end{array}$ & $\begin{array}{l}\text { N: } 35 \text {; Mean } \\
\text { age: } 42(23- \\
65) ; 18 \text { male }\end{array}$ & $\begin{array}{l}\text { RCT not blinded FFI } \\
\text { was applied at baseline } \\
\text { and at } 4 \text { weeks }\end{array}$ & $\mathrm{FFI}$ & $\begin{array}{l}\text { Categorical } \\
\text { rating scale }\end{array}$ & $\begin{array}{l}\text { FFI scores improved at } \\
4 \text { weeks reported as the } \\
\text { following: }\end{array}$ \\
\hline
\end{tabular}


Table 8 Studies using foot function measures in orthotic intervention (Continued)

\begin{tabular}{|c|c|c|c|c|c|c|c|}
\hline & & & \multicolumn{2}{|l|}{$\begin{array}{l}\text { Group 1: } \\
\text { Viscoped } \\
(\mathrm{N}: 16)\end{array}$} & \multicolumn{2}{|l|}{23 items } & 60\% (Group1) \\
\hline & & & \multicolumn{2}{|l|}{$\begin{array}{l}\text { Group 2: } \\
\text { Poron (N: 12) }\end{array}$} & \multicolumn{2}{|l|}{3 domains } & 43\% (Group 2) \\
\hline & & & \multicolumn{4}{|l|}{$\begin{array}{l}\text { Group 3: } \\
\text { Control(N: 7) }\end{array}$} & 10\% (Group 3) \\
\hline & & & \multicolumn{4}{|l|}{$\begin{array}{l}\text { Dx: Painful } \\
\text { submetatarsal } \\
\text { hyperkeratosis }\end{array}$} & Conclusion: useful \\
\hline & & & \multicolumn{4}{|l|}{ Location: USA } & \\
\hline \multirow[t]{8}{*}{ FFI, 1999} & $\begin{array}{l}\text { Pfeffer, G } \\
{[78]}\end{array}$ & $\begin{array}{l}\text { Outcome measure - } \\
\text { primary interest is in } \\
\text { pain subscale } \\
\text { outcome at } 8 \text { weeks }\end{array}$ & $\begin{array}{l}\text { N: } 236 ; \text { Mean } \\
\text { age: } 47 \\
\text { (23-81); } \\
160 \text { male }\end{array}$ & $\begin{array}{l}\text { RCT not blinded } 6 \\
\text { months interventions } \\
\text { multi-centers. FFI was } \\
\text { applied at baseline and } \\
\text { at } 8 \text { week intervals At } 8 \\
\text { weeks the group } \\
\text { response rate was } \\
88.2 \%\end{array}$ & $\mathrm{FFI}$ & $\begin{array}{l}\text { VAS rating } \\
\text { scale }\end{array}$ & $\begin{array}{l}\text { Pain subscale scores } \\
\text { improved at } 8 \text { weeks }\end{array}$ \\
\hline & & & \multicolumn{2}{|l|}{$\begin{array}{l}\text { Group 1: } \\
\text { Stretching } \\
\text { only (N: } 39) \\
\text { Mean age: } 47 \\
(25-81) 11 \\
\text { male }\end{array}$} & \multicolumn{2}{|l|}{23 items } & $\begin{array}{l}\text { Pain change scores } \\
\text { controlled for } \\
\text { covariates. Results are } \\
\text { reported as the } \\
\text { following: }\end{array}$ \\
\hline & & & \multicolumn{2}{|l|}{$\begin{array}{l}\text { Group 2: } \\
\text { Custom } \\
\text { orthoses \& } \\
\text { stretch (N: } 34) \\
\text { Mean age: } \\
48.5 \text { (23-69) } \\
11 \text { male }\end{array}$} & \multicolumn{2}{|l|}{3 domains } & Group 1: -17.2 \\
\hline & & & \multicolumn{2}{|l|}{$\begin{array}{l}\text { Group 3: } \\
\text { Silicon \& } \\
\text { stretch (N: } 51) \\
\text { Mean age: } \\
49.5 \text { (30-75) } \\
17 \text { male }\end{array}$} & & & Group 2: -16.9 \\
\hline & & & \multicolumn{2}{|l|}{$\begin{array}{l}\text { Group 4: } \\
\text { Rubber \& } \\
\text { stretch (N: 43) } \\
\text { Mean age: } 44 \\
\text { (27-69) } 11 \\
\text { male }\end{array}$} & & & Group 3: -23.9 \\
\hline & & & \multicolumn{2}{|l|}{$\begin{array}{l}\text { Group 5: Felt \& } \\
\text { stretch (N:42) } \\
\text { Mean age: } 48 \\
(26-76) 13 \\
\text { male }\end{array}$} & & & Group 4: -24.5 \\
\hline & & & \multicolumn{2}{|l|}{$\begin{array}{l}\text { Dx: Proximal } \\
\text { plantar } \\
\text { fasciitis }\end{array}$} & & & Group 5: -20.2 \\
\hline & & & \multicolumn{2}{|l|}{ Location: USA } & & & Conclusion: useful \\
\hline \multirow[t]{2}{*}{ FFI, 2001} & $\begin{array}{l}\text { Slattery, M } \\
\text { [82] }\end{array}$ & $\begin{array}{l}\text { Outcome measure - } \\
\text { effectiveness of the } \\
\text { intervention }\end{array}$ & $\begin{array}{l}\text { N: } 46 ; \text { Mean } \\
\text { age: } 24(6.2) \\
\text { Sex not } \\
\text { reported }\end{array}$ & $\begin{array}{l}\text { Observational } 6 \text { weeks } \\
\mathrm{FFI} \text { applied at baseline }\end{array}$ & $\mathrm{FFI}$ & $\begin{array}{l}\text { VAS rating } \\
\text { scale }\end{array}$ & $\begin{array}{l}\text { FFI scores of pain and } \\
\text { disability subscales } \\
\text { markedly improved at } 6 \\
\text { weeks }\end{array}$ \\
\hline & & & \multicolumn{2}{|l|}{$\begin{array}{l}\text { Dx: } \\
\text { Hemophilic } \\
\text { foot and ankle }\end{array}$} & \multicolumn{2}{|l|}{ 23items } & Conclusion: useful \\
\hline
\end{tabular}


Table 8 Studies using foot function measures in orthotic intervention (Continued)

\begin{tabular}{|c|c|c|c|c|c|c|c|}
\hline & & & $\begin{array}{l}\text { arthropathy at } \\
\text { level } 1-5 \text { joint } \\
\text { damange }\end{array}$ & & & & \\
\hline & & & $\begin{array}{l}\text { Location: } \\
\text { Australia }\end{array}$ & & 3 domains & & \\
\hline \multirow[t]{5}{*}{ FFI, 2002} & $\begin{array}{l}\text { Gross, MT } \\
{[79]}\end{array}$ & $\begin{array}{l}\text { Outcome measure - } \\
\text { Effectiveness of the } \\
\text { intervention } \\
\text { correlation with } 100 \\
\text { meter walk and VAS } \\
\text { pain scale }\end{array}$ & N: 15; 8 male & $\begin{array}{l}\text { Pre-post test design FFI } \\
\text { was applied at baseline } \\
\text { and post orthosis at } \\
12-17 \text { days }\end{array}$ & $\begin{array}{l}\text { FFI } 18 \\
\text { items Pain } \\
\text { and } \\
\text { disability } \\
\text { scales }\end{array}$ & $\begin{array}{l}\text { VAS rating } \\
\text { scale }\end{array}$ & $\begin{array}{l}\text { Pain and disability } \\
\text { improved. The author } \\
\text { suggested to modify } \\
\text { FFI items if FFI will be } \\
\text { used for plantar fasciitis. }\end{array}$ \\
\hline & & & $\begin{array}{l}\text { Mean age } \\
\text { male: } 43.8 \\
(\mathrm{SD}=6.3)\end{array}$ & & & & Conclusion: useful \\
\hline & & & $\begin{array}{l}\text { Mean age } \\
\text { female: } 45.9 \\
(\mathrm{SD}=11.9)\end{array}$ & & & & \\
\hline & & & $\begin{array}{l}\text { Dx: Plantar } \\
\text { fasciitis }\end{array}$ & & & & \\
\hline & & & Location: USA & & & & \\
\hline \multirow[t]{5}{*}{ FFI, 2002} & $\begin{array}{l}\text { Woodburn, J } \\
{[80]}\end{array}$ & $\begin{array}{l}\text { Outcome measure - } \\
\text { effectiveness of the } \\
\text { intervention }\end{array}$ & $\begin{array}{l}\text { N: 98; } \\
\text { Orthosis/ } \\
\text { vsControl }\end{array}$ & $\begin{array}{l}\text { RCT double blind; } 30 \\
\text { months study. FFI was } \\
\text { applied at } 3,6,12,18 \text {, } \\
24 \text {, and } 30 \text { months }\end{array}$ & $\mathrm{FFI}$ & $\begin{array}{l}\text { VAS rating } \\
\text { scale }\end{array}$ & $\begin{array}{l}\text { FFI scores improved at } \\
\text { the completion of the } \\
\text { RCT }\end{array}$ \\
\hline & & & $\begin{array}{l}\text { Orthosis } \\
\text { (N: } 50) \text { Mean } \\
\text { age: } 54 \\
(\mathrm{SD}=11.8) 16 \\
\text { male }\end{array}$ & & 23 items & & Conclusion: useful \\
\hline & & & $\begin{array}{l}\text { Control (N: 48) } \\
\text { Mean age: } 53 \\
(\mathrm{SD}=11.1) 17 \\
\text { male }\end{array}$ & & 3 domains & & \\
\hline & & & $\begin{array}{l}\text { Dx: RA rear } \\
\text { foot valgus } \\
\text { deformity }\end{array}$ & & & & \\
\hline & & & Location: UK & & & & \\
\hline \multirow[t]{5}{*}{ FFI, 2005} & $\begin{array}{l}\text { Powell, M } \\
\text { [83] }\end{array}$ & $\begin{array}{l}\text { Outcome measure - } \\
\text { Validation of The } \\
\text { Pediatric Pain VAS } \\
\text { Questionnaires, } \\
\text { Pediatric quality of life } \\
\text { (PedQOL) inventory } \\
\text { physical function scale }\end{array}$ & $\begin{array}{l}\mathrm{N}: 40 ; \text { Custom } \\
\text { orthoses: N: } 15 \text {; } \\
2 \text { Male Mean } \\
\text { age: } 12.14\end{array}$ & $\begin{array}{l}\text { RCT } 3 \text { arms, Single } \\
\text { blinded }\end{array}$ & $\mathrm{FFI}$ & $\begin{array}{l}\text { VAS rating } \\
\text { scale }\end{array}$ & $\begin{array}{l}\text { The largest } \\
\text { improvement of FFI } \\
\text { scores was in the } \\
\text { custom orthoses. VAS } \\
\text { scoring appears } \\
\text { applicable in children }\end{array}$ \\
\hline & & & $\begin{array}{l}\text { Insert N: 12; } 4 \\
\text { Male Mean } \\
\text { age: } 12.7\end{array}$ & $\begin{array}{l}\text { Intent to Treat Analysis; } \\
\text { ANOVA }\end{array}$ & 23 items & & Conclusion: useful \\
\hline & & & $\begin{array}{l}\text { Athletic shoes } \\
\text { N: } 13 ; 4 \text { Male } \\
\text { Mean age: } \\
13.77\end{array}$ & $\begin{array}{l}\text { FFI was applied at } \\
\text { baseline and at } 3 \\
\text { months }\end{array}$ & 3 domains & & \\
\hline & & & $\begin{array}{l}\text { Dx: JRA and } \\
\text { foot pain }\end{array}$ & & & & \\
\hline & & & Location: USA & & & & \\
\hline FFI, 2006 & $\begin{array}{l}\text { Magalahaes, } \\
\text { E [69] }\end{array}$ & $\begin{array}{l}\text { Outcome measure - } \\
\text { Concurrent measure } \\
\text { with Health }\end{array}$ & N: 36; 5 Male & $\begin{array}{l}\text { Prospective } \\
\text { observational }\end{array}$ & $\mathrm{FFI}$ & $\begin{array}{l}\text { VAS rating } \\
\text { scale }\end{array}$ & $\begin{array}{l}\text { FFI scores in pain, } \\
\text { disability, activity } \\
\text { limitation improved; no }\end{array}$ \\
\hline
\end{tabular}


Table 8 Studies using foot function measures in orthotic intervention (Continued)

\begin{tabular}{|c|c|c|c|c|c|c|c|}
\hline & & $\begin{array}{l}\text { Assessment } \\
\text { Questionnaires (HAQ) }\end{array}$ & & & & & $\begin{array}{l}\text { correlations with } \mathrm{HAQ} \\
\text { scores }\end{array}$ \\
\hline & & & Orthosis N: 28 & $\begin{array}{l}2 \text { treatment groups; } 6 \\
\text { months trial }\end{array}$ & \multicolumn{2}{|l|}{23 items } & Conclusion: useful \\
\hline & & & Sham N: 8 & $\begin{array}{l}\text { FFI was applied at } \\
\text { baseline, 30, 90, and } \\
180 \text { days }\end{array}$ & \multicolumn{2}{|l|}{3 domains } & \\
\hline & & & $\begin{array}{l}\text { Mean age: } 46 \\
(32-68) \text { RA } \\
\text { years } 11 \\
(1-34)\end{array}$ & & & & \\
\hline & & & $\begin{array}{l}\text { Location: } \\
\text { Brazil }\end{array}$ & & & & \\
\hline \multirow[t]{6}{*}{ FFI, 2007} & \multirow[t]{6}{*}{$\begin{array}{l}\text { Williams, AE } \\
\text { [71] }\end{array}$} & \multirow[t]{6}{*}{$\begin{array}{l}\text { Outcome measure - } \\
\text { Concurrent measure } \\
\text { with FHSQ for } \\
\text { designed shoes } \\
\text { intervention }\end{array}$} & $\begin{array}{l}\mathrm{N}: 80 ; 35 \\
\text { maleAge: N/A }\end{array}$ & \multirow[t]{6}{*}{$\begin{array}{l}\text { RCT single blinded; } 12 \\
\text { weeks trial. FFI was } \\
\text { applied at baseline and } \\
12 \text { weeks N: } 34 \\
\text { completed the study }\end{array}$} & $\mathrm{FFI}$ & \multirow[t]{6}{*}{$\begin{array}{l}\text { VAS rating } \\
\text { scale }\end{array}$} & $\begin{array}{l}\text { Both scores of FFI and } \\
\text { FHSQ were improved at } \\
12 \text { weeks }\end{array}$ \\
\hline & & & $\begin{array}{l}\text { Group 1: } \\
\text { Designed } \\
\text { shoes (N: 40); } \\
11 \text { male }\end{array}$ & & 23 items & & $\begin{array}{l}\text { Between groups } \\
\text { general health was } \\
\text { unchanged }\end{array}$ \\
\hline & & & $\begin{array}{l}\text { Group 2: } \\
\text { Traditional } \\
\text { shoes (N: 40) } \\
19 \text { male }\end{array}$ & & \multirow[t]{4}{*}{3 domains } & & Conclusion: useful \\
\hline & & & $\begin{array}{l}\text { RA } 17 \text { years } \\
\text { (14.4 yrs) }\end{array}$ & & & & \\
\hline & & & $\begin{array}{l}\text { Dx: Hallux } \\
\text { valgus }\end{array}$ & & & & \\
\hline & & & Location: UK & & & & \\
\hline \multirow[t]{3}{*}{ FFI, 2008} & \multirow[t]{3}{*}{ Lin, JL [81] } & \multirow[t]{3}{*}{$\begin{array}{l}\text { Outcome measure - } \\
\text { Validation with AOFAS } \\
\text { VAS pain scale SF-36 }\end{array}$} & N: 32; 6 male & \multirow[t]{3}{*}{$\begin{array}{l}\text { Observational } 7-10 \\
\text { years (mean } 8.8 \text { years); } \\
\text { FFI was applied at the } \\
\text { end of the observation }\end{array}$} & & & $\begin{array}{l}\text { FFI scores for pain and } \\
\text { disability were } \\
\text { improved and well } \\
\text { correlated with AOFAS } \\
\text { scores }\end{array}$ \\
\hline & & & $\begin{array}{l}\text { Dx: Stage II } \\
\text { posterior } \\
\text { tibialis tendon } \\
\text { dysfunction } \\
\text { (PTTD) }\end{array}$ & & & & Conclusion: useful \\
\hline & & & Location: USA & & & & \\
\hline \multirow[t]{5}{*}{$\mathrm{FFI}, 2009$} & \multirow[t]{5}{*}{ Cho, NS [72] } & \multirow[t]{5}{*}{$\begin{array}{l}\text { Outcome measure - } \\
\text { Validation with VAS } \\
\text { pain scale }\end{array}$} & $\begin{array}{l}\mathrm{N}: 42 ; \text { Semi- } \\
\text { rigid insole: } \\
\mathrm{N}: 22 \\
0 \text { male }\end{array}$ & \multirow[t]{5}{*}{$\begin{array}{l}\text { RCT single blinded } 6 \\
\text { month trial FFI was } \\
\text { applied at baseline and } \\
6 \text { month At } 6 \text { months } \\
\text { N34 completed the } \\
\text { study }\end{array}$} & $\mathrm{FFI}$ & \multirow[t]{5}{*}{$\begin{array}{l}\text { VAS rating } \\
\text { scale }\end{array}$} & $\begin{array}{l}\text { Semi-rigid insole group } \\
\text { showed markedly } \\
\text { improved FFI scores }\end{array}$ \\
\hline & & & $\begin{array}{l}11 \text { fore foot/11 } \\
\text { hind foot }\end{array}$ & & 23 items & & Conclusion: useful \\
\hline & & & $\begin{array}{l}\text { Mean age: } \\
48.7(\mathrm{SD}=11.6)\end{array}$ & & \multirow[t]{3}{*}{3 domains } & & \\
\hline & & & $\begin{array}{l}\text { Soft insole: } \\
\text { N: } 20 ; 0 \text { male } \\
11 \text { fore/10 } \\
\text { hind foot }\end{array}$ & & & & \\
\hline & & & $\begin{array}{l}\text { Mean age: } \\
48.7(\mathrm{SD}=11.7)\end{array}$ & & & & \\
\hline
\end{tabular}


Table 8 Studies using foot function measures in orthotic intervention (Continued)

\begin{tabular}{|c|c|c|c|c|c|c|c|}
\hline & & & $\begin{array}{l}\text { Dx: RA foot } \\
\text { deformity, } \\
\text { hind or } \\
\text { forefoot }\end{array}$ & & & & \\
\hline & & & $\begin{array}{l}\text { Location: } \\
\text { Korea }\end{array}$ & & & & \\
\hline \multirow[t]{5}{*}{ FFI, 2009} & $\begin{array}{l}\text { Novak, P } \\
{[84]}\end{array}$ & $\begin{array}{l}\text { Outcome measure - } \\
\text { Correlation with } 6 \\
\text { minute walk time }\end{array}$ & $\begin{array}{l}\text { N: } 40 ; \text { Mean } \\
\text { age: } 56.23 ; 2 \\
\text { male }\end{array}$ & $\begin{array}{l}\text { RCT double blinded } 6 \\
\text { months trial FFI was } \\
\text { applied at baseline } \\
\text { visits } 1,2 \text {, and } 3 \text { at } 6 \\
\text { months }\end{array}$ & $\mathrm{FFI}$ & $\begin{array}{l}\text { VAS rating } \\
\text { scale }\end{array}$ & $\begin{array}{l}\text { Pain improved } \\
\text { correlation with } 6 \\
\text { minute walk time was } \\
\text { moderate }\end{array}$ \\
\hline & & & $\begin{array}{l}\text { Orthosis } \\
\text { (N: 20) Mean } \\
\text { age: } 55.7 \\
(\mathrm{SD}=9.31) \mathrm{RA}: \\
10.5 \mathrm{yrs} \\
(\mathrm{SD}=8.17)\end{array}$ & & 9 items & & Conclusion: useful \\
\hline & & & $\begin{array}{l}\text { Control }(\mathrm{N}: 20) \\
\text { Mean age: } \\
56.75 \\
(\mathrm{SD}=11.1) \mathrm{RA}: \\
11.5 \mathrm{yrs} \\
(\mathrm{SD}=6.86)\end{array}$ & & Pain scale & & \\
\hline & & & Dx: RA & & & & \\
\hline & & & $\begin{array}{l}\text { Location: } \\
\text { Slovenia }\end{array}$ & & & & \\
\hline \multirow[t]{7}{*}{ FFI, 2009} & $\begin{array}{l}\text { Baldassin, V } \\
\text { [35] }\end{array}$ & $\begin{array}{l}\text { Outcome measure - } \\
\text { pain relief }\end{array}$ & $\begin{array}{l}\text { N: } 142 ; \\
\text { Custom } \\
\text { Orthosis: } \\
\mathrm{N}=72\end{array}$ & $\begin{array}{l}\text { RCT double blind; } 8 \\
\text { weeks trial. FFI was } \\
\text { applied at } 4 \text { and } 8 \\
\text { weeks }\end{array}$ & $\mathrm{FFI}$ & $\begin{array}{l}\text { VAS rating } \\
\text { scale }\end{array}$ & $\begin{array}{l}\text { Less pain was observed } \\
\text { in both groups but no } \\
\text { significant differences } \\
\text { between groups }\end{array}$ \\
\hline & & & $\begin{array}{l}\text { Mean age: } \\
55.7(\mathrm{SD}=12.4)\end{array}$ & & 23 items & & Conclusion: useful \\
\hline & & & $\begin{array}{l}\text { RA: } 47.2 \text { yrs } \\
(S D=8.17) 51 \\
\text { male }\end{array}$ & & 3 domains & & \\
\hline & & & $\begin{array}{l}\text { Prefabricated } \\
\text { orthosis: } \mathrm{N}=70\end{array}$ & & $\begin{array}{l}\text { Pain } \\
\text { subscales } \\
9 \text { items } \\
\text { (modified) }\end{array}$ & & \\
\hline & & & $\begin{array}{l}\text { Mean age: } \\
47.5(\mathrm{SD}=11.5)\end{array}$ & & & & \\
\hline & & & $\begin{array}{l}\text { Dx: Plantar } \\
\text { fasciitis }\end{array}$ & & & & \\
\hline & & & $\begin{array}{l}\text { Location: } \\
\text { Brazil }\end{array}$ & & & & \\
\hline \multirow[t]{5}{*}{ FFI-R, 2009} & Rao, S [75] & $\begin{array}{l}\text { Outcome measure - } \\
\text { FFI-R scores translated } \\
\text { to clinical measure } \\
\text { MDC }_{90} \text {, Correlation } \\
\text { with medial mid-foot } \\
\text { pressure loading }\end{array}$ & N: 20; 0 male & $\begin{array}{l}\text { Intervention } 4 \text { weeks } \\
\text { FFI-R was applied at pre } \\
\text { and post intervention } \\
\text { Statistician was blinded } \\
\text { from data sources }\end{array}$ & FFI-R & Likert & $\begin{array}{l}\text { Total FFI-R scores } \\
\text { improved correlated } \\
\text { with significant } \\
\text { reduction in pressure } \\
\text { loading of the medial } \\
\text { aspect of the midfoot }\end{array}$ \\
\hline & & & $\begin{array}{l}\text { Mean age: } 63 \\
(55-78)\end{array}$ & & 68 items & & Conclusion: useful \\
\hline & & & $\begin{array}{l}\text { Full length } \\
\text { orthosis }\end{array}$ & & Long form & & \\
\hline & & & $\begin{array}{l}\text { Dx: Midfoot } \\
\text { arthritis }\end{array}$ & & & & \\
\hline & & & Location: USA & & & & \\
\hline
\end{tabular}


Table 8 Studies using foot function measures in orthotic intervention (Continued)

\begin{tabular}{|c|c|c|c|c|c|c|c|}
\hline \multirow[t]{5}{*}{ FFI-R, 2010} & Rao, S [76] & $\begin{array}{l}\text { Outcome measure - } \\
\text { Clinical measure MDC } \\
90 \text { validation with } \\
\text { segmental foot } \\
\text { kinematic values }\end{array}$ & $\mathrm{N}: 30 ; 2$ male & $\begin{array}{l}\text { Intervention } 4 \text { weeks } \\
\text { FFI-R was applied at pre } \\
\text { and post intervention }\end{array}$ & $\mathrm{FFI}-\mathrm{R}$ & Likert & $\begin{array}{l}\text { Full length foot } \\
\text { orthoses reduced } \\
\text { motion of the } 1^{\text {st }} \\
\text { metatarsophalangeal } \\
\text { and was significantly } \\
\text { correlated with FFI-R } \\
\text { scores }\end{array}$ \\
\hline & & & $\begin{array}{l}\text { Mean age: } 62 \\
(47-78)\end{array}$ & & 68 items & & Conclusion: useful \\
\hline & & & $\begin{array}{l}\text { Full length } \\
\text { carbon } \\
\text { graphite } \\
\text { orthosis }\end{array}$ & & Long form & & \\
\hline & & & $\begin{array}{l}\text { Dx: Midfoot } \\
\text { arthritis }\end{array}$ & & & & \\
\hline & & & Location: USA & & & & \\
\hline \multirow[t]{5}{*}{ FFI, 2010} & $\begin{array}{l}\text { Welsh, BJ } \\
{[73]}\end{array}$ & $\begin{array}{l}\text { Outcome measure - } \\
\text { validation with foot } \\
\text { kinematic values VAS } \\
\text { pain scale }\end{array}$ & $\mathrm{N}: 32 ; 6$ male & $\begin{array}{l}\text { Case series } 24 \text { weeks } \\
\text { Pre-post test design }\end{array}$ & $\mathrm{FFI}$ & $\begin{array}{l}\text { VAS rating } \\
\text { scale }\end{array}$ & $\begin{array}{l}\text { FFI pain subscale } \\
\text { significantly improved } \\
\text { and met the criteria of } \\
\text { equivalence to } \\
\text { analgesic response. This } \\
\text { pain reduction was not } \\
\text { correlated with that of } \\
\text { the biomechanical } \\
\text { changes of the } 1^{\text {st }} \\
\text { metatarsophalangeal } \\
\text { joint. }\end{array}$ \\
\hline & & & $\begin{array}{l}\text { Mean age: } 42 \\
(\mathrm{SD}=11.5)\end{array}$ & & 9 items & & Conclusion: useful \\
\hline & & & $\begin{array}{l}\text { Pre-fabricated } \\
\text { vs. custom } \\
\text { orthosis }\end{array}$ & & $\begin{array}{l}\text { Pain } \\
\text { subscale }\end{array}$ & & \\
\hline & & & $\begin{array}{l}\text { Dx: MTP joint } \\
\text { pain }\end{array}$ & & & & \\
\hline & & & Location: UK & & & & \\
\hline \multirow[t]{4}{*}{$\mathrm{FFI}, 2010$} & Clark H [85] & $\begin{array}{l}\text { Outcome measure - } \\
\text { Orthosis reduced pain } \\
\text { and disability and } \\
\text { correlated with gait } \\
\text { parameter }\end{array}$ & $\begin{array}{l}\text { N: } 41 \text {; Gender } \\
\text { not reported }\end{array}$ & $\begin{array}{l}\text { RCT single blind } 16 \\
\text { weeks trial. FFI was } \\
\text { applied at baseline, } 8 \\
\text { and } 16 \text { weeks }\end{array}$ & $\mathrm{FFI}$ & $\begin{array}{l}\text { VAS rating } \\
\text { scale }\end{array}$ & $\begin{array}{l}\text { FFI scores were } \\
\text { improved in orthoses } \\
\text { and simple insole } \\
\text { groups but the } \\
\text { intervention did not } \\
\text { improve gait parameter. }\end{array}$ \\
\hline & & & $\begin{array}{l}\text { Orthosis: N: } \\
\text { 20; Simple } \\
\text { insole: N: } 21\end{array}$ & & 23 items & & Conclusion: useful \\
\hline & & & $\begin{array}{l}\text { Age }>18 \text { years; } \\
\text { RA }>3 \text { years }\end{array}$ & & 3 domains & & \\
\hline & & & $\begin{array}{l}\text { Location: New } \\
\text { Zealand }\end{array}$ & & & & \\
\hline
\end{tabular}

name and the reference number. The studies are grouped by how the instruments were used and ordered chronologically within group.

\section{Measurement, validation and cultural adaptation}

Table 12 describes the utility of the FFI and FFI-R in studies of foot function measures and includes 17 articles. Category A New Instruments. Includes four articles in which foot health measures are described including the original FFI [7], the FFI-R [11]. The FFI Side to Side was derived from pain and disability subscales of the FFI [23]. The Ankle Osteoarthritis Scale (AOS) [24]; measured foot problems related to foot and ankle osteoarthritis. Agel et al. [25] modified the rating scale of the FFI pain and function subscales from the visual analog rating scale (VAS) to the Likert categorical scale; this modification was tested in a sample of individuals with nontraumatic foot complaints, and the metric of the Likert 
Table 9 Studies using foot function measures in various interventions

\begin{tabular}{|c|c|c|c|c|c|c|c|}
\hline Instrument & $\begin{array}{l}1^{\text {st }} \\
\text { Author }\end{array}$ & Objective & $\begin{array}{l}\text { Population } \\
\text { (N, Sex, } \\
\text { Age, Dx, } \\
\text { location) }\end{array}$ & Analysis & $\begin{array}{l}\text { Items/ } \\
\text { domains/ } \\
\text { subscales }\end{array}$ & $\begin{array}{l}\text { Response } \\
\text { type }\end{array}$ & Summary evaluation \\
\hline \multirow[t]{3}{*}{$\begin{array}{l}\text { Foot } \\
\text { Function } \\
\text { Index, } 2005\end{array}$} & \multirow[t]{3}{*}{$\begin{array}{l}\text { Cui, Q } \\
{[86]}\end{array}$} & $\begin{array}{l}\text { Improvement in pain } \\
\text { and function }\end{array}$ & $\begin{array}{l}\text { N: } 5 \text {; Mean } \\
\text { age: } 40 \\
\text { (range: } 25- \\
54) ; 3 \text { male }\end{array}$ & \multirow[t]{3}{*}{$\begin{array}{l}\text { Retrospective study; Follow-up } 24 \\
\text { months ( } 16-30 \text { months). FFI was } \\
\text { applied at pre and at post treatment }\end{array}$} & $\mathrm{FFI}$ & \multirow[t]{3}{*}{ VAS } & $\begin{array}{l}\text { FFI scores improved } \\
\text { on } 3 \text { out of } 5 \text { patients } \\
\text { post surgery. }\end{array}$ \\
\hline & & \multirow[t]{2}{*}{$\begin{array}{l}\text { Cortisone injection } \\
\text { and arthroscopic } \\
\text { surgery }\end{array}$} & $\begin{array}{l}\text { Dx: Post } \\
\text { traumatic } \\
\text { ankle } \\
\text { adhesive } \\
\text { capsulitis }\end{array}$ & & $\begin{array}{l}\text { Pain and } \\
\text { disability } \\
\text { subscales }\end{array}$ & & \multirow[t]{2}{*}{ Conclusion: useful } \\
\hline & & & $\begin{array}{l}\text { Location: } \\
\text { USA }\end{array}$ & & 18 items & & \\
\hline \multirow[t]{6}{*}{$\begin{array}{l}\text { Foot } \\
\text { Function } \\
\text { Index, } 2005\end{array}$} & \multirow[t]{6}{*}{$\begin{array}{l}\text { Di } \\
\text { Giovanni, } \\
\text { BF [87] }\end{array}$} & \multirow[t]{6}{*}{$\begin{array}{l}\text { Reduction of foot pain } \\
\text { Stretching exercise } \\
\text { and wearing foot } \\
\text { insert }\end{array}$} & $\begin{array}{l}\mathrm{N}: 101 ; 33 \\
\text { male }\end{array}$ & \multirow{6}{*}{$\begin{array}{l}\text { Randomized clinical Trial } \\
\text { Longitudinal mixed-model analysis } \\
\text { of covariance FFI was applied at } \\
\text { baseline and at } 8 \text { weeks }(N=: 82 \text {, } \\
A=46, B=36) \text {. At } 2 \text { years }(N:=66 \text {, } \\
A=39, B=27)\end{array}$} & $\mathrm{FFI}$ & \multirow[t]{6}{*}{ VAS } & $\begin{array}{l}\text { FFI pain scores } \\
\text { improved at } 2 \text { weeks } \\
\text { and much improved } \\
\text { at } 2 \text { years }\end{array}$ \\
\hline & & & $\begin{array}{l}\text { Mean age: } \\
45 \text { (range } \\
23-60)\end{array}$ & & $\begin{array}{l}\text { Pain } \\
\text { subscale }\end{array}$ & & $\begin{array}{l}\text { Group A had a better } \\
\text { scores than B }\end{array}$ \\
\hline & & & $\begin{array}{l}\text { Group A: } \\
\text { Plantar } \\
\text { fascia } \\
\text { stretching }\end{array}$ & & 9 items & & \multirow[t]{4}{*}{ Conclusion: useful } \\
\hline & & & $\begin{array}{l}\text { Group B: } \\
\text { Achillus } \\
\text { tendon } \\
\text { stretching }\end{array}$ & & & & \\
\hline & & & $\begin{array}{l}\text { DX: Plantar } \\
\text { fasciitis }\end{array}$ & & & & \\
\hline & & & $\begin{array}{l}\text { Location: } \\
\text { USA }\end{array}$ & & & & \\
\hline \multirow[t]{4}{*}{$\begin{array}{l}\text { Foot } \\
\text { Function } \\
\text { Index, } 2009\end{array}$} & \multirow[t]{4}{*}{$\begin{array}{l}\text { Kulig,K } \\
{[88]}\end{array}$} & \multirow[t]{4}{*}{$\begin{array}{l}\text { Validation of physical } \\
\text { activity scale (PAS) } \\
\text { and } 5 \text { minutes walk } \\
\text { test, and simple heel } \\
\text { raise test. }\end{array}$} & $\begin{array}{l}\mathrm{N}=: 10 ; \\
\text { Gender: NA }\end{array}$ & \multirow[t]{4}{*}{$\begin{array}{l}\text { Exercise intervention: } 10 \text { weeks } \\
\text { Follow up: } 6 \text { months. FFI was } \\
\text { applied at baseline, } 10 \text { weeks and } 6 \\
\text { months }\end{array}$} & $\mathrm{FFI}$ & \multirow[t]{4}{*}{ VAS } & $\begin{array}{l}\text { FFI pain and function } \\
\text { subscales were used } \\
\text { to monitor pre- and } \\
\text { post-intervention } \\
\text { outcomes. }\end{array}$ \\
\hline & & & $\begin{array}{l}\text { Mean } \\
\text { age:52.1 } \\
\text { (SD6.5) }\end{array}$ & & 23 items & & \multirow[t]{3}{*}{ Conclusion: useful } \\
\hline & & & $\begin{array}{l}\text { DX: } \\
\text { Posterior } \\
\text { tibial } \\
\text { tendon } \\
\text { dysfunction }\end{array}$ & & 3 domains & & \\
\hline & & & $\begin{array}{l}\text { Location: } \\
\text { USA }\end{array}$ & & & & \\
\hline \multirow[t]{4}{*}{$\begin{array}{l}\text { Foot } \\
\text { Function } \\
\text { Index, } 2010\end{array}$} & \multirow[t]{4}{*}{$\begin{array}{l}\text { Rompe, } \\
\text { JD [89] }\end{array}$} & \multirow{4}{*}{$\begin{array}{l}\text { Outcomes: Change } \\
\text { scores between } \\
\text { observations. } \\
\text { Stretching and shock } \\
\text { wave therapy }\end{array}$} & $\begin{array}{l}\mathrm{N}=54 ; 18 \\
\text { male }\end{array}$ & \multirow{4}{*}{$\begin{array}{l}\text { Randomized parallel treatment } 15 \\
\text { months trial. Intend to treat analysis } \\
\text { FFI was applied at baseline, } 4 \text { and } \\
15 \text { months }\end{array}$} & $\mathrm{FFI}$ & \multirow[t]{4}{*}{ VAS } & $\begin{array}{l}\text { FFl pain scores were } \\
\text { better in stretching } \\
\text { exercise group }\end{array}$ \\
\hline & & & $\begin{array}{l}\text { Mean age: } \\
53.1 \\
(\mathrm{SD}=27.7)\end{array}$ & & $\begin{array}{l}\text { Pain } \\
\text { subscale }\end{array}$ & & \multirow[t]{3}{*}{ Conclusion: useful } \\
\hline & & & $\begin{array}{l}\text { Dx: Plantar } \\
\text { Fasciotomy }\end{array}$ & & 9 items & & \\
\hline & & & $\begin{array}{l}\text { Location: } \\
\text { Germany }\end{array}$ & & & & \\
\hline
\end{tabular}


Table 10 Studies using foot function measures in observational studies

\begin{tabular}{|c|c|c|c|c|c|c|c|}
\hline Instrument & $1^{\text {st }}$ Author & Study and objective & $\begin{array}{l}\text { Population } \\
\text { (N, Sex, Age, } \\
\text { Dx, location) }\end{array}$ & $\begin{array}{l}\text { methods \& } \\
\text { analyses }\end{array}$ & $\begin{array}{l}\text { Items/ } \\
\text { domains/ } \\
\text { subscales }\end{array}$ & $\begin{array}{l}\text { Response } \\
\text { type }\end{array}$ & Summary evaluation \\
\hline \multirow[t]{10}{*}{ FFI, 2004} & \multirow[t]{10}{*}{ Novak, P [4] } & $\begin{array}{l}\text { Epidemiology of Type II } \\
\text { Diabetes Mellitus }\end{array}$ & & & & & \\
\hline & & \multirow{9}{*}{$\begin{array}{l}\text { Correlation of pain score } \\
\text { with } 6 \text { minute walk time; } \\
\text { Comparing intergroup } \\
\text { pain score }\end{array}$} & \multirow[t]{2}{*}{$\begin{array}{l}\text { Total N: 90; } 3 \\
\text { groups; }\end{array}$} & $\begin{array}{l}\text { Cross-Sectional } \\
\text { study }\end{array}$ & $\mathrm{FFI}$ & \multirow[t]{9}{*}{ VAS scale } & \multirow{2}{*}{$\begin{array}{l}\text { High pain score correlated with } \\
\text { shorter distance walk, group with } \\
\text { Type II diabetes neuropathy with } \\
\text { symptoms showed the highest } \\
\text { pain scores }\end{array}$} \\
\hline & & & & $\begin{array}{l}\text { Descriptive \& } \\
\text { correlation } \\
\text { statistics }\end{array}$ & & & \\
\hline & & & $\begin{array}{l}\text { Neuropathy } \\
\text { with } \\
\text { symptoms } \\
\text { N: } 30 \text { Mean } \\
\text { age } 64.87 \\
(\mathrm{SD}=11)\end{array}$ & & 9 items & & \multirow[t]{7}{*}{ Conclusion: useful } \\
\hline & & & 20 male & & Pain scale & & \\
\hline & & & $\begin{array}{l}\text { Neuropathy, } \\
\text { no symptoms } \\
\text { N:30; Mean } \\
\text { age: } 64.87 \\
\text { (SD }=11 \text { ) }\end{array}$ & & & & \\
\hline & & & 20 male; & & & & \\
\hline & & & $\begin{array}{l}\text { Healthy } \\
\text { volunteers } \\
\text { N: } 30 ; \text { Mean } \\
\text { age: } 64.87 \\
(\mathrm{SD}=11)\end{array}$ & & & & \\
\hline & & & 20 male; & & & & \\
\hline & & & Slovenia & & & & \\
\hline \multirow[t]{5}{*}{ FFI, 2004} & \multirow[t]{5}{*}{$\begin{array}{l}\text { Williams, AE } \\
\text { [90] }\end{array}$} & \multirow[t]{2}{*}{$\begin{array}{l}\text { Epidemiology Rheumatic } \\
\text { diseases }\end{array}$} & \multirow[t]{2}{*}{$\begin{array}{l}\mathrm{N}: 139 ; 39 \\
\text { male }\end{array}$} & $\begin{array}{l}\text { Cross sectional } \\
\text { study }\end{array}$ & $\mathrm{FFI}$ & \multirow[t]{5}{*}{ VAS scale } & \multirow{2}{*}{$\begin{array}{l}\text { FFI scores showed a high } \\
\text { prevalence of foot and ankle } \\
\text { pathologies, which indicated the } \\
\text { need of podiatry care }\end{array}$} \\
\hline & & & & $\begin{array}{l}\text { Descriptive } \\
\text { statistics }\end{array}$ & & & \\
\hline & & $\begin{array}{l}\text { To assess foot health } \\
\text { status }\end{array}$ & Age: NA & & 23 items & & Conclusion: useful \\
\hline & & & $\begin{array}{l}\text { Inflammatory } \\
\text { and } \\
\text { degenerative } \\
\text { joint diseases }\end{array}$ & & 3 domains & & \\
\hline & & & UK & & & & \\
\hline \multirow[t]{3}{*}{ FFI, 2006} & \multirow[t]{3}{*}{$\begin{array}{l}\text { Williams, AE } \\
\text { [91] }\end{array}$} & $\begin{array}{l}\text { Epidemiology of Paget } \\
\text { diseases of the foot } \\
\text { Concurrent measures of } \\
\text { FSI and quality of Life 12- } \\
\text { items short form }\end{array}$ & $\begin{array}{l}\text { N: } 134 ; 64 \\
\text { male }\end{array}$ & $\begin{array}{l}\text { Cross sectional } \\
\text { study } \\
\text { Descriptive } \\
\text { statistics }\end{array}$ & $\mathrm{FFI}$ & \multirow[t]{3}{*}{ VAS scale } & $\begin{array}{l}\text { Correlations of scores were not } \\
\text { found between instruments }\end{array}$ \\
\hline & & & $\begin{array}{l}\text { Mean age: } \\
74.5(46-91)\end{array}$ & & 23 items & & \multirow[t]{2}{*}{ Conclusion: not useful } \\
\hline & & & UK & & 3 domains & & \\
\hline \multirow[t]{2}{*}{$\mathrm{FFI}, 2006$} & \multirow[t]{2}{*}{$\begin{array}{l}\text { Rosenbaum, } \\
\text { D [95] }\end{array}$} & $\begin{array}{l}\text { Plantar sensitivity } \\
\text { assesstment }\end{array}$ & $\mathrm{N}: 25 ; 2$ male & $\begin{array}{l}\text { Observational } \\
\text { study }\end{array}$ & $\begin{array}{l}\text { FFI } 23 \\
\text { items } 3 \\
\text { domains }\end{array}$ & VAS scale & $\begin{array}{l}\text { FFI was to evaluate foot sensation } \\
\text { related to RA }\end{array}$ \\
\hline & & Rheumatoid arthritis foot & $\begin{array}{l}\text { Mean age: } 55 \\
(S D=9.9) R A ; \\
9.6(S D=7)\end{array}$ & & & & Conclusion: useful \\
\hline
\end{tabular}


Table 10 Studies using foot function measures in observational studies (Continued)

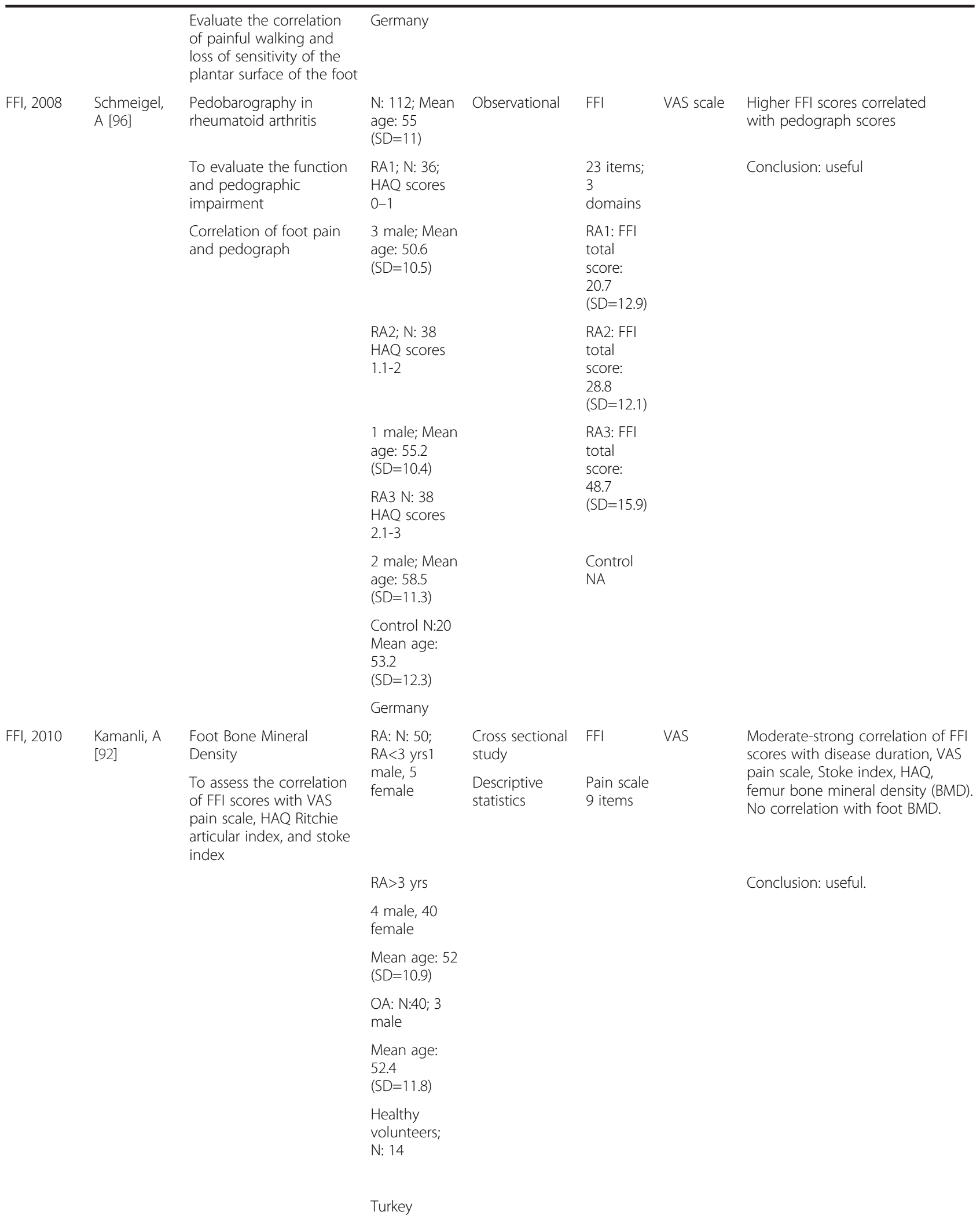


Table 10 Studies using foot function measures in observational studies (Continued)

\begin{tabular}{|c|c|c|c|c|c|c|c|}
\hline \multirow[t]{4}{*}{ FFI, 2010} & $\begin{array}{l}\text { Goldstein, } \\
\text { CL [94] }\end{array}$ & Foot and ankle trauma & N: 52; 31 male & $\begin{array}{l}\text { Cross sectional } \\
\text { study the } \\
\text { mean duration } \\
\text { post trauma } \\
15.5 \text { months } \\
\text { (1 month-10 } \\
\text { years) }\end{array}$ & $\mathrm{FFI}$ & VAS & $\begin{array}{l}\text { There was a high correlation } \\
\text { among FFI scores and the } 5 \text { listed } \\
\text { instruments. }\end{array}$ \\
\hline & & $\begin{array}{l}\text { Correlation of FFI, SF-12, } \\
\text { SMFA, FAAM, AAOS, } \\
\text { AOFAS }\end{array}$ & $\begin{array}{l}\text { Mean age: } \\
43.3(18-85)\end{array}$ & & 9 items & & Conclusion: useful \\
\hline & & & $\begin{array}{l}\text { OA; Foot and } \\
\text { ankle trauma }\end{array}$ & & Pain scale & & \\
\hline & & & Canada & & & & \\
\hline \multirow[t]{4}{*}{ FFI, 2010} & $\begin{array}{l}\text { Kavlak, Y } \\
\text { [93] }\end{array}$ & $\begin{array}{l}\text { Elderly men Concurrent } \\
\text { measure with VAS pain } \\
\text { scale, foot problem score, } \\
\text { hind foot function scale }\end{array}$ & N: 53; 53 male & $\begin{array}{l}\text { Cross sectional } \\
\text { study }\end{array}$ & $\mathrm{FFI}$ & VAS scale & $\begin{array}{l}\text { FFI was simple and comprehensive } \\
\text { and was significantly correlated } \\
\text { with hind foot function scale, and } \\
\text { scores of timed up and go. }\end{array}$ \\
\hline & & & $\begin{array}{l}\text { Mean age: } \\
73.8 \text { (7.08) }\end{array}$ & & 23 items & & Conclusion: useful \\
\hline & & & $\begin{array}{l}\text { Foot } \\
\text { problems }\end{array}$ & & $\begin{array}{l}3 \\
\text { domains }\end{array}$ & & \\
\hline & & & Turkey & & & & \\
\hline
\end{tabular}

scale was valid. Category B FFI as Criterion Validity. Articles in this category describe several health measures and use the FFI full scale or subscales to validate these measures. Bal et al. [26] found a strong correlation of FFI scores and scores of RA functional measures: the Health Assessment Questionnaire (HAQ) and Steinbrocker Functional Class (SFC). SooHoo et al. [27] found that the Rand 36-Item Short Form Health Survey (SF-36) scores of a sample of individuals with foot and ankle disorders were moderately correlated with FFI scores and concluded that FFI scores can be used to monitor the quality of life of these patients. Shrader et al. [28] measured the stability of navicular joint alignment and found that this measure correlated well with the FFI scores of the sample. Helliwell et al. [29] developed a new measure, the Foot Impact Scale (FIS), to measure the impact of foot problems on foot health in a sample of individuals with RA; the metric of FIS was validated with the FFI and HAQ. In an RA study, van der Leeden et al. [30] reported that Western Ontario and McMaster Universities Osteoarthritis Index (WOMAC) and Disease Activity Scores in 44 joints (DAS 44) were correlated with FFI scores; furthermore, this author discerns the correlations that the FFI pain subscale scores correlated with forefoot pain while the FFI function subscale scores correlated with hindfoot problems. The FFI scores were also used as validation measures of the American Orthopedic Foot and Ankle Society (AOFAS) clinical rating scales, an instrument that was widely used by foot and ankle surgeons [31]. These validation studies were reported by Baumhauer et al. [32] for the AOFAS hallux clinical rating scale and by Ibrahim et al. [33] for the AOFAS clinical rating scale, which was well to moderately correlated with FFI scores. The latter finding was based on his study with a $41 \%$ response rate in a sample consisting of 45 individuals.

Table 11 Reliability and unidimensionality of the full scale, short form, and subscales

\begin{tabular}{|c|c|c|c|c|c|c|c|}
\hline & $\begin{array}{l}\text { Full scale } \\
\text { (68 items) }\end{array}$ & $\begin{array}{l}\text { Short form } \\
\text { (34 items) }\end{array}$ & $\begin{array}{l}\text { 1-11 } \\
\text { (Pain) }\end{array}$ & $\begin{array}{l}12-19 \\
\text { (Stiffness) }\end{array}$ & $\begin{array}{l}20-39 \\
\text { (Difficulty) }\end{array}$ & $\begin{array}{l}40-49 \\
\text { (Limitation) }\end{array}$ & $\begin{array}{l}50-68 \\
\text { (Social issues) }\end{array}$ \\
\hline Person Reliability & .96 & .95 & .89 & .89 & .94 & .78 & .84 \\
\hline Cronbach's Alpha & .98 & .97 & .93 & .95 & .97 & .87 & .94 \\
\hline \multirow{3}{*}{$\begin{array}{l}\text { Unidimensionality Criteria } \\
\text { (Ratio of the raw variance explained by measures: } \\
\text { Unexplained variance in } 1^{\text {st }} \text { contrast } \geq 3 \text { ) }\end{array}$} & $56.8 / 10.6=$ & $60.2 / 15.8=$ & $66.7 / 22.1=$ & $67.5 / 34.7=$ & $72.7 / 15.5=$ & $63.4 / 19.2=$ & $53.6 / 18.1=$ \\
\hline & 5.4 & 3.8 & 3.0 & $1.94^{1}$ & 4.69 & $3.3^{2}$ & $2.96^{3}$ \\
\hline & Yes & Yes & Yes & No & Yes & Yes & No \\
\hline
\end{tabular}

${ }^{1}$ Further inspection of the data revealed that the two-factor solution was associated with the severity of the items, where the two factors were actually low and high severity stiffness, i.e. opposite poles of the same factor. Therefore, the scale is useful as a measure of stiffness.

2 These were the results after removing item 41 (ASSISTO).

${ }^{3}$ Approximately unidimensional. 
Table 12 Summary of FFI and FFI-R uses as provided in detail in Tables 5-10

\begin{tabular}{llll}
\hline $\begin{array}{l}\text { FFI/FFI-R } \\
\text { instrument } \\
\text { usage }\end{array}$ & Category & $\begin{array}{l}\text { Name of } \\
\text { instrument }\end{array}$ & $\begin{array}{l}\text { First Author's } \\
\text { name [reference } \\
\text { number] }\end{array}$ \\
\hline $\begin{array}{l}\text { Measurement } \\
\text { (Details in }\end{array}$ & A) New & FFI & Budiman- Mak E [7]
\end{tabular}

Tables 5 \& 6) Instrument

\begin{tabular}{|c|c|c|}
\hline & FFI-R & Budiman-Mak E [11] \\
\hline & FFI-site to site & Saag KG [23] \\
\hline & AOS & Domsic RT [24] \\
\hline & FFI Likert Scale & Agel J [25] \\
\hline \multirow[t]{8}{*}{$\begin{array}{l}\text { B) FFI as Criterion } \\
\text { Validity }\end{array}$} & HAQ and SFC & Bal A [26] \\
\hline & SF-36 & SooHoo NF [27] \\
\hline & $\begin{array}{l}\text { Navicular joint } \\
\text { alignment }\end{array}$ & Shrader JA [28] \\
\hline & FIS & Helliwell P [29] \\
\hline & $\begin{array}{l}\text { WOMAC and } \\
\text { DAS } 44\end{array}$ & $\begin{array}{l}\text { Van der Linden M } \\
\text { [30] }\end{array}$ \\
\hline & AOFAS & Lau JT [31] \\
\hline & AOFAS Hallux & Baumhauer JF [32] \\
\hline & AOFAS & Ibrahim T [33] \\
\hline \multirow[t]{4}{*}{$\begin{array}{l}\text { C) Cultural } \\
\text { adaptation/ } \\
\text { Translation }\end{array}$} & Dutch-FFI-5pts & $\begin{array}{l}\text { Kuyvenhoven MM } \\
\text { [3] }\end{array}$ \\
\hline & $\mathrm{FFI-G}$ & Naal FD [34] \\
\hline & $\begin{array}{l}\text { FFl-Taiwan } \\
\text { Chinese }\end{array}$ & Wu SH [36] \\
\hline & FFI- Spanish & MAPI Institute [38] \\
\hline
\end{tabular}

Surgeries

(Details in Table 7)

\begin{tabular}{|c|c|c|}
\hline $\begin{array}{l}\text { a) Arthrodeses } \\
\text { and Fusions }\end{array}$ & FFI, FFI-Dutch, & $\begin{array}{l}\text { Lin SS [39], } \\
\text { Grondal L [40],van } \\
\text { der Krans A [41], } \\
\text { Stegman M [42], } \\
\text { Vesely R [43], } \\
\text { Doets HC [44], } \\
\text { Jung HG [45], van } \\
\text { Doeselaar DJ [46], } \\
\text { Niki H [47] }\end{array}$ \\
\hline b) Arthroplasty & $\begin{array}{l}\mathrm{FFI}, \mathrm{FFI} \text { pain } \\
\text { and difficulty } \\
\text { subscales, }\end{array}$ & $\begin{array}{l}\text { Ibrahim T [48], } \\
\text { Taranow WS [49], } \\
\text { Schutte BG [50], } \\
\text { Bonnin MP [51] }\end{array}$ \\
\hline c) Fracture Care & $\mathrm{FFI}$ & $\begin{array}{l}\text { Vallier HA [52], } \\
\text { Harris AM [53], } \\
\text { Potter MQ [54], } \\
\text { Gaskill T [55] }\end{array}$ \\
\hline $\begin{array}{l}\text { d) Reconstruction } \\
\text { Surgery }\end{array}$ & FFI, FFI-Dutch & $\begin{array}{l}\text { Mulcahy D [56], } \\
\text { Ward CM [57], } \\
\text { Schlegel UJ [58], } \\
\text { van der Heide HJ } \\
\text { [59], Kroon M [60] }\end{array}$ \\
\hline e) Other surgery & $\begin{array}{l}\mathrm{FFI}, \mathrm{FFI} \text { disability } \\
\text { subscale, } \mathrm{FFI} \\
\text { pain subscale, } \\
\mathrm{FFI} \text { pain and }\end{array}$ & $\begin{array}{l}\text { Watson TS [61], } \\
\text { Daniels TR [62], Lee } \\
\text { S [63], SooHoo NF } \\
\text { [64], Stropek S [37], }\end{array}$ \\
\hline
\end{tabular}

Table 12 Summary of FFI and FFI-R uses as provided in detail in Tables 5-10 (Continued)

\begin{tabular}{|c|c|c|c|}
\hline & & $\begin{array}{l}\text { disability } \\
\text { subscales }\end{array}$ & $\begin{array}{l}\text { Castellani C [65], } \\
\text { Aurich M [66], Eberl } \\
\text { R [67]. }\end{array}$ \\
\hline \multicolumn{4}{|l|}{ Orthoses } \\
\hline \multirow[t]{3}{*}{$\begin{array}{l}\text { (Details in } \\
\text { Table 8) }\end{array}$} & a) Forefoot & $\mathrm{FFI}$ & $\begin{array}{l}\text { Caselli MA [68], de } \\
\text { P Magalahaes [69], } \\
\text { Conrad KJ [70], } \\
\text { William AE [71], } \\
\text { Cho NS [72], Welsh } \\
\text { BJ [73], Budiman- } \\
\text { Mak E [74]. }\end{array}$ \\
\hline & b) Mid foot & FFI-R & $\begin{array}{l}\text { Rao S [75], Rao S } \\
{[76]}\end{array}$ \\
\hline & c) Hind foot & $\begin{array}{l}\mathrm{FFI}, \mathrm{FFI}, \text { Brazil } \\
\text { (pain subscale } \\
\text { modified), }\end{array}$ & $\begin{array}{l}\text { Caselli MA [77], } \\
\text { Baldassin V [35], } \\
\text { Pfeffer G [78], } \\
\text { Gross MT [79], } \\
\text { Woodburn J [80], } \\
\text { Lin J [81], Slattery } \\
\text { M [82], Powell M } \\
\text { [83], Novak P [84], } \\
\text { Clark H [85] }\end{array}$ \\
\hline \multicolumn{4}{|c|}{$\begin{array}{l}\text { Other } \\
\text { interventions }\end{array}$} \\
\hline \multirow[t]{2}{*}{$\begin{array}{l}\text { (Details in } \\
\text { Table 9) }\end{array}$} & Injection & $\begin{array}{l}\text { FFI pain and } \\
\text { disability } \\
\text { subscales }\end{array}$ & Cui Q [86] \\
\hline & $\begin{array}{l}\text { Stretching } \\
\text { exercise }\end{array}$ & $\begin{array}{l}\mathrm{FFI}, \mathrm{FFI} \text { pain } \\
\text { subscale }\end{array}$ & $\begin{array}{l}\text { DiGiovani BF [87], } \\
\text { Kulig K [88], } \\
\text { Rompe JD [89]. }\end{array}$ \\
\hline
\end{tabular}

Observational studies

\begin{tabular}{llll}
$\begin{array}{l}\text { (Details in } \\
\text { Table 10) }\end{array}$ & Foot morbidities & & \\
& $\begin{array}{l}\text { In diabetes } \\
\text { mellitus }\end{array}$ & $\begin{array}{l}\text { FFl pain } \\
\text { subscale }\end{array}$ & Novak P [4] \\
& In rheumatic & FFI & Williams AE [90], \\
diseases & & Williams AE [91] \\
In bone mineral & FFl pain & Kamanli A [92] \\
density & subscale & \\
In elderly & FFI & Kavlak Y [93] \\
In foot post-injury & FFl pain & Goldstein CL [94] \\
& subscale & \\
In rheumatoid & FFl & Rosenbaum D [95], \\
arthritis & & Schmeigel A [96] \\
\hline
\end{tabular}

Category C Cultural Adaptation or Translation. The first translation of the FFI was the Dutch-language instrument known as Dutch FFI-5pts [3]. The German-language translation of the instrument is the FFI-G [34]; the FFI was also translated into Brazilian Portuguese [35], Taiwan Chinese [36], Turkish [26], and Czech [37]. There was also a Spanish translation conducted by the MAPI Institute in Lyon, France [38]. These translations complied with rigorous language translation procedures; occasionally, some item adjustments of the scales were needed. In summary, the 


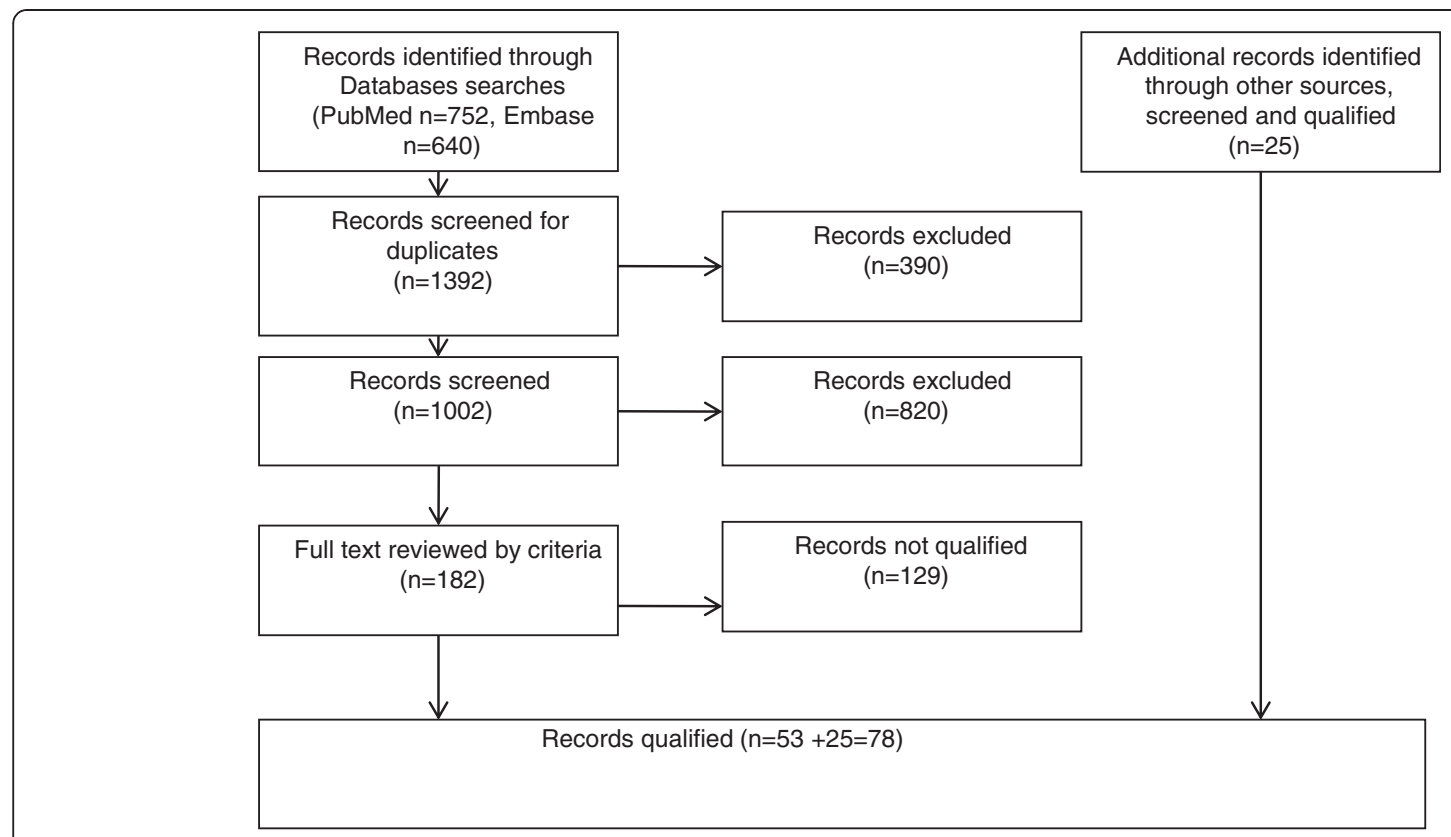

Figure 1 Algorithm of searched and screened for qualified paper.

FFI was developed with good reliability and validity; it also inspired and served as criterion validity for newer foot health measures and attracted the attention of researchers around the world, who conducted translations and adaptations of the tool into their native languages and cultures.

Table 6 is a supplement to Table 5 and displays the clinimetrics of the instruments listed in Table 5; measures were metrically good, with reliability and validity values greater than 0.7 with one exception where the pain subscale had a reliability of 0.64 [3].

\section{Surgical intervention}

The FFI is one of the outcome measures most frequently used by AOFAS members [31]. It was first used to measure surgical outcomes. The surgical interventions and outcomes are summarized in Table 7. There are 30 articles, categorized generally according to type and location of surgical procedure. Five distinct procedural categories were identified as follows: (a) arthrodeses within the foot or ankle [39-47], (b) arthroplasty within the foot or ankle [48-51], (c) fracture care of the foot or ankle [52-55], (d) deformity reconstruction surgery of the foot or ankle [56-60], and (e) various surgical interventions for chronic conditions [61-64]. The FFI was also used to assess outcomes of less invasive procedures, such as calcaneal spur treatment by arthroscopy [37], distal tibia repair using fixation with cannulation osteosyntheses [65], arthroscopic chondrocyte implant of the tibia and fibula [66], and surgical interventions for complex ankle injuries [67]. In summary, the FFI and the Dutch FFI5 pts appeared to be useful in measuring outcomes of various surgical procedures in children, adults, and individuals with acute, chronic, and congenital foot and ankle problems.

\section{Orthotic interventions}

Table 8 lists studies using foot function outcome measures in orthotic interventions in the foot and ankle. The studies assessed the impact of orthotic treatment on forefoot, midfoot, and hindfoot/ankle pathology. Orthotic treatment on the forefoot in patients with RA improved the scores for pain, disability and activities $[68,69]$, however the scores were unchanged in the study by Conrad et al. [70]. Other studies using special shoes and shoe inserts showed symptoms of relief in hallux valgus pain [71] hindfoot and forefoot problems [72,73]; and slowing the progression of hallux valgus in early RA [74]. Midfoot studies assessing the treatment of full length orthoses on pain relief [75], and mobility were performed using the FFI-R as an outcome measures [76]. For hindfoot conditions treatment with orthoses included studies of heel pain [77], plantar fasciitis [35,78,79], stabilizing hindfoot valgus [80], correction of posterior tibialis tendon dysfunction [81], destructive hemophilic arthropathy of the foot and ankle [82] and juvenile idiopathic arthritis of the foot and ankle [83]. Shoes/shoe inserts have also been found to relieve foot and ankle pain from arthritides $[84,85]$. In summary, the FFI and FFI-R clearly provided useful outcome measures for orthotic management of a wide range of foot and ankle disorders. 


\section{Medical intervention}

The FFI also was used to measure foot health outcomes associated with medical interventions (Table 9), such as cortisone injection of the ankle adhesive capsulitis [86]; the injection resulted in improved FFI pain and disability subscale scores. Di Giovanni et al. [87] measured the outcome of stretching exercises for plantar fasciitis versus Achilles tendonitis; both groups showed improvement in FFI pain subscale scores. Kulig et al. [88] used the FFI pain and disability subscales to measure the outcomes of exercise intervention in posterior tibial tendon dysfunction. Rompe et al. [89] reported the FFI pain score improved in the stretching treatment group of a randomized clinical trial using stretching and shockwave therapy to treat patients with plantar fasciopathy. Overall, the FFI was useful in measuring the outcomes of conservative interventions in chronic foot and ankle conditions.

\section{Observational studies}

Investigators had chosen the FFI scores or the subscale scores to determine the prevalence and disease burden of foot and ankle conditions in the general population (Table 10). Novak et al.[4] used FFI scores to evaluate type 2 diabetes with and without neuropathy and identified that group with neuropathy had worse FFI scores. Williams and Bowden [90] correlated high FFI scores to foot morbidity in rheumatic diseases, and estimated cost of care/staffing concerns for that patient subset. Williams [91] also used the FFI scores in patients with Paget's disease and noted the impacts on plantar foot pressures, gaits, and ambulation abilities. Kamanli et al. [92] correlated the scores of the FFI and foot bone mineral density, then extrapolated these scores to that individual's skeletal bone density. Kavlak and Demitras [93] reported a strong correlation of FFI scores with the scores of VAS pain scale, foot pain scale (FPS), and hindfoot function scale (HFS) in patients with foot problems. Goldstein et al. [94] noted that FFI scores of individuals with previous foot injuries had a high correlation with 6 other foot function instruments. Rosenbaum et al. [95] found that plantar sensory impairment of the foot in patients with RA was correlated with poor FFI scores. Schmiegel et al. [96] found that pedobarograph scores of patients with RA with foot pain were correlated with poor FFI and HAQ scores. In summary, FFI scores were useful in detecting the prevalence of foot and ankle problems and as a measure of concurrent validity for other foot health measures in various chronic foot conditions.

In all, we found the FFI instrument was frequently chosen as an outcome measure of surgical, orthotic, and medical treatments, but its application was wider than we originally imagined. It was not limited to outcome measures; FFI scores were also applied in the promotion of foot health as a common public health issue and in increasing the awareness of health system administrators. The FFI was also used in the validation of newly developed foot health measures.

\section{Objective 3: The strengths and weaknesses of the FFI and FFI-R as reported in the literature}

FFI: The FFI questionnaire had good psychometric properties [97-100], and the pain subscale was sensitive to change during instrument development [13]. In a study about treatment of plantar fasciitis in individuals with chronic foot pain, SooHoo et al. [64] reported that the pain subscale of the FFI had high standard response mean (SRM) and high effect size (ES) as outcome measures of surgery in chronic foot and ankle problems. While Landorf and Radford measured the clinical ability to detect a change as minimal important difference (MID) in plantar fasciitis [101]. All these clinical measures add to the credibility of the FFI as a self-reporting measure, the FFI reflects patients' assessment of their symptoms/health status, which directs providers about proper care planning and progress toward treatment goals. FFI is one of the most cited measures of its kind [102].

There are weaknesses of the FFI. During the development of the index, clinicians generated the questionnaire items without patient participation [13,97]; therefore, items might not fully reflect patients' needs, might be sex biased [7], and might not be applicable to high-functioning individuals. A theoretical model was not part of the design, nor were the items related to footwear $[13,103]$, which are essential to support the construct of this instrument. It is also lacking items for measuring quality of health and satisfaction with care; however, these items can be appended as a global statement in the questionnaire. In all, the FFI has been the most studied and widely used foot-specific self-reporting measure; however, further testing by gender, age, race, language, etc. would provide assurance of its generalizability.

FFI-R: The FFI-R was developed in response to criticism of the FFI and to address issues of contemporary interest. Most original items from the FFI were selected in the development of FFI-R, and new items about footwear and psychosocial factors were added, which improved its construct coverage. Patients and clinicians were involved in the generation of items. Its design closely followed the ICF theoretical model [13]; its psychometric properties are strong and are based on the IRT 1-parameter or the Rasch measurement model. It was designed to be a comprehensive measure of foot health-related quality of life, with both long and short forms [99], allowing clinicians and researchers to choose the measures they need for the intended study. Although the FFI-R did not include information on clinical ability to measure change in its development, Rao et al. [75,76] did measure the minimal detectible change (MDC) and 
the effect size, in individuals with midfoot arthritis, which also added to the credibility of its metrics.

\section{Objective 4: The newly analyzed FFI-R with improved psychometric values \\ The full scale and short form}

For the FFI-R L (68 items) [11], person reliability was high: 0.96 , respectively. In the PCA, $56.8 \%$ of the variance was explained by the measure, with only $10.6 \%$ of the variance explained by the first factor of residuals. These findings support that the full FFI-R meets the unidimensionality requirement of the Rasch model. Further, the criterion for unidimensionality was a ratio of the raw variance in the first contrast of residuals that was 5.4 (i.e., greater than 3). For the FFI-R S (34 items) [11], person reliability was 0.95 , similar to the reliability estimates of the FFI-R L. The PCA of the FFI-R $S$ revealed that unidimensionality criteria were also satisfied. This supports the use of a short form of the measure, because the item response burden on patients is lower, at 34 questions. Because this measure is as reliable as the full measure, its use is supported for clinical settings.

\section{Subscales}

All subscales of the FFI-R had strong person reliability estimates (Table 11), ranging from 0.78 to 0.94 for person reliability. The PCA indicated that unidimensionality held for each subscale, with the exception of the stiffness subscale. Further inspection of the data revealed that the twofactor solution reflected groups of the low-severity and high-severity items and was not the result of a competing factor. Unidimensionality for the limitation subscale was met after dropping item 41 (ASSISTO), an item listed in the FFI-R database. Overall, the subscales of the FFI-R satisfied unidimensionality criteria and were reliable measures of the latent traits (Table 11).

\section{Response category analysis}

The response category analyses for each of the subscales (done after collapsing Categories 5 and 6) revealed that, for the first three subscales (pain, stiffness, and difficulty), the response categories behaved as required by the Rasch model. However, for the subscales of limitation and social issues (both of which are time scales), there was some indication that respondents had difficulty distinguishing between, " 2 = A little of the time," and, " 3 = Some of the time." We considered, then, collapsing these categories and making all FFI-R subscales have four possible response categories. This would ensure uniformity of the measure and decrease the burden on patient response. Therefore, the first three subscales, which measure severity, " 3 = Severe pain," " $4=$ Very severe pain," and " $5=$ Worst pain imaginable," were collapsed. This was justified because all three captured the notion of severe pain. Overall, the analyses showed that the response to each item functioned well with the fouritem response categories.

\section{Discussion}

This review evaluated 78 eligible articles (Figure 1). In the past 20 years, it appears that the FFI and FFI-R were widely used across national and international clinical and research communities. The instruments were administered to over 4700 study participants of males and females worldwide, across age groups, with 20 different diagnoses consisting of congenital, inflammatory/degenerative, acute and chronic foot and ankle problems. The FFI was also incorporated into other newer foot health measures $[23,24]$, and also underwent changes in the measurement scale from VAS to Likert scale such as the one conducted by Agel et al. [25]. The scale changes also occurred in FFI adaptation to the Dutch [3], German [34], and Taiwanese Chinese [36] including our revised FFI-R [11] to give a few examples. The strong metrics of FFI subscales and full scale (Table 12, Category A), facilitated the investigator's choice to use its subscale(s) or full scale in clinical or research applications as appropriate. The FFI was also frequently used as validation criterion for other foot health measures (Table 12, Category B); this validation usage has elevated the credibility of the FFI as an outcome measure for foot and ankle problems. Since the FFI was developed using CTT procedures, it is sample and content dependent, therefore its metrics were tested in many different samples, where its metrics were proven to be consistently strong. The exception was in the study of Baumhauer et al. [32] where high foot functioning was evident in the sample; therefore, investigators should exercise caution in the interpretation of this result. While the FFI was developed initially as disease specific for early RA, in later years, it was used in many non-RA foot and ankle problems and was proven to be a valid measure as well. The FFI and FFI-R were frequently used as outcome measures in surgical and clinical interventions with positive results (Tables 7, 8, 9, and 10). The FFI scores were also used in many observational studies (Table 10) and those reports might be helpful for researchers and the health system administrators in establishing a health policy. Although the FFI was extensively studied and generally received positive ratings $[23,29,102]$, we realized the need for improvement in the measures of FFI and FFI-R and have discussed this issues comprehensively under Objective 3 in this paper. We conducted a re-analysis and made improvements to the metrics and scales of FFI-R as presented in Table 11 and questionnaires FFI-R Long Form (See Additional file 1), and Short Form (See Additional file 2).

In recent articles about FFI used as outcome measures, the authors have included the clinical measures; the effect size, and standard response mean [64], and minimal important difference [101], while Rao et al. reports minimal 
detectible change and effect size of the FFI-R [75], all these have increased the credibility of the clinical use of the FFI to help in power analysis and sample size estimation for future studies.

\section{Limitations of this review}

Our literature search was limited to publications written in the English language and covered only publications until 2010; therefore, this might exclude the FFI- and FFI-R-related published articles not written in English, as well as those more recent articles published in English.

\section{Conclusions}

The FFI pioneered measuring outcomes in foot health. This instrument has been tested through time and adapted in its measures as it was frequently used in full scales or subscales to measure outcomes in various clinical practice or research studies. The FFI has also had a role in shifting the paradigm from a reliance on physical and biochemical findings as outcomes to the use of outcomes that are relevant to patients. Thus, the measure established patient-centered, valid, reliable, and responsive hard data endpoints. The rating scales also underwent changes; for practicality and userfriendliness in clinical and research settings. The FFI was recognized as a valid instrument and used as a validation criterion of other measures. It was adapted and translated into multiple languages. It was applied to all age groups, across genders and was useful in measuring varied medical and surgical conditions.

In realizing the scope of FFI applications, we acknowledge the contributions of friends and colleagues around the world who not only used the FFI in their studies but also made adaptations and translations to make the FFI a versatile instrument in promoting and maintaining foot health. The FFI-R has good psychometric properties and is available in long and short forms for ease of clinical use. In response to findings in this review, we conducted a rigorous analysis to strengthen the metrics of the FFI-R and changed the rating scales to be more user-friendly and practical.

Both the FFI and FFI-R are in the public domain and permission to use them is free of charge. They are available from the developers of these instruments and from the AOFAS web site. These instruments are self-administered and are written at an eighth-grade reading level. The FFI scores are interpreted as $0 \%-100 \%$ for each subscale and the overall score. Higher FFI and FFI-R scores indicate poor foot health and poor foot health-related quality of life. The FFI and FFI-R put minimal burden on respondents and the questionnaires are not emotionally sensitive. The administrative burden is also minimal and it does not require formal training to score or to interpret [104]. Translations and adaptations are available in Dutch [3], Taiwan Chinese [36], German [34], Turkish [26], Brazilian Portuguese [35], and Spanish [38].
This review attests to the widespread use of foot health measures, and we have noticed the advancement of foot health in general across diagnoses. It has been a privilege for us to serve patients, clinicians, and researchers to fulfill the mission in improving foot health through the use of the FFI and FFI-R. These instruments are available for users, and can be downloaded as they are presented as electronic files.

\section{Additional files}

Additional file 1: Revised FOOT FUNCTION INDEX (FFI-R) Additional file 2: Revised FOOT FUNCTION INDEX (FFI-R) Short Form.

\section{Abbreviations}

AOFAS: American Orthopedic Foot and Ankle Society; CTT: Classical test theory; EMBASE: Excerpta Medica Database; FFI: Foot Function Index; FFIR: Foot Function Index Revised; EBM: Elly Budiman-Mak; FFI-R L: Foot Function Index Revised Long Form; FFI-R S: Foot Function Index Revised Short Form; HAQ: Health Assessment Questionnaire; IRT: item response theory; JM: Jessica Massa; KJC: Kendon J Conrad; Medline: Medical Literature Analysis and Retrieval System; PUBMED: public Medline; RA: rheumatoid arthritis; RMS: Rodney M. Stuck; VAS: visual analog rating scale; AAOS: American Academy of Orthopedic Surgeon; ANOVA: Analysis of Variance; AOS: Ankle Osteoarthritis Index; BMD: Bone Mineral Density; CA: Crohnbach's Alpha; CRI: Clinical Rating Index; CV: Calcaneal Varus; DAS 44: Disease Activity Score in 44 joints of patient with rheumatoid arthritis (RA); DX: Diagnosis; EF: External Fixation Procedure; ES: Effect Size; FAAM: Foot and Ankle Ability Measure; FFI-5pts: Dutch Foot Function Index with 5 point Likert Scale; FFI-G: Foot Function Index - German Language; FHSQ: Foot Health Status Questionnaire; FIS: Foot Impact Scale; FPS: Foot Problem Score; FSI: Foot Structure Index; FX: Fracture; HFS: Hind Foot Function Scale; HMIP: Hallux Metatarso-interphalangeal Joint; HR: Hallux Rigidus; ICC: Interclass Correlation Coefficient; JIA: Juvenile Idiopathic Arthritis; JRA: Juvenile Rheumatoid Arthritis; LMIP: Lesser Metatarso-interphalangeal Joint; MCS: Mental Component Score of SF-36; MDC: Minimal Detectible Change; MFA: Musculoskeletal Function Assessment; MFDQ: Manchester Foot Disability Questionnaires; MID: Minimal Important Difference;

MODEMS: Musculo-skeletal Outcome Data Evaluation and Management System; MTP: Metatarsophalangeal Joint; NA: Not Applicable;

OA: Osteoarthritis; PAS: Physical Activity Scale; PCS: Physical Component Score of SF-36; PedQL: Pediatric Quality of Life Scale; PF: Plantar Fasciitis; PTTD: Posterior Tibialis Tendon Dysfunction; QOL -12: Quality of Life 12 items short form; RAl: Ritchie Articular Index; RCT: Randomized Control Trial; SD: Standard Deviation; SF-36: Rand 36 items health survey form; SF-36 MCS: Mental Component Score of SF-36; SF-36 PCS: Physical Component Score of SF-36; SF-12: Rand 12 items short form health survey;

SFC: Steinbrocker Functional Class; SMFA: Musculoskeletal Function Assessment; SRM: Standard Response Mean; SI: Stroke Index; TAA: Total Ankle Arthroplasty; TMT: Tarso Meta-metatarso Joint; UCLA: University of California Los Angeles; WOMAC: Western Ontario MacMaster University Osteo Arthritis Index.

\section{Competing interests}

The authors declare that they have no competing interests.

\section{Authors' contributions}

EBM, KJC, have contributed in drawing the concept and design of this paper, EBM initiated the literature search, reviewed, scrutinized them, and collected the abstracts and organized into tables. KJC, RMS and JM reviewed the tables and all authors participated in drafting the manuscript. KJC and JM also reanalyzed the original FFI-R data and revised the subscales and FFI-R response categories. All authors participated in revising the manuscript and have given final approval of the version to be published. 


\section{Acknowledgements}

The authors gratefully acknowledge the support from the Center for Management of Complex Chronic Care, Hines VA Hospital, Hines, IL, USA. The paper presents the findings and conclusions of the authors; it does not necessarily represent the Department of Veterans Affairs or Health Services Research and Development Service. We are also grateful to Cindi Fiandaca and the Hines VA medical library staff for assisting in the literature search, Madeline Thornton for assisting in designing the tables, Leahanne Sarlo and Mary Reidy for editing the manuscript.

\section{Author details}

${ }^{1}$ Center for Management of Complex Chronic Care, Staff Physician, Medical Service, Hines, VA Hospital, 5000 South 5th Ave, Hines, IL 60141-3030, USA. ${ }^{2}$ Department of Medicine Loyola University Stritch School of Medicine, Loyola University of Chicago, Maywood, IL 60513, USA. ${ }^{3}$ Health Policy and Administration (MC 923) School of Public Health University of Illinois at Chicago, 1603 West Taylor Street, Chicago, IL 60612-4394, USA. ${ }^{4}$ University of Illinois at Chicago School of Public Health (MC923), 1603 West Taylor Street, Chicago, Illinois 60612, USA. ${ }^{5}$ Department of Orthopaedic Surgery, Loyola University Stritch School of Medicine, Loyola University of Chicago, 2160 South First Ave, Maywood, IL 60153, USA. ${ }^{6}$ Surgical Service, Hines VA Hospital, 5000 South 5th Ave, Hines, IL 60141-3030, USA.

Received: 2 November 2012 Accepted: 11 January 2013 Published: 1 February 2013

\section{References}

1. Benvenuti F, Ferrucci L, Guralnik JM, Gangemi S, Baroni A: Foot pain and disability in older persons: an epidemiologic survey. J Am Geriatr Soc 1995, 43:479-484.

2. Leveille SG, Guralnik JM, Ferrucci L, Hirsch R, Simonsick E, Hochberg MC: Foot pain and disability in older women. Am J Epidemiol 1998, 148:657-665.

3. Kuyvenhoven MM, Gorter KJ, Zuithoff P, Budiman-Mak E, Conrad KJ, Post MW: The foot function index with verbal rating scales (FFI-5pt): a clinimetric evaluation and comparison with the original FFI. J Rheumatol 2002, 29:1023-1028.

4. Novak P, Burger H, Marincek C, Meh D: Influence of foot pain on walking ability of diabetic patients. J Rehabil Med 2004, 36:249-252.

5. Menz HB, Lord SR: The contribution of foot problems to mobility impairment and falls in community-dwelling older people. J Am Geriatr Soc 2001, 49:1651-1656.

6. Menz HB, Lord SR: Foot pain impairs balance and functional ability in community-dwelling older people. J Am Podiatr Med Assoc 2001, 91:222-229.

7. Budiman-Mak E, Conrad KJ, Roach KE: The Foot Function Index: a measure of foot pain and disability. J Clin Epidemiol 1991, 44:561-570.

8. Nunally J, Bernstein I: Psychometric Theory. New York: McGraw-Hill; 1994.

9. Bennett PJ, Patterson C, Wearing S, Baglioni T: Development and validation of a questionnaire designed to measure foot-health status. J Am Podiatr Med Assoc 1998, 88:419-428.

10. Landorf KB, Keenan AM: An evaluation of two foot-specific, health-related quality-of-life measuring instruments. Foot Ankle Int 2002, 23:538-546.

11. Budiman-Mak E, Conrad K, Stuck R, Matters M: Theoretical model and Rasch analysis to develop a revised Foot Function Index. Foot Ankle Int 2006, 27:519-527.

12. International Classification of Impairments, Disabilities and Handicaps; [www.who.int/entity/classification/icf/en]

13. Walmsley S, Williams AE, Ravey M, Graham A: The rheumatoid foot: a systematic literature review of patient-reported outcome measures. J Foot Ankle Res 2010, 3:12.

14. Linacre JM: Winsteps Rasch Measurement (Version 3.72.0); 2011.

15. Linacre JM: Structure in Rasch residuals:Why principal components analysis (PCA)? Rasch Measurement Transactions 1998, 1:636.

16. Linacre JM: Detecting multidimensionality: which residuals data-type works best? J Outcome Meas 1998, 2:266-283.

17. Smith EW Jr: Detecting and evaluating the impact of multidimensionality using item fit statistics and principal component analysis of residuals. J Appl Meas 2002, 3:205-231.

18. Reckase M: Unifactor latent trait model applied to multifactor tests: results and implications. J Educ and Behav Stat 1979, 4:207-230.

19. Embretson SE, Reise SP: Item response theory for psychologists. Mahwah, NJ: Lawrence Erlbaum Associates Inc; 2000.
20. Bond TG, Fox CM: Applying the Rasch model: fundamental measurement in the human sciences. Mahwah, NJ: Lawrence Erlbaum Associates; 2007.

21. Linacre JM: Investigating rating scale category utility. J Outcome Meas 1999, 3:103-122.

22. Linacre JM: Optimizing rating scale category effectiveness. J Appl Meas 2002, 3:85-106.

23. Saag KG, Saltzman CL, Brown CK, Budiman-Mak E: The Foot Function Index for measuring rheumatoid arthritis pain: evaluating side-to-side reliability. Foot Ankle Int 1996, 17:506-510.

24. Domsic RT, Saltzman CL: Ankle osteoarthritis scale. Foot Ankle Int 1998, 19:466-471.

25. Agel J, Beskin JL, Brage M, Guyton GP, Kadel NJ, Saltzman CL, Sands AK, Sangeorzan BJ, Soohoo NF, Stroud CC, et al: Reliability of the Foot Function Index: a report of the AOFAS Outcomes Committee. Foot Ankle Int 2005, 26:962-967.

26. Bal A, Aydog E, Aydog ST, Cakci A: Foot deformities in rheumatoid arthritis and relevance of foot function index. Clin Rheumatol 2006, 25:671-675

27. Soohoo NF, Samimi DB, Vyas RM, Botzler T: Evaluation of the validity of the Foot Function Index in measuring outcomes in patients with foot and ankle disorders. Foot Ankle Int 2006, 27:38-42.

28. Shrader JA, Popovich JM Jr, Gracey GC, Danoff JV: Navicular drop measurement in people with rheumatoid arthritis: interrater and intrarater reliability. Phys Ther 2005, 85:656-664.

29. Helliwell P, Reay N, Gilworth G, Redmond A, Slade A, Tennant A, Woodburn J: Development of a foot impact scale for rheumatoid arthritis. Arthritis Rheum 2005, 53:418-422.

30. VanderLeeden M, Steultjens M, Dekker JH, Prins AP, Dekker J: Forefoot joint damage, pain and disability in rheumatoid arthritis patients with foot complaints: the role of plantar pressure and gait characteristics. Rheumatology (Oxford) 2006, 45:465-469.

31. Lau JT, Mahomed NM, Schon LC: Results of an Internet survey determining the most frequently used ankle scores by AOFAS members. Foot Ankle Int 2005, 26:479-482.

32. Baumhauer JF, Nawoczenski DA, DiGiovanni BF, Wilding GE: Reliability and validity of the American Orthopaedic Foot and Ankle Society Clinical Rating Scale: a pilot study for the hallux and lesser toes. Foot Ankle Int 2006, 27:1014-1019.

33. Ibrahim T, Beiri A, Azzabi M, Best AJ, Taylor GJ, Menon DK: Reliability and validity of the subjective component of the American Orthopaedic Foot and Ankle Society clinical rating scales. J Foot Ankle Surg 2007, 46:65-74.

34. Naal FD, Impellizzeri FM, Huber M, Rippstein PF: Cross -culteral adaptation and validation of the Foot function Index for use in German -speaking patients with foot complaints. Foot Ankle Int 2008, 12:1222-1228.

35. Baldassin V, Gomes CR, Beraldo PS: Effectiveness of prefabricated and customized foot orthoses made from low-cost foam for noncomplicated plantar fasciitis: a randomized controlled trial. Arch Phys Med Rehabil 2009, 90:701-706.

36. Wu SH, Liang HW, Hou WH: Reliability and validity of the Taiwan Chinese version of the Foot Function Index. J Formos Med Assoc 2008, 107:111-118.

37. Stropek S, Dvorak M: Arthroscopic treatment for calcaneal spur syndrome. Acta Chir Orthop Traumatol Cech 2008, 75:363-368.

38. Foot Function Index Spanish Translation. [www.progolid.org]

39. Lin SS, Bono CM, Treuting R, Shereff MJ: Limited intertarsal arthrodesis using bone grafting and pin fixation. Foot Ankle Int 2000, 21:742-748.

40. Grondal L, Hedstrom M, Stark A: Arthrodesis compared to Mayo resection of the first metatarsophalangeal joint in total rheumatoid forefoot reconstruction. Foot Ankle Int 2005, 26:135-139.

41. van der Krans A, Louwerens JW, Anderson P: Adult acquired flexible flatfoot, treated by calcaneocuboid distraction arthrodesis, posterior tibial tendon augmentation, and percutaneous Achilles tendon lengthening: a prospective outcome study of 20 patients. Acta Orthop 2006, 77:156-163.

42. Stegman M, Anderson PG, Lowerens JWK: Triple arthrodesis of the hindfoot, a short term prospective outcome study. Foot Ankle Surg 2006, $: 71-77$.

43. Vesely R, Prochazka V, Visna P, Valentova J, Savolt J: Tibiotalocalcaneal arthrodesis using a retrograde nail locked in the sagittal plane. Acta Chir Orthop Traumatol Cech 2008, 75:129-133.

44. Doets HC, Zurcher AW: Salvage arthrodesis for failed total ankle arthroplasty. Acta Orthop 2010, 81:142-147. 
45. Jung HG, Myerson MS, Schon LC: Spectrum of operative treatments and clinical outcomes for atraumatic osteoarthritis of the tarsometatarsal joints. Foot Ankle Int 2007, 28:482-489.

46. van Doeselaar DJ, Heesterbeek PJC, Louwerens JWK, Swierstra BA: Foot Function After Fusion of the First Metatarsophalangeal Joint. Foot Ankle Int 2010, 31:670-675.

47. Niki H, Hirano T, Okada H, Beppu M: Combination joint-preserving surgery for forefoot deformity in patients with rheumatoid arthritis. J Bone Joint Surg Br 2010, 92:380-386.

48. Ibrahim T, Taylor G: The new press-fit ceramic Moje metatarsophalangeal joint replacement; short-term outcomes. The Foot 2004, 14:124-128.

49. Taranow WS, Moutsatson MJ, Cooper JM: Contemporary Approaches to stage II and III Hallux Rigidus: The role of Metalic Hemiarthroplasty of the Proximal Phalanx. Foot Ankle Clinic N Am 2005, 10:713-728.

50. Schutte BG, Louwerens JW: Short-term results of our first 49 Scandanavian total ankle replacements (STAR). Foot Ankle Int 2008, 29:124-127.

51. Bonnin MP, Laurent JR, Casillas M: Ankle function and sports activity after total ankle arthroplasty. Foot Ankle Int 2009, 30:933-944.

52. Vallier HA, Nork SE, Barei DP, Benirschke SK, Sangeorzan BJ: Talar neck fractures: results and outcomes. J Bone Joint Surg Am 2004, 86-A:1616-1624.

53. Harris AM, Patterson BM, Sontich JK, Vallier HA: Results and outcomes after operative treatment of high-energy tibial plafond fractures. Foot Ankle Int 2006, 27:256-265.

54. Potter MQ, Nunley JA: Long-term functional outcomes after operative treatment for intra-articular fractures of the calcaneus. J Bone Joint Surg Am 2009, 91:1854-1860.

55. Gaskill T, Schweitzer K, Nunley J: Comparison of surgical outcomes of intra-articular calcaneal fractures by age. J Bone Joint Surg Am 2010, 92:2884-2889

56. Mulcahy D, Daniels TR, Lau JT, Boyle E, Bogoch E: Rheumatoid forefoot deformity: a comparison study of 2 functional methods of reconstruction. J Rheumatol 2003, 30:1440-1450.

57. Ward CM, Dolan LA, Bennett DL, Morcuende JA, Cooper RR: Long-term results of reconstruction for treatment of a flexible cavovarus foot in Charcot-Marie-Tooth disease. J Bone Joint Surg Am 2008, 90:2631-2642.

58. Schlegel UJ, Batal A, Pritsch M, Sobottke R, Roellinghoff M, Eysel P, Michael JW: Functional midterm outcome in 131 consecutive cases of surgical clubfoot treatment. Arch Orthop Trauma Surg 2010, 130:1077-1081.

59. van der Heide HJ, Louwerens JW: Reconstructing the rheumatoid forefoot. Foot Ankle Surg 2010, 16:117-121.

60. Kroon M, Faber FW, van derLinden M: Joint preservation surgery for correction of flexible pes cavovarus in adults. Foot Ankle Int 2010, 31:24-29.

61. Watson TS, Anderson RB, Davis WH, Kiebzak GM: Distal tarsal tunnel release with partial plantar fasciotomy for chronic heel pain: an outcome analysis. Foot Ankle Int 2002, 23:530-537.

62. Daniels TR, Thomas R, Bell TH, Neligan PC: Functional outcome of the foot and ankle after free fibular graft. Foot Ankle Int 2005, 26:597-601.

63. Lee S, James WC, Cohen BE, Davis WH, Anderson RB: Evaluation of hallux alignment and functional outcome after isolated tibial sesamoidectomy. Foot Ankle Int 2005, 26:803-809.

64. Soohoo NF, Vyas R, Samimi D: Responsiveness of the foot function index AOFAS clinical rating systems, and SF-36 after foot and ankle surgery. Foot Ankle Int 2006, 27:930-934

65. Castellani C, Riedl G, Eberl R, Grechenig S, Weinberg AM: Transitional fractures of the distal tibia: a minimal access approach for osteosynthesis. J Trauma 2009, 67:1371-1375.

66. Aurich M, Bedi HS, Smith PJ, Rolauffs B, Muckley T, Clayton J, Blackney M: Arthroscopic treatment of osteochondral lesions of the ankle with matrix-associated chondrocyte implantation: early clinical and magnetic resonance imaging results. Am J Sports Med 2011, 39:311-319.

67. Eberl R, Singer G, Schalamon J, Hausbrandt P, Hoellwarth ME: Fractures of the talus-differences between children and adolescents. J Trauma 2010, 68:126-130.

68. Caselli MA, Levitz SJ, Clark N, Lazarus S, Velez Z, Venegas L: Comparison of Viscoped and PORON for painful submetatarsal hyperkeratotic lesions. J Am Podiatr Med Assoc 1997, 87:6-10.

69. de PMagalhaes E, Davitt M, Filho DJ, Battistella LR, Bertolo MB: The effect of foot orthoses in rheumatoid arthritis. Rheumatology (Oxford) 2006, 45:449-453.

70. Conrad KJ, Budiman-Mak E, Roach KE, Hedeker D, Caraballada R, Burks D, Moore $\mathrm{H}$ : Impacts of foot orthoses on pain and disability in rheumatoid arthritics. J Clin Epidemiol 1996, 49:1-7.
71. Williams $A E$, Rome $K$, Nester $C$ : A clinical trial of specialist footwear for patients with rheumatoid arthritis. Rheumatology (Oxford) 2007, 46:302-307.

72. Cho NS, Hwang JH, Chang HJ, Koh EM, Park HS: Randomized controlled trial for clinical effects of varying types of insoles combined with specialized shoes in patients with rheumatoid arthritis of the foot. Clin Rehabil 2009, 23:512-521.

73. Welsh BJ, Redmond AC, Chockalingam N, Keenan AM: A case-series study to explore the efficacy of foot orthoses in treating first metatarsophalangeal joint pain. J Foot Ankle Res 2010, 3:17.

74. Budiman-Mak E, Conrad KJ, Roach KE, Moore JW, Lertratanakul Y, Koch AE Skosey JL, Froelich C, Joyce-Clark N: Can Foot Orthoses Prevent Hallux Valgus Deformity in Rheumatoid Arthritis? A Randomized Clinical Trial. J Clin Rheumatol 1995, 1:313-322.

75. Rao S, Baumhauer JF, Becica L, Nawoczenski DA: Shoe inserts alter plantar loading and function in patients with midfoot arthritis. J Orthop Sports Phys Ther 2009, 39:522-531.

76. Rao S, Baumhauer JF, Tome J, Nawoczenski DA: Orthoses alter in vivo segmental foot kinematics during walking in patients with midfoot arthritis. Arch Phys Med Rehabil 2010, 91:608-614.

77. Caselli MA, Clark N, Lazarus S, Velez Z, Venegas L: Evaluation of magnetic foil and PPT Insoles in the treatment of heel pain. J Am Podiatr Med Assoc 1997, 87:11-16.

78. Pfeffer G, Bacchetti P, Deland J, Lewis A, Anderson R, Davis W, Alvarez R, Brodsky J, Cooper P, Frey C, et al: Comparison of custom and prefabricated orthoses in the initial treatment of proximal plantar fasciitis. Foot Ankle Int 1999, 20:214-221.

79. Gross MT, Byers JM, Krafft JL, Lackey EJ, Melton KM: The impact of custom semirigid foot orthotics on pain and disability for individuals with plantar fasciitis. J Orthop Sports Phys Ther 2002, 32:149-157.

80. Woodburn J, Barker S, Helliwell PS: A randomized controlled trial of foot orthoses in rheumatoid arthritis. J Rheumatol 2002, 29:1377-1383.

81. Lin JL, Balbas J, Richardson EG: Results of non-surgical treatment of stage II posterior tibial tendon dysfunction: A 7- to 10-year followup. Foot Ankle Int 2008, 29:781-786.

82. Slattery $M$, Tinley $P$ : The efficacy of functional foot orthoses in the control of pain in ankle joint disintegration in hemophilia. J Am Podiatr Med Assoc 2001, 91:240-244.

83. Powell M, Seid M, Szer IS: Efficacy of custom foot orthotics in improving pain and functional status in children with juvenile idiopathic arthritis: a randomized trial. J Rheumatol 2005, 32:943-950

84. Novak P, Burger H, Tomsic M, Marincek C, Vidmar G: Influence of foot orthoses on plantar pressures, foot pain and walking ability of rheumatoid arthritis patients-a randomised controlled study. Disabil Rehabil 2009, 31:638-645

85. Clark H, Rome K, Atkinson I, Plant M, Dixon J: The clinical effectiveness of foot orthoses in rheumatoid arthritis. Rheumatology (Oxford) 2010, 49:Suppl. 171.

86. Cui Q, Milbrandt T, Millington S, Anderson M, Hurwitz S: Treatment of posttraumatic adhesive capsulitis of the ankle: a case series. Foot Ankle Int 2005, 26:602-606.

87. DiGiovanni BF, Nawoczenski DA, Malay DP, Graci PA, Williams TT, Wilding GE, Baumhauer JF: Plantar fascia-specific stretching exercise improves outcomes in patients with chronic plantar fasciitis. A prospective clinical trial with two-year follow-up. J Bone Joint Surg Am 2006, 88(8):1775-1781.

88. Kulig K, Lederhaus ES, Reischl S, Arya S, Bashford G: Effect of eccentric exercise program for early tibialis posterior tendinopathy. Foot Ankle Int 2009, 30:877-885.

89. Rompe JD, Cacchio A, Weil L Jr, Furia JP, Haist J, Reiners V, Schmitz C, Maffulli N: Plantar fascia-specific stretching versus radial shock-wave therapy as initial treatment of plantar fasciopathy. J Bone Joint Surg Am 2010, 92:2514-2522.

90. Williams $A E$, Bowden AP: Meeting the challenge for foot health in rheumatic diseases. The Foot 2004, 14:154-158.

91. Williams AE, O'Neil TW, Mercer S, Toro B, Nester CJ: Foot pathology in patients with Paget's disease of bone. J Am Podiatr Med Asoc 2006 96:226-231

92. Kamanli A, Suluhan O, Ozgocmen S, Kaya A, Ciftci I, Ardicoglu O: Measurement of Foot Bone Mineral Density in Rheumatoid arthritis: Its Application and Clinical Relevance. Turk J Rheumatol 2010, 25:56-62.

93. Kavlak Y, Demirtas N: Effect Of Foot Problems On Foot Function In Elderly Men. Turk J Geriatri 2010, 13:191-196. 
94. Goldstein CL, Schemitsch E, Bhandari M, Mathew G, Petrisor BA: Comparison of Different Outcome Instruments Following Foot and Ankle Trauma. Foot Ankle Int 2010, 31:1075-1080.

95. Rosenbaum D, Schmiegel A, Meermeier M, Gaubitz M: Plantar sensitivity, foot loading and walking pain in rheumatoid arthritis. Rheumatology (Oxford) 2006, 45:212-214.

96. Schmiegel A, Rosenbaum D, Schorat A, Hilker A, Gaubitz M: Assessment of foot impairment in rheumatoid arthritis patients by dynamic pedobarography. Gait Posture 2008, 27:110-114.

97. Trevethan R: Evaluation of two self-referent foot health instruments. Foot (Edinb) 2010, 20(4):101-108.

98. Martin RL, Irrgang JJ: A survey of self-reported outcome instruments for the foot and ankle. J Orthop Sports Phys Ther 2007, 37:72-84.

99. Landorf KB, Burns J: Health Outcome Assessment. In Merriman's Assessment of the Lower Limb. 3rd edition. Edited by Ben Y, Merriman LM. Philadelphia, PA 19103-2899: Churchill Livingstone, Elsevier Limited; 2009:33.

100. Button G, Pinney S: A meta-analysis of outcome rating scales in foot and ankle surgery: is there a valid, reliable, and responsive system? Foot Ankle Int 2004, 25:521-525.

101. Landorf KB, Radford JA: Minimal important difference: Values for the Foot Health Status Questionnaire, Foot function Index and Visual Analogue Scale. The Foot 2008, 18:15-19.

102. van der LM, Steultjens MP, Terwee CB, Rosenbaum D, Turner D, Woodburn J, Dekker J: A systematic review of instruments measuring foot function, foot pain, and foot-related disability in patients with rheumatoid arthritis. Arthritis Rheum 2008, 59:1257-1269.

103. Jannink MJ, deVries J, Stewart RE, Groothoff JW, Lankhorst GJ: Questionnaire for usability evaluation of orthopaedic shoes: construction and reliability in patients with degenerative disorders of the foot. J Rehabil Med 2004, 36:242-248.

104. Rogers JC, Irrgang JJ: Measures of Adult Lower Extremity Function. Arthritis Rheum 2003, 49:S67-S84.

doi:10.1186/1757-1146-6-5

Cite this article as: Budiman-Mak et al: A review of the foot function index and the foot function index - revised. Journal of Foot and Ankle Research 2013 6:5.

\section{Submit your next manuscript to BioMed Central and take full advantage of:}

- Convenient online submission

- Thorough peer review

- No space constraints or color figure charges

- Immediate publication on acceptance

- Inclusion in PubMed, CAS, Scopus and Google Scholar

- Research which is freely available for redistribution 